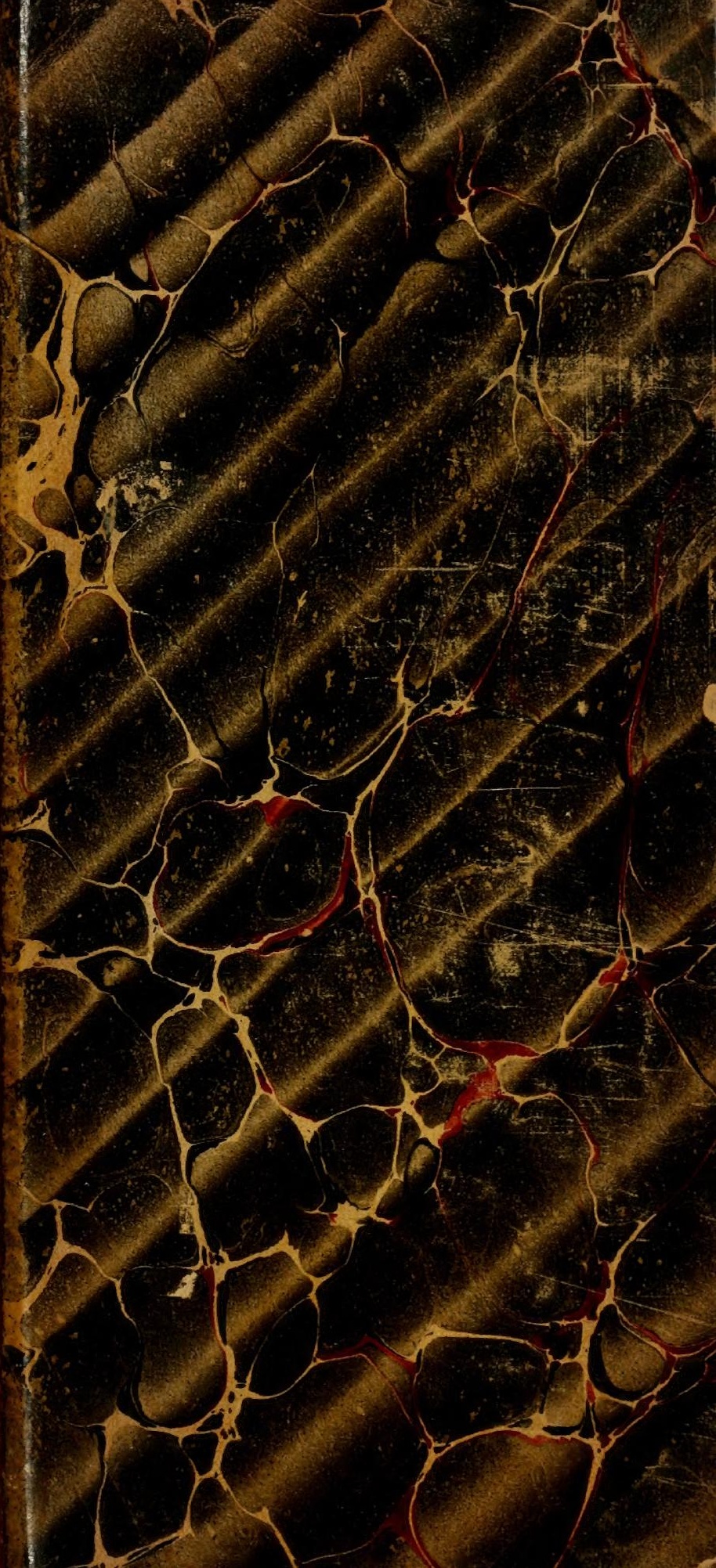




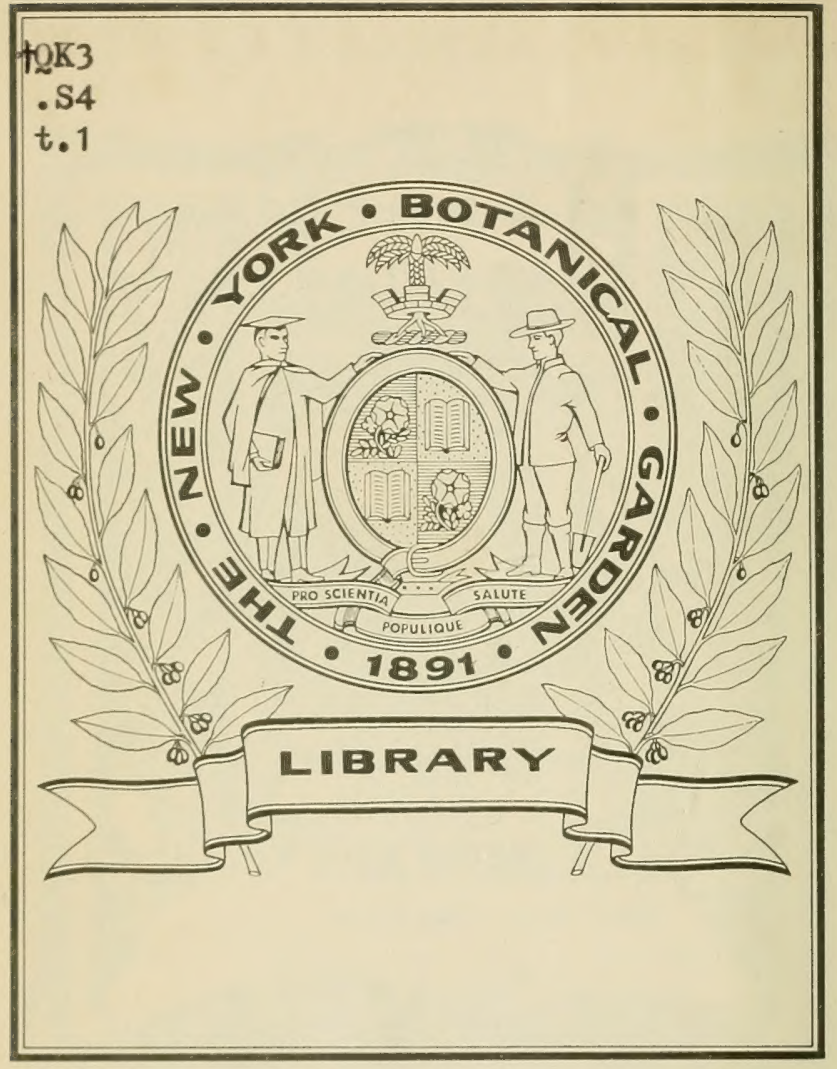




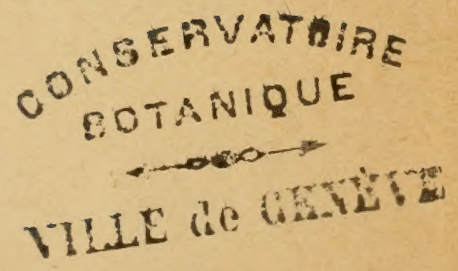





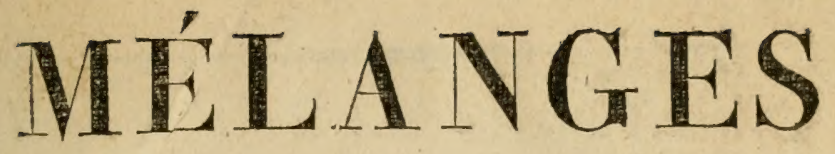

\section{BOTA IQUES}

0 U

\section{R E C U E I L}

D'OBSERVATIONS, MÉMOIRES, ET NOTICES SUR LA BOTANIQUE, P A $\mathbf{B}$

Nicolas Charles Seringe, Instituteur à l'Académie de Berne.

BHERARR

VOLUME I. DOTANICAL

Contenant une Critique de cinq décades de Roses desséchées et la Monographie des céréales de la Suisse.

BERNE, chez la Société Typographique. LEIPSIC, chez Ch. Cnobloch en commission.
1819 ,
CONSERVATOIAE
EOTANIQUE
VIIIE de GWHits 


$$
\begin{gathered}
+Q K 3 \\
.54 \\
V .1 \\
n 0.1-2
\end{gathered}
$$




\section{PRÉFACE.}

$\mathrm{D}_{\text {Ars ce 1. }}{ }^{\text {ex }}$ volume de mes Mélanges botaniques joffre $1 .{ }^{\circ}$ une partie des résultats de mes observations sur le genre Rosa; et $2^{\circ}$ la Monographie des céréales de la Suisse.

Quant aux Roses, je renvoye le lecteur au Musée helvétique, section botanique (*),

(*) Chaque livraison, de deux Nros., contient deux feuilles de texte in $4.0^{\circ}$, et deux planches coloriées; (au besoin on y joindra une 3ème feuille de texte, ou une 3ème planche.) Le prix de chaque livraison est de 3 francs de Suisse pour les souscripteurs, qui sont invités d'indiquer celle des deux éditions française ou allemande qu'ils désirent. On peut se procurer cha. que livraison séparément au prix de 3 fr. $12 \mathrm{~s}$. de Suisse, sans être obligé de s'engager à l'achat de tout l'ouvrage. - La première livraison est de 4 feuilles d'im. pression, et de deux planches, l'une colorjée et l'autre noire, elle contient $10^{\circ}$ Observations générales sur

I bes Roses; $2 .^{\circ}$ Description de la Rosa rubrifolia et 2 de ses variétés; $30^{\circ}$ Remarques sur les 6 premières co livraisons des Roses de Mr. Redouté. S'adresser à $D$ Mr. Burgdorfer, libraire à Berne, ou à l'Auteur. 
cahier $1 .^{\text {er }}$ et $2 .^{e}$, où se trouvent développées mes idées sur ce genre, que la culture et le désir de toujours faire de nouvelles espèces, ont rendu si difficile. La critique de mes Roses desséchées est en liaison avec ce premier travail : j'ai tâché de montrer quelques-uns des rapprochements, qui restent encore à faire dans les espèces des auteurs.

Je crois être parvenu dans ma Monographie des céréales de la Suisse à établir les espèces et les variétés avec plus de précision qu'auparavant. Ce mémoire est divisé en quatre parties : $\mathbf{1}^{\circ}$ Introduction à l'étude des céréales; page $69 ; 2{ }^{\circ}$ Description des genres, espèces et variétés des céréales, page 82 , 3. Maladies des céréales, p. $187 ; 4^{\circ}$ Usages économiques des céréales, $p$. 208. - La première partie est simplement destinée à l'explication des termes employés. Dans la seconde jai fait tous mes efforts pour établir les caractères et la synonymie. La troisieme traite des maladies des céréales. Dans l'impossibilité d'ajouter quelque chose au travail de Mr. DeCandolle, je me suis décidé à le transcrire; d'autant plus qu'il se trouve inséré dans deux ouvrages, qui ne sont pas entre les mains de tout le monde. En en donnant un extrait, 
j’aurais pu lui ôter de sa clarté, et comme je le crois très-propre à rectifier une foule d'idées fausses, répandues chez les agriculteurs, je n'ai pu résister à lui donner encore plus de publicité. J'ai réuni enfin dans la quatrième partie tout ce qu'il m'a été possible de me procurer sur les différentes productions des céréales.

J'ai rassemblé sous le titre de Herbarium cereale des exemplaires séchés des céréales et de leurs productions, qui pourront servir à vérifier les caractères que j’ai donné. Le catalogue s'en trouve à la fin de ce volume, pag. 228. J'ai terminé ce mémoire par des tables très-détaillées, au moyen desquelles on pourra facilement trouver les articles dont on aura besoin.

Je suis cependant loin de croire avoir amené à la perfection l'étude des céréales, et je recevrai avec la plus vive reconnaissance les observations que les botanistes et les agriculteurs voudront bien m'adresser; aidé de leurs remarques, des exemplaires murs, pressés ou en graines, que je les prie instamment de m'envoyer, je m'empresserai, en faisant les corrections que je croirai nécessaires, de leur en. témoigner toute ma gratitude. - Je dois aussi 
prévenir, que n'ayant eu pour le moment en vue, que la description des genres et des espèces des céréales suisses, il se pourrait fortbien, que les caractères que j’en ai donné, ne fussent pas assez exclusifs pour les distinguer d'autres plantes que je n'ai point étudiées, et dans ce cas j'ai encore l'espérance que les naturalistes voudront bien m'éclairer de leurs observations.

Berne, 20 Octobre 1818. 


\section{MELANGES BOTANIQUES,}

ou Recueil d'observations, mémoires et

$$
\begin{gathered}
\text { notices sur la botanique, }\left(^{*}\right) \\
\text { par N. C. SERINGE. }
\end{gathered}
$$$$
\text { N. } 1 .
$$

(Juillet 1818.)

Beavcoup de remarques à faire sur mes collections de plantes séches, auxqquelles il est impossible d'ajouter de grandes étiquettes, le désir de les rendre utiles, et bien des petites choses à noter, particulièrement sur les plantes de la Suisse, m’ont engagé à publier de temps en temps, sous le titre de Mélanges botanigues, le résultat de mes observations.

(*) Quinze feuilles in.8. formeront un volume, qui sera accompagné d'un titre et d'une table très.détaillée, afin de faciliter les recherches. $3 \mathrm{fr}$. de Suisse, ou $3 \mathrm{btz}$. (9 s. de France) par Nro. Afranchir lettres et argent. Chez l'auteur a Berne. 
Les collections de plantes séches ne remplisseni souvent pas le but d'utilité qu'on s'en était proposé; 1. $0^{\circ}$ parce qu'il est difficile d'avoir un nombre suffisant d'exemplaires semblables; $20^{\circ}$ que ces collections ne sont pas accompagncees de notes critiques; et $3 .^{\circ}$ qu'elles ne sont pas ordinairement disposées commodément pour être consultées. Ehrhart, élève de Linné, est un des botanistes qui a su rendre ses collections utiles; la précision qu'il y a mise, et le peu d'exemplaires qu'il en avait fait, les ont rendus très-rares. Les Messieurs Thomas ont fait connaitre beaucoup de plantes intéressantes, qu'ils ont recucillies dans lcurs nombreuses excursions; Mr. Schleicher, qui aurait pu rendre de grands services à la botanique, s'il avait cu en vue dans tous ses travaux le vrai but de la scicnce, s'est trop occupé de la partie lucrative. MM. Mougeot et Nestler ont publié, sous le titre de Stirpes Cryptogamae, ( 5 centuries) une très-belle collection de plantes cryptogames, qui est trèscommodément disposée pour être consultée; enfin M. Flörke a domé depuis peu quelques livraisons de Lichens, auxquelles il a joint des notes très-intéressantes, ct les amatcur's des Lichens attendent avec impaticnce la suite de cette belle collection. Je mettrai plus que jarnais un grand soin à ces herbiers; trop heureux si avec beaucoup de peine, de patience et de temps je puis offrir dans ces pelits travaux quelques résultats utiles.

Je me ferai un devoir de publicr aussi ce que mes correspondants voudront bien me communiquer de leurs remarques. Je tàcherai de donner à la 
fin de l'été un petit aperçu méthodique des Roses en présentant en mème temps les espèces et Ies variétés distribuées dans leurs groupes.

\author{
C R I T I Q U E \\ des Roses desséchées, (*) \\ de N. C. Seringe. \\ Par thateur luisméme.
}

J'Avars conçu depuis long-temps le projet d'étudier le beau, mais difficile genre des Roses; j'en publiai dès 1807 la $10^{\circ e}$ décade, la $20^{\circ}$ et $3 .^{\circ}$ parurent en 1508. Sans abandonner entièrement cette entreprise, $j$ 'ai continué à augmenter ma collection et à faire ça et là quelques remarques; mais enfin mon gout pour ce genre s'étant éveillé plus vivement l'année dernière, je me remis au travail, et,

(*) Mon principal but, en publiant mes décades de Roses, étant de faciliter ma correspondance, je les enverrả soit en échange de plantes ou d'ouvrages botaniques, soit au prix de $15 \mathrm{btz}$. ( $45 \mathrm{~s}$. de France); lettres et argent affianchis. Les exemplaires sont placés dans du papier blanc in-fol., et 5 décades seront toujours renfermés dans un carton en forme de livre,

$1^{*}$ 
tout en observant, je recueillis les matériaux des décades 4 et 5 , que j’offre aux amateur's de l'un de mes genres favoris. J'ai cru que mes remarques critiques sur mes Roses desséchées pourraient être de quelqu'intérêt, qu'elles engageraicnt quelques amateurs à me communiquer leurs observations, et que par là je pourrais concourir à lever les difficultés qui restent encore à vaincre, corriger les fautes que j'aurai pu commettre, et amener l'ćtude des espèces de ce genre au point d'en faire, conjointement avec mes collaborateurs, une monographie basée sur une longue observation.

Plusieurs incorrections dans la nomenclature, dans la synonymie, provenues du travail peu suivi et peu avancé, que j’avais fait sur ce genre, nécessitaient, pour que ma collection pat être de quelqu'utilité, que je retouchasse un peu ce travail, que je rétablisse les noms des auteurs primitifs, ct surtout que je rapportasse à ines excmplaires les synonymes des auteurs. Ayant fiit ces notes pour servir de texte à mes décades, $j$ 'ai été contraint de laisser les espèces dans l'ordre où je les ai publićes; mais à la fin l'on trouvera ces espèces, leurs variétés et leurs synonymes essentiels disposés alphabétiquement, afin d'abréger les recherches. 


\section{DE C A D I.}

1. ROSA ARVEISIS UNIFLORA GLABRA.

(R.procumbens Ser. Ros. dess. Déc. 1. n. ${ }^{\circ}$.)

R. arvensis Lin. Mant. 2. p. 245. - Willd. spec. 2. 2. p. 1066 n. 6. Baumz. ed. 2. p. 404. n. 31. - Hofim. Deutschl. Fl. ed. 1791. p. 175. n. 4. ed. 1500. p. 228. n. "3. - Sut. Fl. helv. 1.p. 299. n." 4. - Pers. Syn. 2. p. 47. n. 7. - Loisel. Fl. gall. 1. p. 294. n. ${ }^{\circ}$ 4. - Sinith Fl. brit. 2. p. 533. $n: 2$. -

R. foliis et rotundo fructu levibus, calicis foliis divisis, alba flore. Hall. Enum.p. 349. $n .^{\circ} 6$. - Hist. $n .^{\circ} 1102$.

R. SERPENS. Ehrh. Arb.35. (pedunculis scabris.)

R. REPENS Willd. Enum. 1. p. 547. n: 24. Baumz. ed. 2. p. 404. 'n. ${ }^{0}$ 32. (*) - Rau Enumh. Ros. p. 40. (avec la nombreuse synonymie qu'il $y$ éiablit.)

R. herponhodon Ehrh. Beitr. 2. p. 71.

(*) TVilldenow établit dans ce dermier de ses ouvrages sa $R$. repens sur la simple hispidité des pédoncules, qui sont lisses dans sa $R$. arvensis J'ai aussi quelquerois trouvé cette variété, mais plus rarement que la $R$. arvensis laevis, je l'ai vue encore l'année dernière sur le Bulpherg; elle a effectivement les pédoncules hérissés de glandes presquue sessiles, ce qui leur donne un aspect rude, mais $i$ est bien certain que ce n'est que la $R$. arvensis scabra. Ser. 
R. sempervirens prostrata Desi. Journ. bot. 1813. p. 113.

R. prostrata DeC. Cat. Monsp. p. 138, et Fl. fr. $n 0^{\bullet} 3714 . a$.

R. stylosa Desv. Journ. bot. 1809. 2. p. 317. et 1813. p. 113. T. 14. - DeC. Cat. Monsp. p. 138. $n 0^{\circ} 4$.

r Obs. Je n'ai plus aucun doute que ce ne soit à la $\boldsymbol{R}$. arvensis Lin. Mant., que se rapporte la synonymie que j'ai établie ci-dessus. Cette espèce a ses tiges toujours couchées quand quelque obstacle ne les force pas à se relever; les rameaux uniflores ou en corymbes de 3-8 fleurs, les pédoncules lisses, ou raboteux par les glandes presque sessiles, qui les couvrent quelquefois, les lanières du calice très-rarement pinnatifides, et qui ne dépassent pas le bouton avant son épanouissement, les styles réunis en une longue colonne glabre, et les fruits elliptiques et couleur de carmin à leur maturité, souvent terminés par la colonne des styles pertissante, mais non couronnés par les lanières calicinales caduques.

Rosier des champs, $R$. rampant, $R$. à longs styles. - All. Feld-Rose, hiriechende Rose. - L. Fréquent le long des lisières des bois.

\section{ROSA CINNAMOMEA.}

(R. cinnamornea Ser. Ros. dess. Déc. 1, n. ${ }^{0} 2$. )

R. cinnamomea L. Sp. 1.p. T03. n。 4. et Gaud, Fl. helv. manusc, 
R. COLLINCol Ehrh. Beitr. 2. p. 70. - Irall. fil. in Iiöm. arch. 1. fas. 2. p. 7. n. 26. 1. $\left({ }^{*}\right)$

Obs. On peut consulter la synonymie bien établie dans I'Enumera:io Rosarum de Mr. Rau, p, 52, et sa bonne description. D'ailleurs voyez la note que j'ai faite sur les Roses de Redouté, Musée helvétique, $\mathrm{n} .{ }^{\circ} 10$.

Rose canelle, Rose de Mai. --All. Zimmt-Rose - L. Rives et îles de l'Aar prés Berne, Lausanne au-dessus de Pully (Reyn.), Martigny et St. Branchier (Murith.)

\section{ROSA TOMENTOSA.}

(Ser. Ros. dess. Déc. 1. n. 3. Herb. port. cent. 5. $n 0^{\circ}$ 498.)

R. TOMIEntosa Smith Fl. brit. 2. p. 539. - De C. Fl. fr. 4. n. 3701 .

Obs. 1. Malgré toutes les tentatives quej'ai faites jusqu'à présent pour rapprocher cette espèce de la R.villosa et canina, avec lesquelles elle a beaucoup de rapports, je n’ai encore pu me décider à la réunir ni à l'une ni à l'autre; mais, avec M. Desvaux (Journal

$\left(^{*}\right)$ Mr. Gaudin avait aussi établi dans la Fl. helv. manuse. une $R$. versifolia fructibus globosis, pedunculisque laevibus, foliolis eilipticis dorso cinerascentibus, aculeis rectis, petiolis inermibus; mais ayant reconnu que ce n'était que la Rosa cinnamomea, il a détruit cette espèce. 
botanique 1813. p. 114. $R$. canina tomentosa) je crois que si on la réunit un jour à l'une des deux espèces, ce sera à la cunina à laquelle elle touche par la $R$. collina. Mes exemplaires récoltés près Berne (Egni) répondent parfaitement à la description laconique et lumineuse qu'en donne le Docteur Smith, Fl. brit. 2. p. 539. „Rosa praecedente ( $R$. villosa) omni"bus partibus minor est, et habitu cum $R$. canina "convenit, nisi quod folia undique pubescunt et sub " cinerea videntur. Rami erecto-patentes, sub́ fle"xuosi. Aculei caulini adunci, basi dilatati. Folio 3) Ia $5 \mathrm{vel} 7$, suaveolentia, elliptico-ovata, utrinque " mollissime tomentosa, serraturis glandulose ci"liatis. Petioli tomentosi, muricati. Pedunculi "terminales, subsolitarii, setis rigidis glandulo"sis muricati. Germen etiam muricatum ellipti" cum. Calyx glanduloso-hispidus. Petala basi „ albida, superne pulcherrime rosea. Fructus ova"tus, coccineus, muricatus magnitudine et forma "R. caninae proximus."

Obs. 2. C'est à la $R$. iomeniosa Smith que j'ai rapporté Ia Rosa n.1105, Hall. Hist. , mais elle appartient à la R. villosa $\mathrm{L}$.

Obs. 3. D'après les exemplaires qu'a eu la complaisance de m'envoyer le Professeur Rau, et d'après la description qu'il donne (Enum. Ros, p. 154.) de sa R. villosa mollissima, je n'ai aucun doute qu'clle n'appartienne à la R. tomentosa de mes Roses desséchées; (quoique ses excmplaires soient en tout un peu moins grands que les miens); conséquemment 
la R. mollissima Gmel. Fi, bad. 2. p. L09., dont j’ai confronté la description avec mes exemplaires, ainsi que la $R$. villosa mollissima Willd. Sp. 2. p. 1070 , et probablement aussi les autres citations de Willd. Prod. n. ${ }^{\circ} 1237$, et $R$. dubia Fl. Verth, p. 263. que je ne connais que par ce qu'en dit Mr. Rau, s'y rapporteront probablement aussi. - Voyez encore n. ${ }^{0} 39$ de mes Roses desséchées, la $R$. tomentosa lanceolata.

Fr. Rosier drapé. R. cotonneux. - Angl. Downyleaved Dog-rose. - L. Rare aux environs de Berne (Egni, Belpberg), mais plus fréquent, à ce qu'il parait, dans les expositions chaudes du Jura.

\section{ROSA MOSCHATA SEMI-PLENA.}

(R. moschata Ser. Ros. des. Déc. 1. n.0 4.)

R. Moschata Ait. kew. 2. p. 207. d'apre's Willd. Spec. 2. 2. p. 1074. - DeC. Fl. fr. 4. n. ${ }^{0} 3715$. Cat. Monsp. p. 138. $n .{ }^{0} 3$.

R. OpSOSTEMma Plenum Ehrh. Beitr. 2. $p$. 72 .

R. moschata flore semi-Pleno (") Red. Ros. Livr. 7. p. 99. (37. superbe fig.)

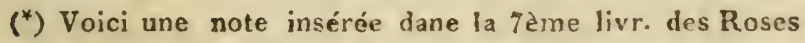
de Mr. Redouté, qui pourra peut.être intéresser.

n'il est vrai, ainsi que l'ont dit les Botanistes et les voyageurs, que ce Rosier soit naturel au sol de l'Indostan, on doit croire que les fleurs, qui y répandent dans toute leur intensité, celle oc̉eur de musc particulière à l'espèce, sont employées, avec les Roses du Kachmyr, si renommées dans l'Orient par leur beauté 
Obs.1. Cette espèce est remarquable par ses corymbes ordinairement très-fournis de petites fleurs blanches, ses styles saillants, libres, velus; ses pédoncules articulés vers le tiers inférieur et velus; ses lanières calicinales longues, dépassant le bouton, et se défléchissant ávant l'épanouissement total de la fleur et enfin par ses stipules très-étroites et acérées.

et leur parfum, à la composition de l'essence précieuse que les Indiens nomment $A^{\prime} t h e r$, et qui n'est antre chose que l'huile essentielle de Rose, quı surnage audessus de l'eau-de_rose distillée, qu'on ramasse, tandis que cette eau est encore chaude, par le moyen d'un peu de coton attaché au bout d'une baguette.

2) L'histoire de la découverte du procédé aujourd'hui employé pour obtenir cette essence est aussi curieuse que singulière. M. Langlès, dans un petit ouvrage qu'il a publié, intitulé : Recherches sur la decouverte de l'essense de rose. (Paris 1804 in-12. 47 pages) a communiqué à ce sujet un document précieux tiré de l'histoire générale du Mogol, le voici : Dans une fête. donnée par la princesse NouraDyhan à l'empereur Djihanguyr, cette courtisanne poussa le luxe et la richesse, jusqu'à faire circuler dans les jardins un petit canal rempli d'eau-de-rose. Tandis que l'empereur se promenait avec elle sur les bords du canal, ils apperçurent une espèce de mousse qui s'était formée sur l'eau, et qui nageait à sa surface. On attendit pour la retirer qu'elle fut au bord, et on reconnut alors que c'était une substance de Rose que le soleil avait recuite, et, pour ainsi dire, en masse. Tout le sérail s'accorda à reconnaître cette substance huilcuse pour le parfum le plus délicat que l'on connut dans l'Inde. Dans la suite l'art tâcha d'imiter ce qui avait été d'abord le produit du hasard et de la nature." - 
Obs. 2. Mr. Redouté (Roses Livr. 1.p. 33) a figuré la variété à fleurs simples, il en réprésente le fruit trèspetit, obovoïde -alongé, et couronné par une houpe de longs styles velus. Voyez d'ailleurs Mus. helv. Rem. sur les Ros. de Mr. Redouté, n. 05 .

Rosier musqué à fleurs semi-doubles, la musquée à fleurs semi-doubles. - All. Bisam-Rose. - L. Jardins, on la dit originaire de Barbarie.

\section{ROSA INDICA SIMPERFLORENS SEMI-}

\section{PLENA.}

(R. semperflorens Ser. Ros. dess. Déc. 1, n.0 5.)

R. Semperflorens Willd. Spec. 2. 2.p. 1078 n.0 34. (non DeCand.)

Obs. Outre ce que j'ai noté dans le Mus. helv. à. I'article : Remarques sur les Roses de Mr. Redouté, n. ${ }^{0}$ 13. 14. 15. 19. 21., j'ai oru remarquer que cette espèce a un caractère assez constant, c'est que chaque pédicelle est plus gros que l'extrêmité du xameau ou pédoncule qui le porte.

\section{ROSA ALPINA VULGARIS.}

(R. alpina Ser. Ros. dess. Déc. 1. n. ${ }^{0} 6$. - Herb̆. portat. cent. 5. n.0 495.)

R. alpina Lin. Spec. 1.p. 703, n.0 1. - Willd. Spec. 2. 2. p. 1075. n. ${ }^{0}$ 26. - DeC. Fl. fr. n. ${ }^{0} 3712$. var. a. - Sturm Deutschl. Fl. 26. Heft (bien médiocre) - Sut. Fl. helv. 1. p. 301. $n_{0} 0^{0} 12$. 
R. inermis Mill. Dict. n.o 6. (d'après Willd.)

R. RUPEsTris Crantz. Aust.p. 32. n.0 6. (en excluant les deux derniers synonymes.)

Ros a non spinosa, calycis foliis indivisis, fructu o7longo. Hall. Enum. p.348. n. $0^{0}$ 2. - Rosa inermis, foliis septenis, glabris, 'calycis segmentis indivisis. Hall. Hist. n. ${ }^{0} 1107$.

R. alpiNa vulgaris Gaud. Fl. helv. ined. n.014.

Mon exemplaire est parfaitement caractérisé par

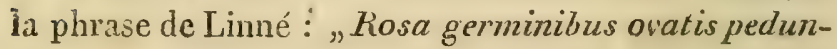
"culis sub hispidis, caule inermi."

Obs. 1. M. DeCand. a déja réuni (Fl. 4.n. ${ }^{0} 3712$ et 5. n.0 3712. a p. 536.) presque toutes les variétés de la $\boldsymbol{R}$. alpina, pyrenaica, lagenaria etc. J'ai plusieurs fois trouvé la preuve de la nécessité de cette réunion dans les courses que j'ai faites; j'ai vu, non seulement sur le même individu, mais sur le mêrme rameau, un pédoncule lisse ainsi que le fiuit et l'autre, quand les fleurs sont géminées, ce qui est plus rare, le pédoncule et le fruit hérissés de poils glandulcux, ou quelquefois l'ovaire hérissé d'un côté, et lisse de l'autre. J'espère que quand les Botanistes auront étudié les autres espèces, comme Mr. DeCandolle l'a fait de la $R$. alpina, ils ne répugneront nullement à réunir des espèces qu'ils croyaient d'abord très-distinctes.

Obs. 2. Je serais aussi fort peu surpris quon accuit bientot la certitude que la $R$. incrmis Delaunay, (Bon-jardinier, annće 1808. p. 717. n. ${ }^{0} 24$.) qui figure dans quelques jardins sous le nom de 
R. sinensis prcecox imermis, n'est qu'une variété à fleur's roses et doubles de la $R$. alpina; mème époque de floraison, même forme de feuilles. L'épaisseur des folioles de cette variété, leur glaucescence raproche cncore cette $R$. inermis (R. alpina muliiplex Ser.) de la $R$. alpina latifolia Ser. Remarques sur les Roses de Mr. Redouté. Mus. helv. n. ${ }^{0}$ 17. et figurée dans les Roses de Fiedouté, Livr. 3. pag. 57. sous le nom de $R$. alpina pendulina ( $R$. pendulina Ait.) Ce rapprochement répugnera d'autant plus les Botanistes qu'ils observeront cette $R$. inermis fortement taillée, car alors les feuilles acquérent de beaucoup plus grandes dimentions, et la plante est alors prodigieusement dénaturée, mais quand on observe des individus non taillés, les feuilles sont beaucoup plus petites, et ressemblent à celles de $12 R$. alpina. J'en ai vu chez Mr. Steltler, un buisson entièrement abandonné à la nature, qui était énorme et de la plus grande beauté, il fleurissait à la fin de Mai; il avait quelquies cent boutons ou fleurs épanonies dun effet vraiment admirable. Celte variété a ses fieurs, qui ne s'épanonissent qu’à moitié, d'un rose très-pur, ce qui fera peut-être encore rejeter par quelques personnes le rapprochement que je propose, mais la $R$. a!jina offre des passages du cerise-foncé au rose bleuâtre et peut bien se transformer aussi en rose-pur; ce dont on trouverait bien facilement d'autres exemples dans les Roses et dans d'autres fleur's. 
7. ROSA RUBIGINOSA VULGARIS.

(R. rubiginosa Ros. dess. Déc. 1. n. 0 7.)

R. Rubiginosa Lin. Mant. 2.p. 564. - DeC. Fl. fr. 4. n.0 3710. - Smith Fl. brit. 2.p. 540. n. 5. Sut. Fl. helv. 1. p. 298. n. 2.

R. Folirs subtus RUbiginosis et odoratis Hall. Enum. p. 350. $n .{ }^{0} 7$. - R. SPINIS ADUNCIS, FolLIS subtus rubiginosis Hall. hist. n.01103. (en excluant la R. Flava Lobel, qui est la R. EgLANTERia $L$.)

R. RUbiginosa vUlgaris Willd. Enum.1.p. 546. 7.0 18. a. - Rau Enum Ros. p. 130 - Willd. Baumz. ed. 2. p.397. n.0 22. a. - Red. Ros. Livr. 6. p. 93.

Obs. 1. La $R$. rubiginosa Schl. Cat. de 1815, envoyée en 1817, a sur le même exemplaire trois rameaux de fleurs; l'un, uniflore, a le tube de son calicehispide, le second biflore, et le troisième triflore; le tube calicinal dans ces deux derniers est presque lisse. - Sa $R$. rubiginosa resinosa a les rameaux uniflores, le pédoncule presque nul, les fruits lisses et les folioles très-arrondies.

Obs. 2. La R. agrestis Savi, d'après l'exemplaire que m'a envoyé Mr. Schleicher, qui dit l'avoir déterminé sur un exemplaire de Mr. Savi même, répond bien à la $R$. rubiginosa triflora, Willd. Enum. 1. p. 546. n. 0 18. B., par l'alongement des rameaux, la grandeur des folioles et ses pédoncules 1.2. ou 3. flores, hispides, ainsi que par son calice, et 
ses aizuillons petits et faibles. -- D'ailleurs l'exemplaire que m'a donné Mr. DeCandolle, de la $R$. agrestis Savi, lequel lui avait été envoyé par Mr. Bertoloni, qui communique avec $\mathrm{Mr}$. Savi, appartient bien à la $R$. rubiginosa, mais il est uniflore et a le pédoncule et le tube du calice hispide.

Obs. 3. La $R$. umbellata Leers., Gmel. et Rau (d'après DeC. Fl. fr. 5. n. ${ }^{0} 3695$ a. p. 532), dont le Professeur DeCandolle m'a aussi donné un exemplaire, appartient bien certainement à la $R$. rubiginosa et formera ma $R$. rubiginosa tenuiglandulosa ( $R$. tenuiglandulosa Mérat Fl. par 189, d'après Mr. DeCandolle.) Elle ressemble beaucoup à la $R$. rubiginosa triflora figurée par Redouté Ros. Livr. 6. p. 93. (34.) L'exemplaire est triflore, le calice lisse, les pédoncules peu hispides et les feuilles garnies de très-petites glandes odorantes.

Rosier rouille, Eglantier sauvage, Eglantier rouge, Rosier à odeur de pomme-reinette. - All. Wein-rose. - Angl. Siveetbriar, Eglantine. - Terrains arides.

\section{ROSA RUBRIFOLIA LAEVIS.}

(Ser. Mus, helv. R. rubrifolia Ser. Ros. dess. Déc. 1. $n, 0$ 8.)

\section{R. RUBRIFOLIA Will.}

Voyez Musée helv. pour la synonymie.

Fr. Rosier à feuilles rouges, variété lisse. - I. Scheideck. 


\section{ROSA CANINA.}

(R. canina Ser. Ros. Dec. 1. n.0 9.)

R. Canina Lin. Spec. 1.p. 704. n.0 10. Hall. Hist. n.0 1101. a.

R. Canina $a$. DeC. Fl. fr. n. 03716.

R. canina glabra Desv. Joutr. bot.1313, p.114. n. ${ }^{0}$ 5. $a,\left(^{*}\right)$

Obs. Cette espèce est une de celles qui, par l'importance que l'on a mise à l'absence ou à la présence des poils lymphatiques sur les pétioles, sur la face inférieure des folioles, ou même sur leur face supéricure, par l'absence ou la présence des poils slanduleux sur les pédoncules et le tube du calice a foumi le plus d'espèces.

On est

$\left(^{*}\right)$ Le travail de Mr. Desvaux, Journal botanique 1813 p. 104, (quoique reniermant de grandes vérités) ayant été présenté trop laconiquement, a paru fort étrange à un grand nombre de naturalistes; il est sans aucune critique rajsonnée des espèces et des variétés et conséquemment ue pouvait guère ramener les esprits sur ce que je crois cependant approcher beaucoup de la vérité. Ainsi que bealicoup d'autres personnes, je n'ai pas rendu au travail de ce naturaliste, sur les Roses, toute la justice qu'il mérite : il faut long.temps étu. dier les individus vivants avant de pouvoir le regarder comme le résultat de longues et pénibles recherches. Sans avoir d'abord consulté le travail de Mr. Desvaux, sans aroir été entraîné par son opinion, que je n’appréciais pas, nous arriverons je crois souvent a peu près aux mêmes résultats; ce qui doit, ce me semble, donner à nos recherches une certaine confince, ou aus moins engager les Botanistes d ne pas les rejeter sane examen. 
Obs. 2. On est donc convenu, d'appeler R. ca* nina une Rose, dont les styles sont courts; les pédoncules lisses; le fruit ovoïde, lisse, couleur de carmin à sa maturité; les lanières pinnatifides; et dont les folioles, souvent simplement, mais aiguement serretées, sont dépourvues de poils lymphatiques et de poils glanduleux. Mais ce n'est là que l'état le plus commun de l'espèce, voyez dans cette collection les $\mathrm{N} .^{\circ} 35$. $R$. canina collina, et 36. $R$. canina nitens., qui ne sont bien certainement que quelques-uns des différents états de la même espèce.

\section{ROSA RUBIGINOSA SEPIUM.}

(R. sepium Ser. Ros. dess. Déc. 1. n. ${ }^{0}$ 10. - Herb. port. cent. 5: $n .0496$.

R. sepium Thuill. (*) Fl. par. ed. 2.p. 252. Desv. Journ. bot. 1813. p. 116. var. alba. - DeC. Fl.fr. 5 p. 538. n. $0^{0}$ 3716. a. - Schl. Cat. 1815.

R. Myrtifolia Hall. fil. Herb.

R. Canina b. sepiuni DeC. Fl. fr. 5. $n .03716$.

R. RUbiginosa Glabra Rau Enum. Ros. p. 138. (d'après ses propres exemplaires à fieur's rouges, et d'aprés sa description.)

R. Sepiuni в. Gaud. Fl. helv, manuse. n, ${ }^{0} 11$.

R. RUBiginosa B. Gaud. Fl. helv. manusc. $n,{ }^{0} 12$ :

(*) R. elatior; ramis undique aculeis recurvis armatis: foliolis plerumque septenis, parvulis, ovatis, acutis, subtus uti petiolus glandulis conspersis : fructibus oh. longo-ovatis pedonculisque glabris. 
Obs. 1. Je suis bien certain par un exemplaire de Mr. Thuillier même, par ceux de l'herbier du Professeur Decandolle, par beaucoup d'autres, reçus de divers points de la Suisse, de la France et de l'Allemagne, que la $R$. sepium Thuill. est la mème que l'exemplaire n. ${ }^{0} 10$ de mes Roses desséchées, mais je ne crois pas qu'on puisse la séparer de la R.rubiginosa L. Voyez mes notes sur ces deux espèces des auteurs, Mus. helv., Rem.sur lẹs Roses de Mr. Redouté, n. ${ }^{0} 38$.

Obs. 2. Mr. Gaudin, dans sa Flora helvetica manuscrite, établit deux varićtés de la $R$. sepium ( $R$. fructibus pedunculisque hispidis, à laquelle il cite la $R$. agrestis Schl., qui lui aura peut-être envoyé un autre exemplaire qu'à moi, ct $\boldsymbol{R}$. sepium ambigua ? Desv. Journal bot. 1813. p. 117, mais qui ne se rapporte pas à cette variété. - B. fructibus pedunculisque glaberrimis. Mais l'exemplaire que m'a envoyé Mr. Schleicher, et qu'il dit avoir été confronté avec celui qu'il tient de Mr. Savi, et espèce, dont je ne conmais pas la description, est plus voisin de la $R$. rubiginosa que de la $R$. sepium des auteurs. Cet exemplaire de Mr. Schlcicher est Ie point de contact des dcux espèces des auteur's, car sur le même rameau se trouvent des folioles lancéolées et d'autres ovées. - Je ferai connaître par la suite plusicurs jolies variations de cette $R$. rubiginosa sepium. 


\section{DE G A D II.}

\section{ROSA EGLANTERIA LUTEA.}

(R. eglanteria Ser. Ros. dess. Déc. 2. n.011.)

R. egganteria L. Spec. 1.p. 703. - DeC. Fl. fr. 4. n. ${ }^{0} 3694$. var, $a$.

R. Chlorophylla unicolor Ehrh. Beitr. 2.p. 69 et 70. $n .08$.

R. Lutea Ait. kew. 2. p. 200.n.01. Willld. Spec. 2. 2. p. 1064 .

R. Cerea Rossig. Ros. T. 2. (d'aprés plusicurs auteurs.)

R. FOETIDA All. Fl. pedem.n. ${ }^{0} 1792$.

Rosa Hall. Hist. n. ${ }^{9} 1103$ (confondue avec la R. rubiginosa. L.).

Obs. La patrie de cette Rose est entièrement inconnue; elle fait l'ornement des bosquets par ses belles fleurs simples et jaunes. - Mr. Redouté a parfaitement figuré cette variété dans son magnifique ouvrage sur les Roses. Livr. 4. p. 69. - Mr. Gaudin a cité cette variété de la $R$. eglanteria dans sa Flora helvetica, manuscrite, seulement sur la foi de Haller, mais personne ne l'a retrouvée depuis Mr. de Coppets, qui parait l'avoir vue dans des haies près de Lassara, où elle aura probablement été transportée d'un jardin. Voyez encore Musée helv., Remarques sur les Roses de Redouté n. ${ }^{0} 23$.

Fr. Rosier églantier, églantier jaune. - Alt. Gelbe Rose.

$$
2 \text { * }
$$


12. ROSA EGLANTERIA BICOLOR.

(R. eglanteria bicolor Ser. Ros. dess. Déc. 2. n. 12.)

R. BICOLOR Jacq. Hort vitud. 1. p. 1. T, 1. $n .03$.

R. PUnice Rossig T. 5. (DeCand.)

R. eglanteria bicolor DeC. Fl. fr. 4. 72.03694 .

R. eglantetia punicea Red. Ros. Livr. 4.p.71. (très-belle.)

Cette variété de la $R$. eglanteria est cultivée dans presque tous les jardins; elle n'a bien certainement de rapports avec la $R$. rubiginosa, avec laquelle quelques auteurs l'ont confondue, que par l'odeur de ses feuilles. - Voyez Mius. helv. Iiem. sur les Roses de Redouté, n. ${ }^{0}$ 24.)

Fr. Rose capucine, Rose comtesse.

13. ROSA SULPIIUREA MULTIPLEX.

(R. sulphurea floire pleno Ser. Ros. dess. Dée.2. n. ${ }^{0}$ 13.)

R. lutea mutitiplex Bauh. Pin. 483.

R. sulphurea Ait. Hort hew. p. 201. - DeC. Fl. fr. 4. n.0 3695. - Red. Ros. Livr. 1.p. 29. (trés-belle.) - Willd. Spec. 2. 2.p. 1065.

R. Glaucophylda Ehrh. Beitr. 2. p. 69.

Voyez Mus. hel. Rem. sur les Ros. de Redoul's, n. 03.

Fr. Rose jaune-soufre. - All. Schwefelgelbe Rose. - L. l'Orient (d'aprés Willdenow.) 
14. ROSA CAROLINA IIISPIDA.

(R. carolina Ser. Ros. dess. Déc. 2. n.0 14.).

R. carolina Lin. Spec. 1. p. T03. - Tilld. Spec. 2. 2. p. 1069, n.011. - Baumz. ed. 1. p. 311., ed. 2. p. 388. $71 .{ }^{0} 11$.

R. Corymbosa Ehrh. Beitr. 4.p. 21. n. 13.

R. virginiana Duroi Baumz. 1. p. 353. (d'aprés Ehrh. et Willd.)

R. Carolina corymbosa Red. Ros. Livr. 5.p. 81. ( $n .{ }^{0}$ 28. feuilles figurées plus lar'ges que dans mes exemplaires.)

Les variétés de celte espèce, qui n'est pas d'une grande apparence, mais qui a l'avantage d'orner long-temps dans l'arrière saison les bosquets de ses flcur's couleur cerise, ont quelquefois sur le même buisson des rameaux uniflores et des corymbes multiflores, des calices lisses (ce qui constitue ma variété $R$ caroïina laevis.) - D'ailleurs la $R$. carolina est bien certainement distincte de la $R$. cinnamomea, qui a pour caractère d'espèce des folioles minces, flasques et écartées, des stipules larges et obtuses et des fleurs printanieres; tandis que la $R$. carolina a des folioles fermes, coriaces et rapprochées, des stipules élroites et aigues et des fleurs tardives.

Fr. Rosier de la Caroline. - All. carolinische Rose. - L. Amérique septentrionale.

15. ROSA CENTIFOLIA muscosa multiplex.

(R. muscosa flore pleno Ser. Ros. dess. Déc. 2. n. 015. 
R. muscosa Willd. Spec. 2. 2.p. 1074. n.022.DeC. Fl. fr. 4. n.0 3705 .

R. muscosa multiplex Red. Ros. Livr. 2.p. 41. $n .09$.

Obs. Voyez les différences entre la $R$. centifolia et ses variétés et la $R$. calendarum Gmel. et ses variétés, Ser. Mus. helv., Rem. sur les Roses de Redouté ( $R$. centifolia, n. 1. 7. 8. 9. 21.26. 27. et R. calendarum, ou bifera $n .0$ 1. 13. 20.

Fr. Rose à cent feuilles mousseuse double. All. Moos-Rose.

16. ROSA CENTIFOLIA MULTIPLEX RUBRA.

(R. centifolia More pleno Ser. Ros. dess. Déc. 2. $\left.n .{ }^{0} 16.\right)$

R. Centifolia Lin. Spec. 1. p. 704. n.0 7. DeC. Fl. fr. n.0 3704. var. a. - Willd. Spec. 2.2. p. 1071. n. $0^{0} 15$.

R. centifolia multiplex Red. Ros. Livr. 1. p. 25. (1.)

Obs. Cette espèce se reconnait à ses styles courts, aux lanières de son calice ascendantes avant l'entier épanouissement de la fleur, à ses boutons courts ovoïdes, à ses fruits globuleux ou ovoïdes? à ses folioles molles, flasques et garnies de larges serretures. - Voyez d'aillcurs Mus. helv. Rem. sur les Roses de Redouté $n .^{\circ} 1$. et pour d'autres variétés $\mathbf{n}^{\circ} 7$. 8.9. 21. 26. 27 . 
$F_{r}$. Rose à cent feuilles double et rose. - All. Garten-Rose. - L. Originaire de Perse, d'après Mr. Biberstein.

17. ROSA CALENDARUM MULTIPleX ROSEA.

R. calendarum flore pleno rosea Ser. Ros. dess. Déc. 2. n. ${ }^{0}$ 17.)

R. omntum calendarum Ross. Ros. T. C. (d'après $M r$. DeCand.)

R. semperflorens Desf. Cat. 175. (non Curt.)DeC. Fl. fr. 4. $n .03706$.

R. Calendarum Gmel. Fl. bad. 2.p. 430.

Obs. Cette espèce se distingue toujour's des variétés de la $R$. centifolia par les lanières de son calice déféchies dès le commencement de la floraison, ses boutons longuement ovoïdes, ses fruits ovoïdes alongés; ses folioles moins flasques que dans la $R$. centifolia, souvent plus arrondies, et ses serretures plus petites. - Voyez d'ailleurs MIus. helv. Rem. sur les Roses de Redouté $n .^{\circ} 1$. 13. 20. - Il serait bien possible que la $R$. dainascena subalba. Red. Ros. Livi. 4. p. 63. ne fut que le type de cette espèce.

Fr. Rosier de tous les mois, Rosier des quatre saisons, Bouquet tout fait, Rosier de deux fois l'an. 
18. ROSA CALENDARUM MULTIPLEX ALBA.

(R. calendarum flore albo pleno Ser. Ros. dess. Déc. 2. n.0 18.)

Obs. Variété à fleurs blanches et à pédoncules courts et roides du Rosier de tous les mois. Quand cette espèce ne croìt pas dans un terrain très-meuble, elle a des pousses peu vigoureuses, des pédoncules courts et roides et des aiguillons très-forts et très-nombreux entre les trois ou quatre feuilles supérieures.

Fr. Rosier de tous les mois à fleurs blanches.

19. ROSA CENTIFOLIA POMPONIA MAJOR.

( $R$. pomponia major flore pleno Ser. Ros. dess. Déc. 2. $n .{ }^{0}$ 19.)

R. PROVincialis $a$. IVilld. Spec. 2. 2. p. 1071.

R. pomponia DeC. Herb. (non Fl. fr. n. ${ }^{0} 3707$.)

Obs. Je suis plus que jamais persuadé que ce n'est qu'une variété de la $R$. centifolia. - C'est un exemplaire parfạitement semblable à celui du n. ${ }^{\circ} 19$ de mes Roses desséchées, qui se trouvait en 1817 dans l'herbier du Professeur DeCandolle sous le nom de R. pomponia.

Fr. Rose a cent feuilles gros pompon, gros pompon rose. 
20. ROSA CENTTIOLIA POMPONIA MINOR.

(R. pomponia minor flore pleno Ser. Ros. dess. Déc. 2. $n{ }^{0}{ }^{0}$ 20.)

R. Provincialis B. Willd. Spec. 2. 2. p. 1071. $n .014$.

R. POMPONIA DeC.Fl.fr. 4.n. ${ }^{0} 3707$. (non Herb.) R. burgundica Pers. Syn. 2. p. 48, n. ${ }^{0} 18$.

Obs. 1. Voyez d'ailleurs Mus. helv. Rem. sur les Roses de Red. n.. 21. - Il y a toujours dans toutes les variétés de la $R$. centifolia une certaine tendance des feuilles à se flétriv, à cause du tissu faible et mince de leurs folioles; celui de la $R$. calendarum offre plus de fermeté, et enfin les folioles de la $R$. gallica sont manifestement coriaces.

Obs. 2. Mr. Redouté a figuré (Roses Livr. 4. p. 65. n. 21 ) l'état intermédiaire de grandeur entre la $R$. centifolia pomponia major et la $R$. centifolia pomponia minor, état que j’ai nommé $\mathrm{R}$. centifulia pomponia media (Mus. helv. Rem. Ros. $10^{\circ} 21$.)

Obs. 3. J'ai encore vu, au jardin botanique de Berne, sous le nom de $R$. poinponia kingston portugal, une autre variété de la $R$. centifolia pomponia media (R. pomponia Ried. Ros. Livr. 4. p. 65.) jointe aux feuilles de la $R$. centifolia pomponia major. Voici, ce me semble, les différents passages de la $R$. centifolia, en commençant par les plus petites variétés. 
1. R. Centifolia pomponia minor.

(R. pomponia Ser. Ros. dess. Déc. 2. n. 20.)

2. R. CENTIFOLIA POMPONIA MEDIA.

(R. pomponica Red. Ros. Livr. 4. p. 65., fleurs plus grandes que celles de la $R$. pomponia Ser. Ros. dess. n. ${ }^{\circ} 20$. avec la grandeur des feuilles de ce même exemplaire.)

3. R. CENTIFOLIA KINGSTON-PORTUGal.

(Fleurs de la R. pomponia Red. Ros. Livr. 4. p. 65., avec le feuillage de la variété suivante.)

$4 .^{\circ} R$. Centifolíta mintata.

(R. miniata Hort. Bern., dont les feur's sont plus petites que celles de la $R$. centifolia pomponia major, avec les feuilles de la variété suivante )

5. R. CENTIFOLIA POMPONIA MAJOR.

(Fleurs plus volumineuses que celles de la variation 4. et feuilles presqu'aussi grandes que dans la Rose à cent feuilies ordinaire des jardins R. centifolia.)

$6 .^{\circ} \mathrm{R}$. Centifolia multiplex.

(Red. Ros. Livr. 1. p. 25. n. 1. (R. centifolia.) Puis suivent les nombreuses variations à grosses fleurs de la $R$. centifolia et muscosa des Auteurs.)

Fr. Petit rosier pompon. 


\section{D É C A D E III.}

\section{ROSA GALLICA REMENSIS VIOLACE.}

(R. remensis Ser. Ros. dess, Déc. 3. n. ${ }^{0} 21$.

R. parvifolia Ehrh. Beitr. 6. p. 97. - Willd. Spec. 2. 2. p. 1078. Enum. 1. p. 547. n. ${ }^{0}$ 25., Baumz. ed. 2. p. 405. n. ${ }^{0} 33$.

R. REMENSIS DeC. Fl. fr. 4. $72 .{ }^{0} 3708$.

R. burgundica Gmel. Fl. bad. 2,p. 431.

Obs. 1. Plus j'examine cette espèce, et plus je suis persuadé que ce n'est qu'une variété de la $R$. gallica; même époque de floraison, même compacité, même sécheresse des feuilles, même glaucescence de leur face inférieure, même forme de boutons et de lanières calicinales, presque toujours entières, et qui ne dépassent pas les pétales lorsque la fleur n'est qu'en bouton. Folioles toujours trèsrapprochées, très-sessiles, rudes; stipules étroites.

Obs. 2. Je possède des exemplaires qui ont à la base du rameau des folioles entièrement rondes, tandis que celles de la seconde ou troisième feuille au-dessus sont lancéolé-aigues.

Obs. 3. Mr. DeCandolle indique que ce Rosicr est commun sur les montagnes des environs de Dijon, je désirerais bien en avoir des exemplaires récoltés sur les lieux, ct surtout savoir si on n'cn trouve pas des individus à fleurs simples, violettes, roses ou blanches. 
Obs. 4. Voyez Musée helv. Rem. sur les Roses de Mr. Redouté, n. 21 . et 26.

Fr. Rosier de Meaux, R. de Rheims, R. de Champagne, $R$. d petites feuilles.

\section{ROSA GALLICA MULTIPLEX.}

(R. gallica flore pleno Ser. Ros. dess. Déc. 3. n. $\left.{ }^{0} 22.\right)$

R. RUBRA, Flore SEMI-PLENo Bauh. Hist. 2. p. 34 .

R. Gallica a. Lin. Spec. 1. p. 704. - DeC. Fl. fr. 4. n. ${ }^{0} 3719$. - Willd. Spec. 2. 2.p.1071.n. ${ }^{0} 16$.

R. alsstriaca Crantz, Aust.p. 86.

Obs. C'est l'espèce dont on a un nombre de variétés vraiment effrayant. Voyez Musée helv. Rem. sur les Roses de Redouté, n. ${ }^{\circ} 21.25 .26$.

Fr. Rosier de France, Rosier de Provins. All. Zucker-Rose.

23. ROSA GALLICA VERSICOLOR.

(R. gallica variegata flore pleno Ser. Ros. dess. Déc. 3. $n .{ }^{0}$ 23.)

R. Gallica b. versicolor Lin. Spec.p. 704. DeC.Fl.fr.4.n.0 3709. - Willd. Spec. 2. 2.p. 1072.

Obs. 1. C'est encore à cette variété, d'aprìs Mr. Redouté Ros. Livr. 5 p. 76. B. B. que l'on doit rapporter les synonymies suivans. $R$. holosericen, Lob. 2. ico 207. - R. praenestina var, plena. Mill. Dict. T. 221. fig. 2. - R. beloica carnea rubro 
striata. Ross. Beschr. 1. p. 66. - R. gallica variegata vel. Rosa mundi. Andr. Ros. fig.

Obs. 2. Cette variété, très-répandue dans les jardins, est presque toujours demi-double, elle a ses pétales $10^{\circ}$ ou d'un blanc rosé, panachés de bandes rose-foncées, ce qui a fait alors domner à cette variété le nom de Rose ceillet, mais qu'il ne faut pas confondre avec la $R$. centifolia unguiculata; 2. ${ }^{\circ}$ ou bien le fond est rose et les bandes violettes ou pourpres, et alors les fleuristes la nomment Rose zharbrée, $\left(R_{+}\right.$gallica versicolor marmorea.)

$F r$ Rose de Provins aillet, $R$. ceillet, R. miparvie, $R$. bigarée, Rose-monde.

\section{ROSA GALLICA holosericE..}

(R. Holosericea Ser. Ros. dess. Déc. 3. n.0 24.),

Obs. Cette Rose que, malgré ses lanières calicinales fortement pinnatifides ct scs folioles presque rondes, je rapporte encore, mais avec doute, à la R. gallica, n'est pas la même variété, que celle, à laquclle appartient la pumila Jacq, dont elle est cependant très-voisine. (Voyez n.。 32 et 33. de mes Roses desséchées et de cette Critique.) En conséquence il faut effacer la synonymie que $\mathrm{j}$ 'ai rapportée sur mon étiquette.

Fr. Rose veloutée, Rose cramoisi-veloutér, $R$. de velours. 
25. ROSA TURBINATA MULTIPLEX.

(R. turbinata flore pleno. Ser. Ros. dess. Déc. 3. n. $\left.{ }^{0} 25.\right)$

R. turbinata Ait. kew. 2. p. 206. - DeC. Fl. fr. 4. n. ${ }^{0}$ 3703. - Willd. Spec. 2. 2. p. 1073. n.0 20.

R. caitipanulata Ehrh. Beilr. 6. p. 97.

R. rrancofurtensis Desf. Cat. 175.

Obs. Cette singulière cspèce, dont les fleurs d'un violet sale, s'épanouissent toujour's assez mal, est peu recherchée des fleuristes; ses calices sont trèsremarquables par leur évasion campaniforme, due au développement des pétales, qui le dilatent. Ses folioles, presque circulaires ont leur's nervures trèsdroites et ressemblent assez, dans l'état frais, aux feuilles du charme (Carpinus betulus), par leur gaufrure.

Fr. Rosier en toupie, R. de Francfort, R. à gros cul, R. campanule. - All. kreiselförmige Rose.

\section{ROSA ALPINA IATIFOLIA.}

(R. pedulina Ser. Ros. dess. Déc. 3. n.0 26.)

R. SANGUISORBAE MAJORIS FOLIO, FRUCTU-LONGO pendulo Dill. elth. 325. T. 245. f. 31T. (d'aprés Lin. et Willd.)

R. pendula Lin. Spec. 1. p. 705. 7.012.

R. pendulina Ait. kew. 2. p. 208. - Willd. Spec. 2. 2. p. 10个6. n.0 28 . 
R. alpina pendulina Red. Ros. Livr. 3. p. 57. (en excluant tous less synonymes cités.)

Obs. J'ai déjà dit, Musée helv. Riem. sur les Roses de Redouté, n. ${ }^{0} 17$., que je ne croyais pas que la $R$. pendulina Ait. ent encore été trouvce en Europe. Mr. Redouté l'a dessinée sur un individu cultivé, mais il ne l'a pas vue spontanée. Cette variété, à gros fruits, a, comme toutes les autres variétés de la $R$. alpina, son pédoncule défléchi après le floraison, mais un peu plus court dans mes exemplaires que dans la figure citće de Mr. Redouté, et le tube calicinal, ainsi que le pédoncule, ou muni de poils glanduleux sur une ou sur toutes ses parties, ou en est totalement privé. Souvent l'un des pédoncules du même rameau cst lisse et l'autre hérissé. En un mot l'hispidité dans cette variété est extrêmement inconstante. - Voyez d'ailleurs Musée helv. Rem. sur les fioses de Redouté, n. ${ }^{0} 17$.

Fr. Rosier des Alpes à larges feuilles. $-L$. Amérique septentrionale. Cultivé dans les jardins paysagistes à cause de ses fleurs précoces et de ses beaux fruits carminés.

27. ROSA INDICA CIINENSIS SEMI-PLEN.1.

(R. chinensis flore pleno Ser. Ros. dess. Déc. 3. ת. ${ }^{0}$ 27.)

R. CHINENSis Willd. Spec. 2.2.p. 1078. n. ${ }^{0} 35$.

R. Indica vulgaris Red. Ros. Livr. 3. p. 51. (superbe figure.) 
Obs. 1. Les fleurs de cette variété sont surtout extrêmement jolies au printemps, ćpoque où les pétales se développent lentement et très-bien, tandis qu'en été l'épanouissement est trop prompt et les fleurs sont alors moins grandes et moins agréables à l'œil.

Obs. 2. "Ce Rosier est aujourd'hui devenu populaire dans l'Europe entière. Il est le plus répandu des arbustes de ce genre. On le cultive dans tous les jardins : on le rencontre dans les palais et dans les chaumières. Il a été introduit en Europe par les Anglais, qui l'ont apporté de l'Inde, et il a fleuri pour la première fois en Angleterre, vers l'année 1793 , dans le jardin de Mr. Parson de Rickmannsworth, dans le Hertfordshire. Ses fleurs, d'une grande fraicheur, sont légèrement odorantes, sur-tout au moment de leur épanouissement. On le multiplie très-aisément de boutures qui, lorsqu'elles sont faites au mois de Février, donnent souvent des fleur's dans la même anncé. On l'a traité long-temps comme une plante d'orangerie, mais aujourd hui on le cultive en pleine-terre, et il a bravé jusqu'à présent les hivers les plus rigoureux." Red. Ros. Livr. 3. pag. 51. - Voyez Musée helv. Rent. sur Jes Ros. de Red. no ${ }^{\circ}$ 13. 15. 19. 21.

Fr. Rosier du Bengale, R. des Inules, R. de la Chine. - All. chinesische Rose. - Angl. Pale China Trose - I. In Inde. 


\section{ROSA FRAXINIFOLIA.}

(R. fraxinifolia Ser. Ros, dess. Déc. 3. n.02S.)

R. FRAXINifolia Gmel. Fl. bad. 2.p. 413.

R. Branda Ait. kew. 2.p. 202. (diaprès Willd. Spec. 2.2. p. 1065. n. 0.4.)

R. alpina laevis Red. Ros. Livr. 3.p. 59. n.018. (excellente figure.)

Obs. 1. Malgré toutes les tentatives que $j$ ai faites jusqu'à présent pour réunir cette espèce à la $R$. cinnamomea; je n'ai pu leur trouver des rapports que de groupe, mais jamais je n'aurais eu l'idée de la joindre à la $R$. alpina, dont elle est curtainement très-distincte. Voyez Mus. helv. Rem, sur les Roses de Mr. Redouté, $\mathrm{n} .{ }^{\circ} 18$

Obs.2. La nécessité de prouver ce que j'ai avancé, et de fixer les idées sur cette intéressante espèce m'a décidé à transcrire l'excellente description que Mr. Gmelin a cionnée (Fl. badensis 2.p.413.) de sa $R$. FRAXINIFolis : "Germinibus globosis pedunculisque glabris, foliolis eliptico-lanceolatis (obovatolanceolatis) serratis utrinque giabris, petiolis glaliris aculeolatis, caule subinermi. - Frutex 5-6 pedalis. Rami praelongi, erecíi, virides, saepe pubescentes, lucidi subinermes. Folia alterna. petioTata, impari-pinnata, majuscula. Foliola breviter pedicellata, opposita, trium parium, elliptico-lanceolata (obovato-lanceoi.) aqualiter argute serrata, utrinque glabra, supra saturate viridia, sub- 
tus pallidiora, impari longius pedicellato. Petioli glabri, aculeolis rarioribus quandoque proditi. Stipulae lato-lanceolatce, serrulatae, glandulosate. Flores caulem ramosque terminantes, subsolitarii. Pedunculi glaberrimi, uniflori. Germen globosum, glabrum. Calycinae laciniae quinque, caudatosubulatae, integrae, apice foliaceae, villoso_sericae. Corolla, petala quinque subemarginata, palIide rubella, calyce breviora. Styli brevissimi, pilosi. Baccae maturae globosae, glabra, rubrae, demum nigrae, splendentes.

Fr. Rosier à feuilles de Frêne. - All. Eschenblättrige hose, Hudsonsbay-Rose. - L. Amérique, Baie de Hudson, d'où elle a été transportée dans nos jardins paysagistes, ou elle se propage, traçant beaucoup. Les jeunes individus sont hérissés vers le bas d'aiguillons fins, semblables à ceux des jeuzzes frambroisiers, (Rubus idaeus) tout comme dans la R. alpina, mais ils disparaissent bientòt.

\section{ROSA CINNAMOMEA MULTIPLEX.}

(R. cinnamornea flore pleno Ser. Tios. dess. Déc. 3. $\boldsymbol{n}^{0} 29$.

R. cinnomomea majalis Red. Ros. Livr. 7. p. 105. (41. bonne figure.)

Obs. 1. C'est la variété à fleurs doubles de la R. cinnamomea L. (Ser. Ros. dess. n. ${ }^{\circ}$. et Mél. 1. p. 6.) - Mr. Redouté a figuré une très-belle branche de cette variété, dont deux rameaux sont uni- 
flores, et le troisième biflore, tandis qu'en Suisse les fleurs sont plus souvent disposées en corymbes de 3-6 fleurs; les rameaux sont aussi réprésentés très-effilés, ce que je n'ai jamais vu.

Obs. 2. Les jeunes pousses de cette variété présentent, comme l'a aussi remarqué Mr. Redouté, une efflorescence qui se retrouve rarement dans les individus spontanés. Les pétales, plus foncés que dans la plante à fleurs simples, sont d'un rose vineux.

Fr. Rosier canelle à fleurs doubles. - L. Jardins.

30. ROSA ALBA multtplex.

(R. alba flore pleno Ser. Ros. dess, Déc. 3. n. ${ }^{0}$ 30.)

R. ALBA Lin. Spec. 1. p. 705, $\pi .013$.

R. alba B. Willd. Spec. 2. 2.p. 10s0. n.0 39. DeC. Fl. fr. 4, n. ${ }^{0} 371 \%$.

Obs. Cette espèce, qui présente aussi un assez grand nombre de variétés, est extrêmement répandue dans les jardins; elle est toujour's reconnaissable à son feuillage glaucescent, à ses folioles arrondies, à ses lanières calicinales toujour's profondément pinnatifides et à une odeur particulière indeffinissable et que j'ai cru retrouver dans toutes les variétés. Voyez encore le $\mathrm{n}^{\circ}$ - suivant.

$\mathrm{Fr}$. Rosier blanc à fleurs doubles, - All. weisse Rose. - L. Jardins. 


\section{D É C A D E IV.}

31. ROSA ALBA SEMI-PLENA.

(Ser. Ros. dess. Déc. 4. n.0 31.)

Obs. 1. Variété mi-double de la R. alba Lin.Cette espèce, que j’ai toujours vue à pétioles tomenteux, à folioles suborbiculaires et poilues en dessous, à pédoncule et calice hispides, présentera bien certainement aussi, quand on l'observera très-soigneusement, des variétés sans poils lymphatiques et sans poils glanduleux.

Obs. 2. Il me semble aussi que cette Rose se distingue de quelques autres espèces par la longueur de la partie libre de ses stipules aigues.

Fr. Variété à fleurs semi-doubles de la Rose blanche. - L. Jardins.

\section{ROSA GALLICA INAPERTA.}

(Ser. Ros. dess. Déc. 4. n. ${ }^{0} 32$.

Obs. 1. Cette plante, que je rapporte avec un peu d'incertitude à la gallica, est étiquetée au Jardinbotanique de Berne, R. Vilmorina, Rose transparente de Vilmorin, nom qui rappellerait, je crois, la R. centifolia curnea, Red. Ros. Livr. 5. p. 79. (27.) ce qui est impossible, car la plante, sur laquelle mes excmplaires ont èté récoltés, me semble devoir être rapportée à la $R$. gallica, par ses folioles très-sessiles, 
épaisses et coriaces, ses stipules étroites et ses longs styles.

Obs. 2. J'ai été un moment tenté de la regarder comme une variété de la $R$. calendarum Gmel. à cause de son tube calicinal alongé, et de ses lanières défléchies avant l'épanouissement total de la fleur; mais je penche plutôt pour le premier rapprochement. Il serait mème possible que ce fut une variation de la $R$. gallica agatha, Red. Livr. 5. p. 76. (sans fig.) - J'implore particulièrement sur cette plante l'aide des Botanistes qui s'occupent des Roses.

Fr. Rosier de Provins mi-clos.

\section{ROSA GALLICA PUMILA.}

(Ser. Ros. dess. Déc. 4. $12 .{ }^{0} 33$.

R. SYlvestris RUbella Parvo Frutice $J$. Bauh. Hist. 2. p. 35. (fig. passabl.)

R. pUMita panNonica flore rubello J. Bauh. Fiist. 2. p. 35. (sans fig.)

R. pUMu. Clus. Hist.p. 117. (sans fig.) - Jacq. Fl. Austr. 2. p. 59. T. 198. - Hoffm. Deutschl. Fl. (1800) p. 229.n.0 \%. - Willd. Spec. 2. 2.p. 1072. zz.0 19. - Sut. Fl. helv. 1. p. 301. n.0 10. - Sturm Deutschl. Fl. 34 Heft. (copie diminuée et bien médiocre de la figure de Jacq.) - Waht Fl. caucas. p. 150 n.0 491. (d'apris Rau.) - Thom. exs. Schl. Cat. 1815. (le grand exempl. cult.)

Rosa Hall. Enum.p. 348. n.0 3. - Hist.n.01104. et Tom. 3. in add.p. 188. 
R. CHAMAERHODON Will.? ? Hist. 3.p. 555. n.013. (Tous les synonymes de Haller, de J.Bauh. et de Rai. Hist. 1469. 6. mais non la description.)

R. Gallica pumila DeC. Fl.fr. 4. $n 0^{0} 3709$. Gaud. Fl. helv. manusc. n. ${ }^{0}$ 8. - Red. Ros. Livr. 5. p. 76 .

Obs. 1. Cette variété, dont les exemplaires m'ont été envoyés de Genève par Mr. Girod, ressemble, quoique un peu plus en petit, à la $R$. pumila Jacq. Ceux que j'ai reçus du midi de la France, de la Bohême etc. se ressemblent tous. C'est certainement de cette souche que seront sorties les innombrables variétés de la $R$. gallica, et directement la R. gallica holosericea, $\mathrm{n} .{ }^{0} 24$ de mes Roses des. séchées.

Obs. 2. J'ai trouvé dans quelques exemplaires. les styles de cette Rose saillants, et d'autres dépassent à peine le bourrelet où s'insèrent les ćtamines, de manière que dans cette espèce, il ne faut pas mettre à la longueur des styles une trop grande importance. En général dans la plupart des variétés de la $R$. gallica cultivées les styles sons trèssaillants: $\left(^{*}\right)$

Obs. 3. Cetle variété se distingue à ses aiguillons fins et nombreux, dont la base seule souvent existe, ce qui rend les rameaux très-rudes au toucher, (caractère commun à presque toutes les varié-

(*) Je donnerai bieniôt quelque chose sur les mons. truosités des Roses. 
tés ) à ses folioles très-sessiles, presque circulaires (plus rarement un peu alongées) très-coriaces, (caractère propre à l'espèce) et qui paraissent annoncer de la tendance à persister toute l'année dans les climats chauds, ou en serre. Les stipules sont étroites, (comme dans toute l'espèce) et les lanières calicinales tendent à se diviser.

Obs. 4. L'exempl. de la $R$. pumila, que Mr. Rau m'a envoyé, a ses folioles moins rapprochées que celui de mes Roses desséchées. plus lancéolées, moins sessiles; et appartient certainement à la $R$. gallica; ce sera de cette souche que seront sorties les $\boldsymbol{R}$. gallica à folioles alongées.

Fr. Rosier de Provins nain. - All. niedrige Rose. - L. France, Suisse, Bohême, Italie.

\section{ROSA GALLICA HYBRIDA.}

(Ser. Ros. dess. Déc. 4. n. ${ }^{0}$ 34.)

R. Gallica hybrida Gaud. (caule altiori, rami elongati, styli longiusculi, infra villosis, foliolis glabris, laciniis calycinis minus dissectis. Gaud. Fl. helv. manusc. $n .{ }^{0}$ 8.)

R. HYbrida Schl.Pl. helv.cent.1.n.0 54. Cat. 1815. Koch et Ziz. Catal. plant. p. 10. et 21. n. ${ }^{0} 17$.

R. Gemnata Rau Enum. Ros. 95. et Herb.

Obs. Mes exemplaires de $R$. gallica hybrida resscmblent parfaitement, mais en plus grand, ̀̀ celui que j'ai de la $R$. hiybrida de Mr. Schlcicher, 
et à ceux de la $R$. geminata Rau; ils ont été récoltés sur un individu très-rigoureux, qui n'est jamais taillé, et qui est cultivé au Jardin-botanique de Berne sous le nom de $R$. montana. Les fleurs en sont blanches, grandes, légèrement lavées de rose au moment de l'épanouissement, teinte qui disparait aussitot, et qui devient plus intense au premier moment de la dessication. Les folioles ont cette consistance coriace et cette glaucescence de la face inférieure, qui se retrouve dans toutes les variétés de la R. gallica; mais comme l'individu est planté dans un terrain humide et froid, il est en tout plus grand que les excmplaires de Mr. Schleicher. Quoique à fleurs simples, il n'a jamais porté de fruits, probablement à cause de sa position défavorable.

Fr. Rosier de France hybride, L. cultive.

\section{ROSA CANINA COLLINA.}

(Ser. Ros. dess. Déc. 4. n. ${ }^{0}$ 35.)

R. Collina Jacq. Fl. austr. 2, p. 58. T. 197.Sturm Deutschl. Fl. 34. Heft. (copie diminuée et médiocre de la fig. citée de Jacq.) - DeC. Fl. p. 4. n.0 ${ }^{3702 .}$ - Murith. Guid. p. 91. - Willd.Sp.2. 2. p. 1078. n. ${ }^{0}$ 32. - Schl, Cat. 1815.

R. Canina Colitna Desu. Journ. bot. 1813.p. 115.

R. dumetonum Thiill. Fl. par. ed. 2. p. 250. n. ${ }^{0}$ 2. DeC. Fl. fr. p. 534.n. ${ }^{0}$ 369s. a. Schl. Cat. 1815.

R. Canina Dumetorum Desv. Journ. bol. 1813. p. 115. DeC. Fq. fr. 4. n. 3716 . \% 
R. corymbifera Borkhaus. Koch et Ziz. Cat. 1514 n.0 19. -- Gmel. Fl. bad. 2. p. 424. n. 764.

R. puberula Desv. ined. (Herb. DeC.)

R. Borkinusi Gaud. Fl. helv. manusc. n. ${ }^{0}$ 10. $\left({ }^{*}\right)$

Obs. 1. J'ai acquis l'intime persuasion, par l'étude sur le frais, par l'examen des Roses de l'herbier de Mr. DeCandolle, qui m'a confié avec le plus grand désintéressement sa nombreuse collection, parcelles de l'herbier de Mr. Gaudin, par les exemplaires

(*) Mr. le Pasteur Gaudin, avec lequel j'ai eu le plaisir de converser, l'année dernière, une journée sur les Roses, Saules, Saxifrages, Aconits, a bien voulu me permettre de copier l'article Rosa de sa Flora helvetica et mia confié trois exemplaires, sur lesquels il a étahli trois des quatre variétés de sa $R$. Borkhausii, dont voici les caractères : Rosa fructibus ovatis pedunnculisque glabris, aculeis subulatis curvulis, petiolis foliolisque ovatis tomentosis. C'est sur J'exemplaire de la $R$. collina Schl, cent. n. 48 . que Mr. Gaudin a fait sa var. A., et il est parfaitement conforme à ceux de ma $R$. canina collina n. ${ }^{0} 35$. - La var. B. $R$. fructibus globosis, folinlis subrotundis obtusioris bus, a été établie sur un exempl. récolté au Simplon, et que je crois devoir rapporter à la $R$. tomentosa Smith; le duvet qui en couvre les folioles est court et trèsmol. - La var. C. a pour caractère : $R$. fructibus ovatis, foliolis ellipticis vel lanceolatis, laciniis ca. lycinis integriusculis. $R$. collina $B . D e C$.? - Enfin la var. D. R. pubescens foliolis undique prrecipue dorso pubescentibus, sub simpliciter serratis est la $R$. pu= bescens Schl. Cat. 1515, et me semble encore appartenir à la $R$. canina collina, mais les folioles sont moins larges que celles de mes exemplaires. 
séchés que m’a envoyés Mr. Schleicher, par la lecture attentive de la description de la $R$. collina Jacq. par la confrontation de la figure, qu'il en a donncé, avec les exemplaires de mes Roses desséchées, $n .^{\circ} 35$. que cette $R$. collina doit être rapportée à la $R$. cani$n a$, et que cet état n'est dû qu'à la compacité et à la sécheresse du terrain. Toutes les parties n'ont pu prendre leur développement comme lorsque la plante croit dans un endroit moins sec ou ombragé.

Obs. 2. La face inférieure des folioles, dans cette variété, est velue sur les nervures et conséquemment aussi sur le pétiole. Les rameaux sont les plus grands quej'aie pu récolter sur les individus qui sont près de Berne, les autres sont généralement très courts, et les branches entières ressemblent parfaitement à la figure citée de Jacquin.

Obs. 3. Les exemplaires de la $R$. collina, que je dois à la générosité de Mr. Rau, appartiennent probablement aussi à la $R$. canina, mais non à ma variété collina. Les folioles sont moins rapprochées, peu velues, les pétioles moins tomenteux et plus hispides, les pédoncules, les calices irrégulièrement hispides et plus alongés que dans la $R$. collina Jacq. , et les aiguillons droits et minces.

Fr. Rosier des chiens, variété des collines. All. Hügel-Rose. - L. collines séches des environs de Berne. 
36. ROSA CANINA NITENS.

(Ser. Ros. dess, Déc. 4. n.0 36.)

R. NITENS Hall. fil. Herb.

R. Canina nitens Desv. Journ. bot. 1813.p. 114 .

Obs. Cette variété, 1, 2, rarement 3-flore, est remarquable par son feuillage d'un verd lustré, comme celui de la $R$. lucida. Les deux faces dẹ ses feuilles, minces et très simplement serretées, entièrement glabres; les pétioles légèrement tomenteux, ainsi que la nervure médiaire; les pédoncules et le calice sont entièrement lisses et la fleur d'un rose pâle et presque inodore.

$F_{r}$. Rosier des chiens à feuilles luisantes. $-L$. Belplerg.

\section{ROSA TOMENTOSA GLABRIUSCULA.}

(Ser. Ros. dess. Déc. 4. n. ${ }^{0}$ 37.)

R. canina pilosiuscula? Desv. Journ. bot. 1513. p. 115.

Obs. Je ne puis considérer mes exemplaires que comme une variété de la $R$. tomentosa Smith, dont les folioles sont moins tomenteuses que dans les variétés $110^{\circ} 3$. et 38. de mes Roses desséchées. Les pédoncules et les calices sont hispides et les pétioles tomenteux et glanduleux.

Fr. Rosier tomenteux glabriuscule, - L. Belpberg. 
38. ROSA TOMENTOSA IANCEOLATA.

(Ser. Ros. dess. Déc. 4. n. ${ }^{0}$ 38.)

Obs. Folioles un peu plus alongées que dans la variété n. ${ }^{0} 3$. de mes Ros. dess.; d'ailleurs mêmes caractères, feuilles tomenteuses sur les deux faces, pétioles tomenteux et hispides, fleur's d'un rose trèspâle comme dans les deux autres variétés.

Fr. Rosier tomentersx à feuilles lancéolées. L. cult.

39. ROSA INDICA HUMILIS.

(Ser. Ros. des. Déc, 4, n.0 39.)

Obs. 1. Tous les caractères en miniature de la R. indica. J'ai trouvé sur le même individu des feuilles et des fleurs semblables à celles de cet exemplaire, et quelques feuilles beaucoup plus grandes.

Obs. 2. Cette variété a toujours une certaine tendance à avoir l'extrêmité libre de ses pétales acuminée à la manière de la $R$. indica acuminata Red. Ros. Livr. 3. p. 53. (15), mais elle tient plutot à la souche de la $R$. chinensis Willd. qu'à celle de la $R$. semperflorens Willd. Voyez les autres variétés n. 5 et 27 . de mes Ros. dess. et Musée helv. Rem. sur les Ros. de Red. n. ${ }^{\circ}$ 13. 14. 15. 19. 21.

Fr. Rosier nain du Bengale. - L. Cultivé en orangerie, mais pas plus délicat que les autres variétés. 
40. ROSA RUBIGINOSA MAJOR.

(Ser. Ros. dess. Déc. 4. no 40.)

R. RUbiginosa major Red. Ros. Livr. 6.p. 94. (Tableau, sans figure.)

Obs. Cette fort belle variété, à corymbes de 4 à 8 fleurs, d'un beau rouge cerise et demi doubles, est très-élevée et remarquable par ses très-grandes feuilles luisantes presque orbiculaires. Pédoncule et calice hispides.

Fr. Grand églantier sauvage à fleurs mi-doubles. - L. cult., Jard. bot. de Berne.

\section{D ÉCA D E V.}

\section{ROSA LUCIDA.}

(Ser. Ros. Déc. 5. n.0 4i.)

R. IUCIDA Ehrh. Beitr. 4. p. 22. n.014. (*) Willd. Spec. 2. 2. p. 1068. n.010. Enum. 1.p. 544 n. 0 7. Baumz. ed. 1. p. 310., ed. 2. p. 387. n. ${ }^{0} 10$. - Pers. Syn. 2. p. 48. n.0 12. - Red. Ros. Livr. 2. p. 45. - (11 fig. mal colorée.) - Ser. Mus. helv. Rem. Ros. n.0 11.

(*) Rosa foliis pinnatis : foliolis ovato-lanceolatis, obtusiusculis, grosso-serratis, glaberrimis, nitidis : petio. lis subaculeatis, glabris; corymbis paucifloris; pedun. culis subhispidis; germinibus depresso-globosis, sub. hispidis; foliolis calycinis integris.

Ehrh. 
Obs. Ce Rosier, un peu tardif et du groupe des crinnamomées $\left({ }^{*}\right)$ a des folioles coriaces, luisantes, lisses, elliptiques, simplement et obtusément dentées; ses larges stipules atteignent toujours la première paire de folioles; le tube de son calice, globuleux-déprimé, est ordinairement hérissé, ainsi que les pédoncules, et les lanières calicinales sont appendiculées à leur sommet.

Rosier à feuilles luisantes. - Glänzende Rose. - L. Amérique boréale; réussit très - bien en pleineterre.

\section{ROSA PIMPINELLIFOLIA MACROPHYLA.}

(Ser. Ros. dess. Déc. 5. n. 42.

Obs. Ce grand développement des rameaux, et sur-tout des feuilles, n'est dû qu'à un terrain humide et meuble. - Voyez Musée helv. Rem. Ros. $11 .{ }^{\circ} 29$. (R. pimpinellifolia mariaburgensis. Red. Ros. Livi. 5. p. 48.) et n. ${ }^{0} 30$, (R. pimpinellifolia pumila Red. Ros. Livr. 5. p. 85.) les raisons qui m'ont engagé à adopter le nom de $R$. pimpinellifolia, de laquelle la $R$. spinosissima ne diffère pas.

Fr. Trariété à grandes feuilles du Rosier pimprenelle à fleurs blanches. - L. Cult.

(*) Voyez (Musće helv.) le tableau des groupes des Rosiers, par Mr. DeCandolle. 


\section{ROSA PIMPINELLIFOLIA MYRIACANTHA.}

\section{(Ser. Ros. dess. Déc. 5. n. ${ }^{0}$ 43.)}

R. myriacantha DeC. Fl.fr. 4. n.0 3698. et 5 . p. 533.

Obs. 1. Arbuste nain; rameaux courts et conséquemment plus garni d'aiguillons, pédoncules hérissés:

Obs. 2. J'ai vu les exemplaires de la $R$. myriacantha (DcC. Herb.), j'en ai reçu de Montpellier par Mr. Requien, je dois encore à Mr. DeCandolle des exemplaires récoltés sur les individus que ce savant naturaliste a fait transporter dans le Jardin de Montpellier; leurs folioles circulaires ont un peu grandi , leurs aiguillons sont beaucoup moins nombreux, (les jets étant plus alongés) beaucoup moins fins; et je suis très-persuadé que si cette $R$. myriacantha était transportée dans un pays humide et froid, elle prendrait peu-à-peu entièrement la forme de notre $R$. spinosissima suisse, dont les folioles sont souvent ovales, beaucoup plus grandes, les pédoncules et le tube du calice ordinairement lisses et les fleurs blanches.

Obs. 3. Mr. Buchwalder m'a envoyé, de Delémont, des exemplaires de $R$. spinosissima L., dont les folioles variènt beaucoup sur le mème rameau, et dont quelques pédoncules (de la même branche) sont lisses en tout ou en partie, d'autres hérissés. Les folioles sont aussi beaucoup moins gran- 
des que celles des exemplaires que Mr. Girod m'a envoyés de Genève.

Obs. 4. Quant à moi je suis certain que les $R$. spinosissima L. Spec. 1. p. 705. n. ${ }^{\circ} 14 .-R$. pimpinellifolia. L. Spec. 1. 703. n. ${ }^{\circ} 2 .-R$. myriacantha. DeC. Fl. fr. 4. n. 3698 et 5. p. 633. - R. altaica. Willd. Enum. 1. p. 543. n. $0^{\circ}$. (R. spinosissima. Pallas et Fischer, Herb. DeCand.), et très-probablement $R$. scotica Desf., n'appartiennent qu'à la même espèce.

Fr. Rosier pimprenelle trés-épineux. $-L$. Montpellier.

44. ROSA PIMPINELLIFOLIA SEMI-PLENA ALBA.

(Ser. Ros. dess. Déc. 5. n.0 44.

R. Spinosissima nàna flore albo mult. Andr. fig. (Red, Ros. Livr. 5. p. 84.)

Obs. Cette jolie variété à fleurs demi-d oubles, greffée au Jardin-botanique de Berne sur une haute tige, forme une boule, couverte de charmantes fleurs. Ses pédoncules sont lisses, son calice évasé à sa gorge par le grand nombre de styles libres, qui saillent au milieu de la fleur, et en partie par 10 à 12 étamines transformées en autant de pétales.

Fr. Rosier pimprenelle à fleurs Ulanches demidoubles. - r. cultivé. 


\section{ROSA ARVENSIS MULTIELORA.}

(Ser. Ros, dess. Déc. 5. n.0 45.)

Obs. 1. Simple variété multiflore de la $R$. ar. vensis $\mathrm{L}$. (Ser. Ros. dess. n. ${ }^{\circ}$ 1.) Souvent on trouve sur le mème individu des rameaux 1, 2, 3, 6-flores.

Obs. 2. Dans les jets très-vigoureux de ce Rosier, qui, sur-tout lorsqu'il a été fortement taillé au printemps fleurit en automne, on trouve plusieurs bractées à la base d'un ou deux des pédoncules centraux, ce qui avec le grand développement du rameau lui donne un aspect étrange. C'est cet état que Mr. DeCandolle a nommé R. bibracteata Fl. fi. 5. p. 537. J'ai vu (Herb. DeCand) les exemplaires donnés par Mr. Bastard, Mr. Chaillet m’en a envoyé sous le même nom, j'en ai trouvé moi-même, et malgré des fleurs en corymbes, de doubles bractées, qui ne se rencontrent que dans les rameaux centraux, et qui sont dues à la vigueui du jet, de grandes fleurs (dues encore à la vigueur du jet), et rosées, des pédoncules garnis de poils glanduleux très-courts, (caractère de la $R$. repens Willd.) je suis convaincu que ce n'est qu'une variété de la $R$. arvensis L. R. arvensis bracteosa Ser.

Fr. Rosier des champs multifore. - Lo. Haies des chemins. 
46. ROSA SEMPERVIRENS SCANDENS.

(Ser. Fos. dess. Déc. 5. n.0 46:)

R. Sempervirens Lin. 1.p.704.n. ${ }^{0} 9$. - Willd. Spec. 2. 2. p. 1072. no 1S. - DeC. Cat. Hort. Monsp. p. 138. n.0 2. Fl. fr. 5. n.0 3696. a. p. 533. (var. SCANDENS.)

Obs. Malgré que cette espèce me semble trèsbien caractérisée par ses folioles fermes, épaisses, persistantes, par des styles réunis en colonne velue, par les glandes pédicellées de ses pédoncules (caractère que je n'ai jamais rencontré daus aucune des variétés de la $R$. arvensis); j’ai encore de très-légers doutes, (voyez Mus. helv. Red. Ros. 11. ${ }^{0} 6$ et 32.) Je désirerais en avoir en autornne des individus vivants, que je voudrais cultiver en Suisse dans un terrain humide et ombragé.

Fr. Rosier toujours vert. - All. Immergrüne Rose. - L. Avignon.

47. ROSA BREVISTYLA GLANDULOSA

(Ser. Ros. dess. Déc. 5. n . $^{0}$ T.)

Obs. 1. La réunion des styles en une colonne glabre et courte, caractère que je n'ai encore remarqué dans aucun rosier suisse, mais que je crois essentiel, m'engage à rapporter ma plante d'Aar- 
berg à la $R$. brevistyla DeC. Fl. fi. 5, 1n. 03714. b.; mais je suis fort peu sûr de ce rapprochement. Lorsque j'cus lc bonheur de passer quelques jours, l'année dernière auprès de Mr. DeCandolle, je n'avais pas autant travaillé les Roses, et je ne pris pas sur la $R$. brevistyla, dont je ne possède pas d'exemplaire, les notes nécessaires.

Obs. 2. Ma plante ressemblerait beaucoup à la 2. sepinm des auteur's si elle n'avait pas les siyles réunis en colonne courte. Fille a les folioles obovélancéolées, glanduleuses en dessous et odorantes comme la $R$. rubiginosa et sepium des auteurs, les pétioles couverts de poils lymphatiques ct glanduleux, les stipules plutôt étroites que larges, acérées et glanduleuses coinme les folioles, les pétioles courts, lisses, ainsi que le tube du calice, qui est ovoïde, les fleurs rosées, les aiguillons à base large et recourbés vers le sommet.

Obs. 3. C'est encore une des espèces sur lesquelles jai le pius grand besoin de l'aicie de mes collaborateurs, et je saisis cette occasion de les prier instamment de m'envoyer leurs remarques sur les Roses et sur ma Critique des Roses desséchées, ainsi que des exemplaires des espèces rares ou communeses, spontanées ou cultivées.

Fr. Rosier à styles réunis en colonne courte. - L. Aarberg. 
43. ROSA VILLOSA.

(Ser. Ros. dess. Déc. 5. n.0 48.)

Fruit jeune, provenant d'un individu cultivé, et -qui, dans l'état de maturité, acquert le volume d'un œuf de pigeon. Il est semblable à la figure qu'a donnée Mr. Redoute (Ros. Livr.4. p. 67.), mais il est plus violâtre qu'elle ne le réprésente.

\section{ROSA ALPINA LAEIIS.}

(Ser. Ros. dess. Déc. 5. n.0 49.)

R. alpina pedunculo glabro DeC. Fl. fr. 5. p. 536. n. 0 1712: $a$.

Obs. Simple variété a péduncules et calices entièrement lisses, tandis que les pétioles, la nervure centrale en sont garnis, (comme dans presque toutes les variétés de la $R$. alpina.), ainsi que les folioles et les stipules, qui en sont bordées.

Fr. Rosier des Alpes à péduncules et calices lisses. - L. Schwarzwasser.

50. ROSA ALPINA vULGARIS VARIEGata.

Obs. Variation accidentelle de la $R$. alpina vulgaris (Ser. Ros. dess. n. ${ }^{0} 6$.) - Cette bigarrure des feuilles ne se manifeste qu'à l'effloraison; auparavant 
les folioles sont d'un verd égal, et c'est à l'approche de l'effeuillaison qu'elle se manifeste d'une manière très-tranchée.

Fr. Rosier des Alpes à fezilies panuchées, L. Cultivé.

J'espère pouvoir par la suite offrir quelque chose de plus satisfaisant et de méthodique sur les Roses? mais j'ai pensé qu'il était nécessaire auparavant de príparer les changemens indispensables dans ce genre par quelques idées que j'envoye en éclaireurs. - Si je n'avais pas fait ce premier travail, je n'aurais pu avancer mes études ultérieurs pour la monographie des Roses, que je prépare.

La Monographie des CÉRÉALES DE IA SuIsse cupera le $n^{\circ} 2$ de ces Mélanges. 


\section{A T A L O G UE}

des espéces renfermées dans les cinq premières décades de

R OSES DESSÉ CHÉES.

\section{D É C A D E I.}

1. Rosa arvensis uniflora glabra (étiquetée R. procumbens.)

2. cinnamomea $\mathrm{L}$.

3. tomentosa Smith.

4. moschata semiplena (étiq. R. moschata.)

5. indica semperflorens semi-plena (étiq. $R$. semperflorens Willd.)

6. alpina vulgaris (étiq. R. alpina.)

7. rubiginosa vulgaris (étiq. $\mathrm{R}$. rubiginosa.)

8. rubrifolia laevis (étiq. R. rubrifolia.)

9. canina L.

10. rubiginosa sepium (étiq. R. sepium.)

\section{D É C A D E II.}

11 Rosa eglanteria lutea (ćtiq. R. eglanteria.)

12. eglanteria bicolor.

13. sulphurea multiplex (étiq. R. sulphurca fl. pleno.)

14. farolina hispida (étiq. $R$. carolina.) 
15. Rosa centifolia muscosa multiplex (étiq. $\mathrm{R}$. muscosa fl. pleno.)

16. centifclia multiplex rubra (étiq. $R$. centifolia flore pleno.)

17. culendarum multiplex alba (étiq. R. calend. A. pleno.)

18. calendarum multiplex alba (étiq. R. calend f. pl. albo,)

19. centifolia pomponia major. (étiq. R. pomponia maj. fl. pleno.)

20. centifolia pomponia minor. (étiq. R. pomponia minor $\mathrm{fl}$. pleno.)

\section{D É C A E III.}

21 Rosa gallica remensis violacea, (étiq R. remensis.) 22. gallica multiplex (étiq. R. gallica fl. pleno.) 23. gallica versicolor (étiq. R. gallica variegata f. pleno.)

24. gallice holosericea (étiq. R. holosericea.)

25. turbinata multiplex (étiq. R. turbinata fl. pleno.)

26. alpina latifolia (étiq. R. pendulina.)

27. indica chinensis scmiplena (étiqg. R. chinensis 1. pleno.)

23. fraxinifolia Gmel.

29. cinnamomea multiplex (étiq. R. cinnamomea fl pleno.)

30. a!ba multiplex (étiç. R. alba fl. pleno.) 


\section{D É C A D E IV.}

31 Rosa alba semi-plena.

32. gallica inaperta.

33. gallica pumila.

34. gallica hybrida.

35. canina collina.

36. canina nitens.

37. tomentosa glabriuscula.

38. tomentosa lanceolata.

39. indica humilis.

40. rubiginosa major.

\section{D É C A D E V.}

41. Rosa lucida Ehrh.

42. pimpinellifolia macrophylla.

43. pimpinellifolia myriacantha.

44. pimpinellifolia semi-plena alba,

45. arvensis multiflora.

46. sempervirens scandens.

47. brevistyla glandulosa.

48. villosa (fruct. jun.)

49. alpina laevis.

50. alpina vulgaris variegata, 


\section{TABLEAU ALPHABÉTIQUE}

des especes de Roses, variétés ou synonymes de cette CRITIQUE.

Nota. Les noms imprimés en caractères italiques sont ceux des espèces ou variétés des décades, ceux en caractères ordinaires sont les synonymes, et enfin les noms qui, après le $\mathbf{N} .^{\circ}$, ont le mot $O b s_{\text {, }}$, ne sont que les espèces ou variétés citées en observation. - Les chiffres renvoyent au N. ${ }^{0}$ des Roses de cette Critique et des Roses desséchées et non aux pages.

Agrestis Savi n. 7 . obs. 2.

Schleich. 10. obs. 2 .

Alba multiplex. 30.

semi-plena. 31 .

Alpina L. 6. - 26. obs. - 28. obs. 1.

laevis Red. 28.

laevis Ser. 49.

latifolia 25.

multiplex 6. obs. 2.

pedunculo glabro. DeC. 49

pendulina 6 . obs. $2 .-26$.

vulgaris 6 .

vulgaris variegata 50. 
Altaica Willd. 43. obs. 4.

Arvensis L. 1. - 46. obs.

bracteosa Ser. 45. obs. 2.

multiflora 45.

uniflora glabra 1.

Austriaca Crantz 22.

Belgica carnea rubro striata. Rœss. 23.

Bicolor Jacq. 11.

Blanda Ait. 28.

Borkhausii Gaud. 41.

Brevistyla DeC. 47. obs. 1.

glandulosa 47.

Burgundica Gmel. 21. - Pers. 20.

Calendarum flor, alb. pleno 18.

fl. pleno roseo 17.

Gmel. 17. - 20. obs. 1. - 32. obs. 2.

multiplex alba. 18.

multiplex rosea 17 .

Campanulata Ehrh. 25.

Canina L. 9.

collina 9. obs. 2. -35 .

dumetorum Desv. 35.

glabra Desv. 9.

nitens 9: obs. 2. -36.

pilosiuscula Desv. 37

sepium DeC. 10.

tomentosa 2. obs. 1.

Carolina L. 14.

corvmbosa Red. 14.

hispida 14.

laevis 14. obs.

Centifolia 2. obs. 1. $-16 .-20 . ; 6^{\circ}$. 
Centifolia carnea 32. obs. 1.

Kingston-Portugal 20. 3.

miniata 20. $40^{\circ}$

multiplex Red. 16. $-20.60^{\circ}-61$

multiplex rubra 16.

muscosa multiplex 15.

pomponia major 19. - 20. obs. 3,4.$20.5 .^{\circ}$

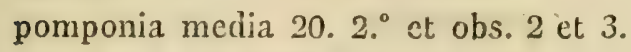

pomponia minor $20.10^{\circ}$

Cerea Rocss. 11.

unguiculata 23. obs. 2.

Chamaerhodon Vill:? 33.

Chinensis Willd. 27. - 39. obs. 2. - 27.

Chlorophylla unicolor Ehrh. 11.

Cinnamomea L. 2. - 14. obs. - 25. obs. 1. majalis Red. 29.

multiplex 28.

Collina Jacq. 35.

Collincola Ehrh. 2.

Corymbifera Borkh. 35. - Ehrh. 14.

Damascena subalba 17, obs. -

Dubia Wibel. 3. obs. 3.

Dumetorum Thuill. 35.

Eglanteria L. 7. - 11 .

bicolor 12.

lutea 11.

punicea 12.

Fotida 11.

Flava Lobel 7 .

Francofurtensis Desv. 25.

Fraxinifolia Gmel. 28. 
Gallica a. $\operatorname{Lin} 22 .-20$ obs. 1. - 21. obs. 1. 24. obs.

agatha 32 . obs. 2.

flore pleno 22.

holosericea 24.

hybrida 33.

inaperta 32.

multiplex 22.

pumila 33.

remensis violacea 21.

variegata 23. obs. 1.

versicolor 23.

versicolor marmorea 23 . obs. 2.

Geminata Rau. 34.

Glaucophylla Ehrh. 13.

Herporhodon Ehrh. 1.

Holosericea Lob. 23. obs. 1. - 24.

Hybrida Schl. 34.

Hall. Enum. p. 348. n. $0^{2}$. $-6 .=348 . n \cdot 0^{0} 3 .-$

33. $=349$. n. ${ }^{0} 6 .-1 .=350$. n. $7 .-7$.

Hall. Hist. n. 1101. a. $-9 .=1102 .-1 .=1103$.

$-7 .-11 .=1104 .-33 .=1105 .-3$.

$=1107 .-6$.

Indica acuminata 39. obs. 2.

chinensis semi-plena. $2 \%$.

humilis 39.

semperflorens semi-plena 5.

vulgaris Red. 27.

Inermis Mill. 6. - Delaun. 6. obs. 2.

Lagenaria 6. obs. 1.

Lucida Ehr. 41.

Lutea Ait. 11. 
Lutea multiplex Bauh. 13.

Miniata Hort. Bern. 20. 4. ${ }^{0}$

Mollissima Gmel. 3. obs. 3.

Moschata semiplena 4.

Mundi Andr. 23. obs. 1.

Muscosa Willd. 15. - 20.6.0

flore pleno 15.

multiplex Red. 15.

Myriacantha DeC. 43.

Myrtifolia Hall. fil. 10.

Nitens Hall. fil. 36 .

Omnium calendarum Rœss. 17.

Opsostemma plenum Ehrh. 4.

Parvifolia Ehrh. 21.

Pendula Lin. 26.

Pendulina Ait. 6. obs. 2. -26.

Pimpinellifolia macrophylla. 42.

myriacantha 43.

semi-plena alba 44.

Pomponia DeC. Fl. 20. - DeC. Herb. 19. - Red. 20. $20^{\circ}$ - obs. 3 et $30^{\circ}-$ Ser. $20.10^{\circ} 20^{\circ}$

kingston-portugal 20 obs. 3 et $3 .^{\circ}$

major fl. pleno. 19.

Prænestina plena Mill. 23. obs. 1.

Prostrata DeC. 1.

Provincialis a Willd. 19. - B. 20.

Puberula Desv. 34.

Pumila Clus et Jacq. 33. -24 obs.

pannonica f. rubello. J. Bauh. 33.

Punicea Roessig 12.

Pyrenaica 6. obs. 1.

Remensis DęC. 21. 
Repens Willd. 1. - 45. Obs. 2.

Rubra flore semi-pleno Bauh. 22.

Rubiginosa Lin. 7. - 12. obs. - 10. obs. - 4 T.

obs. 2. -

glabra Rau 10.

major 40.

resinosa Schl. $\tau$. obs. 1.

sepium 10.

tenuiglandủlosa 7 . obs. 2.

triflora 7 . obs. 2 et 3 .

vulgaris Willd. $\uparrow$.

Rubrifolia Vill. 8 .

laevis $\mathrm{S}$.

Rupestris Crantz. 6.

Sanguisorbac majorisfolio, finciu longo, longo pendula Dill. 26.

Scotica Desf. 43. obs. 4.

Semperflorens Desf. et DeC. it. - Willd. 5. -

39. obs. 2. -

Sempervirens Lin. 46.

postrata- Desv. 1.

scandens 46.

Sepium Thuill. 10. - 47. obs. 2.

ambigua ? Desv. 10. abs. 2.

Serpens Ehrh. 1.

Sinensis praecoxinermis 6 . obs. 2 .

Spinosissima 42. obs. - 13. obs. 2.

nana flore albo mutipl. And. 4 is.

Stylosa Desv. 1.

Sulphurea Ait. 13.

flore pleno 13.

multiplex 13. 
Sylvestris rubella parvofrutice J. Bauh. 33 .

Temuiglandulosa Mérat 7. obs. 3.

Tomentosa Smith 3.

glabriuscula 37.

lanceolata. 3. obs. 3. -38 .

lurbinata flore pleno 25.

$$
\text { multiplex } 25 .
$$

Umbellata Leers 7 . obs. 3.

Versifolia Gaud. 2. note.

Villosa Lin. 48.

mollissima Willd. 3. obs. 3.

Vilmorina 32. obs. 1.

Virginiana Duroi 14. 


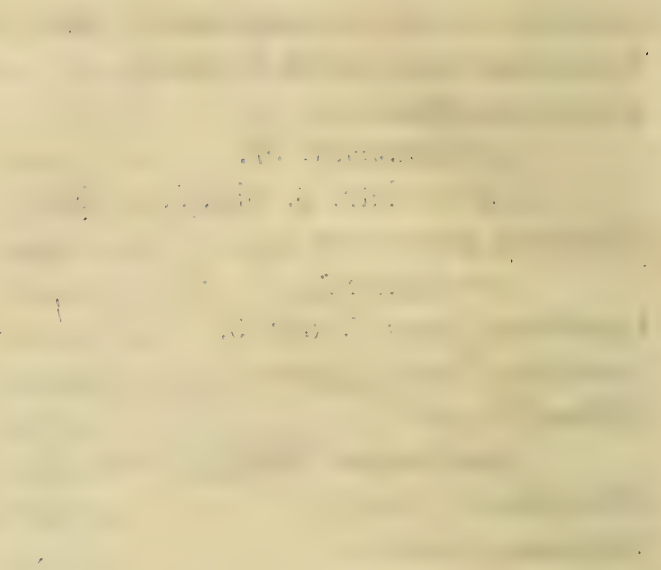




\title{
MÉLANGES BOTANIQUES
}

ou Recueil d'observations, mémoires et

\author{
notices sur la botanique, \\ par N. C. Sertange.
}

N. 2.

Septembre 1818.

MONOGRAPH I E

DES

CÉRÉALES DE LA SUISSE.

La culture de la plupart des Céréales $\left({ }^{*}\right)$, qui appartiennent à la farnille des Graminées, et à la Triandrie Digynie de Limné, est tellement répandue, qu'on ignore actuellement quelie fut leur patrie. Elles font la vraie richesse d'un pays, et ont de tout temps occupé un grand nombre d'hommes. Le sol, le climat, les engrais les ont tellement changées, qu'il est assez difficile actuellement d'en rap-

(*) Blés, seigle, orges, avoines, etc. etc. 
porter les différentes variétés aux vraies espèces. Les pays de plaines, plus faciles à être travaillés, et où elles murissent plus facilement, ne sont cependant pas les seuls où elles soient cultivées; les gras et riches pâturages de quelques-unes des verdoyantes vallées alpines sont souvent interrompus par des champs de blé, de seigle, d'avoine et d'orge. Là, où la proximité des hautes Alpes amène de continuelles vicissitudes, où la saison favorable à leur végétation est de courte durée, il a fallu se borner à semer quelques espèces, qui murissent plus vite; et ce sont en général les blés à graines enveloppées (section des épeautres) qui semblent s'accommoder le mieux aux climats froids. Aussine rencontre-ton guère en Suisse, surtout dans le voisinage des Alpes, que le Triticum spelta, amyleum et monococcum, joints au seigle, à l'avoine et à quelques orges. Les blés à graines nues ou les froments ne sont généralement cultivés que dans le canton d'Aargovie, et dans la partie française de la Suisse. $\left(^{*}\right)$

(*) Dans quelqques vallées alpines, où la neige reste longtemps, on pourrait peut.être employer le procédé sui. vant, noté par Mr. DeCandolle : (Rapport sur un voyage botanique et agronomique, 1810 p.99.) „Pour accélérer le moment où il est possible de cultiver et d'ensemencer le terrain, certains habitans, et notam. ment ceux de la vallée de Chamouny, ont imaginé un procédé industrieux, destiné à hâter la fonte de la neige. Au commencement du printemps ils répandent sur la neige, qui couvre leurs champs, de la poussière de schistes noirâtres, préparée à cet effet dès l'été précédent; cette poussière échauffée par le soleil, fond la neige autour d'elle, hâte ainsi de quinze jours le moment 
Les Céréales servent non seulement à la nourriture de l'homme, mais encore à celle de presque tous les animaux domestiques. La paille courre le toit du pauvre, sert de litière aux bestiaux, puis d'engrais; elle est employée à la fabrication des chapeaux, des nattes. Les Céréales sont done un objet d'utilité première, et dont il nous serait absolument impossible de nous passer. Llles sont quelque fois aussi cultivées pour fourage, et servent en vert à la nourriture des bestiaux.

La difficulté de parfaitement rendre par le dessin et la gravure, la forme des épis des Céréales m'a fait naitre l'idée de faire sous le nom d'Herbier Céréal, une collection d'épis murs, non comprimés et de Céréales comprimées, séchées arant leur maturité; ce moyen facilitera j'espère leur étude.

\section{DISTRIBUTION DU MÉMOIRE.}

\section{$10^{\text {re }}$ PARTIE.}

Introduction à l'étude des Céróales.

La terminologie botanique étant peu connue des agriculteurs, auxquels ce travail est en partie destiné, j'ai joint à la deffinition des organes des Céréales différentes remarques qui leur sont relatives.

où la surface du sol est accessible, et en se déposant même sur le terrain, à moitié décomposée, et chargée de petites immondices, qui s'y joignent, elle contribue l'engraisscr." 


\section{PARTIE.}

Description des genres, espèces et variétés des Céréales.

Cette portion du mémoire est presqu'entièrement du rapport de la botanique. J'y ai donné les caractères des genres, des espèces, des variétés, en y rapportant les synonymes avec le plus de soin qu'il m'a été possible. J'y ai joint les noms vulgaires français, allemands etc., et, sous le titre d'Observations, diverses notes critiques.

\section{Partie.}

\section{Maladies des Céréales.}

Je n'ai fait dans cette troisième partie que présenter le résultat très-succint de ce que l'on sait sur les maladies des blés; l'on trouve dans l'Herbier Céréal des exemplaires de toutes ces maladies.

\section{Partie.}

\section{Usages économiques des Céréales.}

J'ai tâché dans cette portion du mémoire, de donner une idée des préparations, que l'on fait subir aux Céréales pour les approprier à nos besoins, et dans le but de mieux faire connaître les farines, grueaux, préparation des pailles etc., j'ai pris auprès des meuniers, des fabricans de chapeaux de paille etc., ce qui me semblait pouvoir intéresser la partie économique. 


\section{INTRODUCTION À L'ÉTUDE DES CÉREAALES.}

\section{1. " PARTIE.}

Les principales difficultés, qui se sont toujours offertes à l'étude des Céréales, viennent de ce qu'on a souvent regardé comme caractère spécifique:

1. La présence ou l'absence des arêtes;

2. la villosité ou glabréité des glumes ou des glumelles;

3. la couleur de ces mêmes glumes et glumelles;

4. l'apparence bisannuelle de quelques variétés.

En un mot, qu'on ne s'est attaché le plus sourvent qu’à des caractères de simples variétés.

Je crois qu'avant d'indiquer les différentes espèces et-leurś caractères, il convient de noter les parties les plus essentielles de ces végétaux, afin de tâcher de mieux me faire comprendre, surtout des personnes qui ne connaissent pas la botanique. En voici le tableau:

$$
\begin{gathered}
\text { Organes } \\
\text { de la } \\
\text { végétation. }
\end{gathered}\left\{\begin{array}{l}
\text { A. Racine, (Radix, Wurzel) } \\
\text { B. Chaume, (Culmus, Halm.) } \\
\text { C. Feuille, (Folium, Blatt.) } \\
\text { D. Epi, (Spica, Aehre.) } \\
\text { E. Epillet, (Spicula, Locusta, Aehr- } \\
\text { F. Panicule, (Panicula, Rispe.) } \\
\text { G. Rachis, Rafle, (Rachis, Spindel.) }
\end{array}\right.
$$




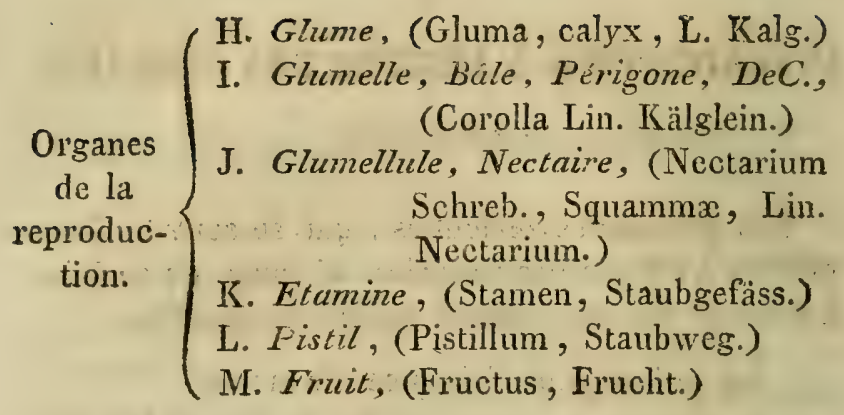

Organes de la végétation.

A. Racine, (Radix, et dans les composés grecs Rhizos, partie de la plante, située à son extrémité inférieure, ordinairement cachée sous la terre, qui tend toujour's à descendre ver's le centre du globe, ne se colore (presque jamais) en vert, par l'action de la lumière, sert à fixer la plante au sol et à pomper sa nourriture. DeC. Théor. p. 325.)

Les racines des Céréales sont ordinairement fibreuses. Les grains des blés, orges et avoines, d'après Mr. DeCandolle, naissent avec trois radicules, tandis que toutes les autres plantes connues n'en ont qu'une.

Toutes les Céréales sont annuelles; ce n'est que pour hàter leur maturation, et être plus sûr des récoltes que nous les semons en automne, et que nous les transformons en apparence en plantes bisannuelles; mais cultivées sous un climat convenable, on trouvera qu'elles sont toutes annuelles. Dans les années favorables à la végétation même en Suisse, 
par exemple en 1818, je suis sûr que semées au printemps, elles auraient toutes réussi, mais ce serait trop s'exposer; c'est par cette raison qu'on séme la majorité des Céréales en automne. A cette époque les labours se font mieux, la graine germe, la plante s'enracine, talle, développe une partie de ses feuilles avant l'hiver, et par la faculté qu'elle a de résister à des froids d'une certaine durée, reste sans croître pendant les rigueurs de l'hiver; puis an printemps pousse avec d'autant plus de force, que les racines sont bien établies. Par cette mème raison la fleuraison a lieu plutot, et les Céréales traitées de cette manière, peuvent mieux supporter les vicissitudes de l'air, qui retardent souvent leur maturité.

Au printemps au contraire la terre très-humide se travaille souvent moins bien, souvent elle est moins bien ameublie, les racines s'y établissent moins facilement, la plante est moins vigoureuse; et si, à ces désavantages, se joignent un été défavorable, une fleuraison difficile, par les froids, les pluies, la récolte est médiocre, mauvaise, ou plus rarement nulle.

Je suis donc persuadé, qu'un observateur sans préjugés, ou qui cherchera la vérité, trouvera que toutes les Céréales que nous cultivons, sont réellement annuelles. Le Trit. durum B., l'un des blés qui a le plus besoin d'une terre bien fumée, et de chaleur, a été semé cette année dans le Simmenthal, au printemps, et il $y$ a parfaitemcut réussi ; 
la graine avait été enroyée par le gorvemement; elle venait d'Égypte; on l'a scmée par hasard au printemps, et comme cette année a été très - favorable, et que la plante a réussi, on ne manquera pas de dire dans le Simmenthal, si quelques bonnes années se succèdent, que c'est une espèoe de printemps. Ma variété du Triticum vulgare que les Vaudois appélent blé motu, est regardée comme une plante de printemps; je l'ai semée en autoinne, elle a parfaitement réussi, a muri de tres-bonne heure, et si, inconnue d'abord dans une contrée, on la semait en automne, elle serait réputée comme Céréale d'automne. Je le répète, toutes nos Céréales sont annuelles, nos préjugés seuls en rendent quelques-unes bisannuelles: toutes cultivées avec des circonstances favorables, murissent très-bien, semées au printems, mais il vaut mieux faire nos semis dans les deux saisons, afin d'être moins exposés à une foule d'accidents, qui pourraient nous priver de notre première nourriture. La culture des blés en Égypte vient encore à l'appui de ce que j'ai avancé. Plusieurs cultivateur's en Suisse sèment l'épeautre au printemps, resèment en automne la même graine, qu'ils ont obtenue en été, et en font une Céréale d'hiver.

B Chaume (culmus), Tige cylindrique, munie d'espace en espace de nouds compacts, desquels les feuilles prennent naissance, et propre à la famille des graminées.

Le chaume est fistuleux ou plein, terne ou lustré, paille, roux ou rarement violâtre ou rougeâtre. 
J'ai dans ma collection des épis, dont ce chaume est violet d'un côté et paille de l'autre, ou paille dans la partie enveloppée par la gaîne, et violette au-dessus; dans d'autres le chaume est violet et l'épi couleur paille. J'ai quelque idée qu'il est plus constamment fistuleux ou creux; il faudrait voir s'il ne pourrait pas concourir avec d'autres caractères à fixer des espèces. On range surement sous le nom de Triticum vulgare des plantes qui ne lui appartiennent pas.

C. Feville (Folinu). Expansion ordinairement plane, verte, horizontale, qui nait sur la tige des plantes, sert à l'évaporation et à l'imbibition des vapeurs et des gaz nutritifs, et est formée de l'épanouissement d'une ou de plusieurs fibres. DeC. Théor.p. 332.

Toutes les feuilles des Céréales ont des nervures longitudinales, au lieu de pétiole une gaîne (vagina) fendue, qui part du noud (nodus) placé audessous d'elle, et entre la gaine et la partie laminée une ligule ou languette (ligula), qui n'est qu'un appendice membraneux, qui couronne cette gaîne.

Toutes les parties des froments et épeautres, en contact avec la lumière, sont couvertes particulièrement à l'époque de la fleuraison, et même quelque temps après, d'unc légère efflorescence glaucescente, qui disparait avant la maturation.

D. ÉpI (Spica). Assemblage de fleurs sessiles le long d'un axe central persistant, et à peu près vertical: Dẹ. Théor.p. 358. 
La grande majorité des Céréales a des fleurs en épi, cet épi est quadrilatère, distique ou hexastique, glabre ou velu, barbu ou mutique, couleur paille, roux ou noiràtre, penché ou droit. La forme et la direction de l'épi offrent des caractères constants dans chaque espèce; mais sa couleur, son velu, son aristation ou sa muticité ne sont que des caractères de variétés.

E. Epillet (Spicula, Locusta). Petit épi, dont les fleurs sont solitaires ou clisposées sur deux rangs, et renfermées originairement dans une glume. C'est le nom qu'on applique á chacun des petits épis, dont se compose l'épi général, ou la panicule des graminées. DeC. Théor. p. 359.

Ces épillets sont quadri, bi ou uniflores, tous ou en partie fertiles.

F. Pantcure (Panicula), se dit des fleurs, qui étant en grappe, à pédicelles rameıx, ont les pedicules inféricurs allongés, écartés ou très - rameux. DeC. Théor. p. 359.

Les espèces des genres Avena et Milium offrent cette espèce d'inflorescence. Cette panicule est étalée, pyramidale, penchée, unilatérale etc.

G. RAchrs, Rafle (Rachis). Pédoncule centrat ou axe d'un épi ou d'une grappe; la fin de la tige dans le genre Triticum par exemple où sont implantés les épillets.

Ce Rachis est tenace dans les froments (Frumenta) 
et fragile dans la section des épeautres(Speltæ); dans toutes les especces des genres Triticum, Secale, Hordeum, il est formé de petites articulations au sommet desquelles sont implantés alternativement les épillets; souvent ces articulations, qui communiquent des uns aux autres par des espèces d'arètes vives, sont glabres ou garnies de poils.

\section{Organes de la reproduction.}

C'est particulièrement dans les organes de la reproduction des graminées en général, que les dénominations abondent, ce qui a en partie augmenté la difficulté, qu'offre l'étude de la famille des Graminées, par l'embarras de pouvoir entendre les auteurs. C'est dans ces organes que se trouvent les meilleurs caractères d'espèces, mais encore faut-il ne se servir que de ceux qui sont vraiment constants.

H. Glume (Gluma* ${ }^{*}$ ). Calix L. - Glume calicinale, Glume extérieure. - Bâle (Tegmen) de Beauv, - Lépicène (Lepicena) Rich. Espèce d'involucre, situé à la base de l'épillet, renfermant zune ou plusieurs fleurs, composé ordinairement de

(*) J'ai pensé qu'en adoptant les mots très-simples de Glume (Gluma), Glumelle (Glumella, bàle), Glu. mellule (Glumellula, nectaire); ce serait simplifier la nomenclature, et que cette conformité des syllabes radicales rappélerait en même temps l'analogie, qui existe entre toutes ces parties. 
deux pièces inégales, situées de manière que l'une est toujaur's insérée un peu au-dessus de l'autre. DeC. Théor. p. 367.

Cette Glume dans les froments est tantot glabre, tantot velue (elle est presque constamment glabre dans la section des épeautres); elle est souvent aussi teinte dans la même espèce de diverses couleurs; conséquemment cette villosité, cette glabréité, cette couleur n'offrent que des caractères de variétés; mais sa carène, le mucrone qui n'en est que le prolongement (lequel est très-rarement nul dans le genre Triticum, où il ne se transfọrme presque jamais en longue arête), offrent de bons caractères d'espèces.

I. Glumelle (Glumella) Desu., corolla Lin. Bâle de beaucoup d'autres. - Glume intérieure, glume corolline. - Périgone DeC. - Stragule, (Stragula) de Beauv. -

Espéce de périgone de nature et de structure analogue à la glume, mais propre à chaque fleur, et situé autour des organes génitaux. DeC. Théor. p. 368 .

Cette Glumelle offre de bons caractères de groupes et d'espèces : dans le genre Triticum, par excmple, groupes des froments des Français, la graine tombe nue sous le fléau, en laissant la Glume et la Glumelle adhérentes aurachis, (Semen maturum valvutis denudatum; rachis fexilis. Rom. et Schult. Syst. 2. p. 761.); et dans la section des épeautres 
(froment des Suisses), la Glumelle s'applique sur la graine à la maturité, et tombe avec elle, le rachis étant fragile (Semen maturum valvulis calycinis corticatum; rachis fragilis. Rom. et Schult. Syst. 2: p. 766.)

Les épillets du genre Triticum, sont quadriflores dans les froments aussi bien que dans les épeautres; mais souvent une ou deux des fleurs du centre dans l'épillet avortent, et alors les Glumelles en restent plus petites et plus minces: Dans l'état parfait d'un épi du genre Triticum, la Glumelle externe de chaque fleur est constamment terminée dans son état parfait par une longue arête hérissée de poils ascendants, de la même nature que la Glumelle; (la valve interne toujours plus mince, moins dure, plus plane, en est privée, sa face interne répond au sillon de la graine); mais cette valve externe perd souvent son arète (arista) par avortement partiel ou total, soit dans les fleurs centrales d'un épillet, soit même dans les latérales; et un épi plus ou moins pourvu de toutes ses arêtes, ou qui en est entièrement privé, doit naturellement offrir un aspect bien différent. Ces arêtes commencent à avorter, surtout dans le genre Triticum, du centre à la circonférence de l'épillet, puis de la base au sommet de l'épi; à mesure que ces arêtes diminuent de longueur, le mucrone de la valve externe de la Glume diminue aussi. La perte ou avortement des arêtes, en partie ou en totalité, tient à des causes que je crois encore inexpliquables; mais sur lesquelles des expériences réitérées pourront peut-être un jour donner 
quelque lumière, si l'on se réprésente $10^{*}$ un épi, dont la valve externe des Glumelles est pourvue d'arète; $2 .^{\circ}$ un autre, dans lequel les deux arêtes des deux fleurs latérales existent seules; $3 .{ }^{0}$ celui dont une partie de ces arêtes des fieurs latérales est avortée ; $4 .{ }^{0}$ celui dans lequel la base en est totalement dépourvue, les Glumelles du son،met seules, en conservant quelques-unes, toujours très-courtes aloi's; $5 .{ }^{0}$ celui qui est entièrement mutique, et que l'on joigne à tout cela la couleur, la villosité, la glabréité, non moins variables, des Glumes et Glumelles surtout dans les froments, on conviendra facilement que l'on a cherché à établir des espèces sur des caractères de simples variétés. Je suis d'ailleurs très-persuadé, que lorsqu'on étudiera bien soigneusement les blés, les orges, les avoines, le seigle même, on en trouvera tôt ou tard des variétés entièrement mutiques.

J. Glumellule, Glumellula Desv. Corolle. Micheli. - Ecaille Lin. - Nectaire, Schreb. - Giumelle. Rich. - Lodicules, (lodicula) de Beauv.

Espèce de Nectaire situé autour du pistil, manquant dans plusieurs graminées et composé de trèspetites écailles glumacées souvent ciliées.

Jusqu'à préscnt j’ai cherché à me fraycr une marche dans l'étude des Céréales; mais il m'a échappé beaucoup d'objets, dans lesquels on pourra peut-être trouver des caractères importants. Je n'ai encore rencontré que dans les orges à graines nues ces Glumellules; elles y sont très-visibles sur le 
sec, mais je me propose à l'avenir de donner plus de soin à l'étude des étamines, des pistils et des feuilles.

K. Etanine (Stamen.). Organe mâle des plantes, ordinairement situe autour du pistil et composé de l'Anthère (Anthera) et du filet (filamentum.)

Dans toutes les Céréales les étamines sont hypogynes (naissant de dessous l'ovaire) et sont ordinairement au nombre de trois, conséquemment de la Triandrie de Linné; (le Maïs est monoïque.) Les Anthères sont oblongues, fourchues aux deux extrémités, et souvent pendantes par l'arcuation de leur débile filet.

La fleuraison des blés est d'une très-grande importance pour obtenir des récoltes abondantes; des froids, des pluies continuelles à cette époque rendent la plante faible; le pollen est lavé, la fécondation se fait mal ou très-imparfaitement, conséquemment la récolte est mauvaise. On dit alors que les blés ont coulé, ce qui signifie que la fécondation s'est mal opérée.

On a généralement blâmé la culturc des Céréales mêlées, que les agriculteur's appélent méteil, mais je crois que dans des pays où les récoltes sont souvent incertaines, on s'expose moins à des récoltes décidément mauvaises, si l'on cultive le méteil. Deux espèces de Céréales flcurissent rarement ensemble, si l'une coule, l'autre réussira peut-ĉtre, fleurissant dans un moment plus favorable. On pourrait semer un blé à graines nues avec un au- 
tre à graines enveloppées, on les séparerait facilement ensuite par le crible; et si l'une murit un peu avant l'autre, on pourrait avancer de quelques jours la moisson, pour l'espèce qui est la plus avancée, sans nuire cependant à l'autre. C'est en effet ce que pratiquent quelques agrieulteurs dans plusieurs parties élevées de la Suisse.

L. Pistil (Pistillum). Organe femelle, situé au centre de la fleur, et formé $1 .{ }^{0}$ inférieurement de l'ovaire (ovarium, germen), qui renferme les rudimens des jeunes graines, lesquelles avant leur fécondation portent le nom d'ovules (ovula, ova); $2 .{ }^{0} \mathrm{du}$ style (stylus), prolongement de l'ovaire qui supporte le stygmate (stigma), qui est la troisième partie du pistil, ordinairement situé au sommet et où la matière fécondante de l'organe mâle vient se déposer. DeC. Théor. p.369,

Peut-être trouverait-on aussi dans l'une des parties du pistil quelques bons caractères; le stigmate dans la plupart des Céréales est fendu en deux parties et est plumeux.

M. Fruit (Fructus, et dans les composés grecs Carpon.)

Dans l'usage vulgaire ce mot désigne ordinairement les fruits charnus et mangeables; mais dans le sens exact du mot, il signifie tout ovaire fécondé etc. DeC. Théor. p. 376.

Ce fruit est un cariopse nu ou recouvert par la Glumelle (bâle), l'embrion est petit, attaché à la base 
base d'un périsperme farineux, plus gros que lui. Entre ces deux organes se trouve une plaque charmue, regardée par Gartner comme un vitellus, et par Jussieu comme un cotyledon, qui ne se développe point à l'époque de la germination. DeC. Fl. fr. vol. 3. p. 2 .

Le Cariopse (Cariopsis Rich.) est un fruit sec, monosperme, et dont le péricarpe est tellement adhérent, qu'il se confond avec le périsperme, ou enveloppe propre de la graine.

Ces deux enveloppes de la graine des Cérćales, nommées péricarpe (la plus exlerne), périsperme (située au-dessous), produisent, lorsqu'on écrase ces graines, le péricarpe, le gros son; le périsperme, le petit son. Au-dessous se trouve le périsperme farineux, qui fournit à l'homme la plupart des farines qui font la base de sa subsistance.

Quand on aura bien étudié les graines des Céréales l'on y trouvera je crois dans le rapport de leur longueur à celui de leur largeur, dans l'angle que forme le sillon longitudinal qui les traverse, leur plus ou moins grande convexité, l'obtus, l'aigu ou le tronqué de leur extrémité, des caractères d'espèces. 
DES C R I P T I O N

DES GENRES, ESPÈCES ET VARIËTÉ

DES CÉRÉALS.

$20^{\circ}$ PARTIE.

\section{T R I T I C U M.}

T. Floribus spicatis; rachi flexuosa, compressa, articulata; spiculis alternis in summis articulis sessilibus; gluma bivalvi, cymbiformi, mucronata, quadriflora; glumella bivalvi; seminibus ovoïdeis, ellipsoïdeis vel triquetris, sulcatis.

\section{B L É.}

Fleurs en épi; rachis flexueux, comprimé, articulé; épillets alternes, naissants du sommet de chaque articulation; glume bivalve, naviculaire, mucronée, quadriflore; glumelle bivalve; graines ovoïdes, ellipsoïdes ou triangulaires, sillonnées. 
Le genre Blé, Triticum, offre deux sections bien naturelles; dans la première, sous le nom de Froments, Frunienta, se trouvent les espèces, dont les graines tombent nues sous le fleau (dépourvues des valves de la Glunelle, qui restent adhérentes au rachis non fragile). Cette section est d'autant meilleure, qu'elle est appuyée sur un autre caractère bien tranché, c'est d'avoir les graines ovoïdes ou ellipsoïdes, et le sillon qui les traverse profond. La section des Epeautres, Speltce, dont les graines tombent enveloppées par les deux valves de la Glumelle, qui les tiennent étroitement embrassées, (le rachis se rompant à chacune de ses articulations), est appuyée sur des graines triangulaires, dont une des faces est traversée, par le sillon peu profond qui se remarque toujours sur le grain. 


\section{TA B L EA U}

DES ESPECES ET VARIÉTÉS DES FROMENTS.

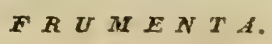

Semibus maturis denudatis; rachi flexili; gluma qua. đrifora; floribus tribus fertilibus; seminibus ovoïdeis vel ellipsoïdeis.

\section{Triticum vulgare.}

Spica tetragona, adscendente ; spiculis abbreviatis ; gluma wentricosa, ad apicem compressa; seminibus ovoïdeis, obtusis, farinosis, opacis.

A. Spica laxa, aristata, alba, glabra.

B. Spica laxa, aristata, alba, velutina.

C. Spica laxa, aristata, rufa, glabra.

D. Spica laxa, aristata, rufa, velutina.

E. Spica laxa, mutica, alba, glabra.

F. Spica laxa, mutica, alba, velutina.

G. Spica laxa, mutica, rufa, glabra.

H. Spica laxa, mutica, rufa, velutina.

I. Spica compacta, aristata, alba, glabra.

J. Spica compacta, mutica, rufa, glabra. 


\section{Triticum turgidum.}

Spica tetragona, inclinata; spiculis abbreviatis; gluma ventricosa, abbreviata, late mucronata, carina compressa; glumella inflata; seminibus ovoïdeis, gibbis, farinosis, opacis.
A. Spica aristata, alba, velutina,
B. Spica aristata, rufa, velutina.
C. Spica aristata, rufa, glabra.
J. Spica aristata, nigra, velutina, densa.
E. Spica aristata, nigra, velutina, laxa.
F. Spica mutica, velutina.
G. Spica ramosa, velutina.

\section{Triticum durum.}

Spica tetragona, inclinata; gluma elongata, late mucronata; carina compressa, lateribus planis; glumella concaviuscula, longe aristata; seminibus ellipsoldeis gib. bis, corneis, semipellucidis.
A. Spica aristata, velutina.
B. Spica aristata, rufa, glabra.
C. Spica aristata, glabra, versicolori.

\section{Triticum polonicum.}

Spica tetragona, compressa vel laxa; gluma longis. sima, bidentata; valvula externa florum lateralium lon. giore; seminibus longe ellipsoïdejs, subtriquetris.

A. Spica compressa, aristata, villosa.

B. Spica laxa, divaricata, aristata, glabriuscula. 
SECTION PREMIÈRE.

Frumenta

Semibus maturis denuda= tis; rachi flexili; gltuna quadriflora, floribus tribus vulgo fertilibus; seminibus ovoüdeis vel ellipsoideis.
SECTIO PRIMA.

Froments.

Graines tombant nues à leur maturité, la glumelle restant fixée au rachis nonfragile; glume quadrittore, dont trois sont ordinairement fertiles; graines ovoïdes ou ellipsoïdes.

\section{TRITICUM VULGARE. (*)}

Spica quadrangulari, adscendente; spiculis abbreviatis; gluma ventricosa, ad apicem compressa; seminibus ovoïdeis, obtusis, farinosis, opacis.

Epi quadrilatère, ascendent; épillets courts; glume ventrue $\left({ }^{* *}\right)$, carène comprimée au sommet, s'éva-

(*) Cette espèce et ses nombreuses variétés, si cultivées en France et dans d'autres pays, ne le sont guère en Suisse que dans les cantons de Vaud, Neuchâtel, Bâle et Aargovie. Les meuniers à Berne trouvent que le Trit. vulgare rend plus de farine que le Trit. Spelta, mais les boulangers préfèrent ce dernier, la farine exigeant moins de travail pour sa panification. D'ailleurs le froment ordinaire, dont les graines sont nues, se conserve moins facilement que l'épeautrc.

(**) Terminée (dans cette espèce) par un long mucrone acéré, lorsque la valve externe de la Glumelle est aristée; obtuse au contraire lorsque cette valve externe de la Glumelle est mutique. 
nouissant vers la base; graines ovoïdes, obtuses, farineuses, opaques. $\left(^{*}\right)$

1. Trit. vulgare. ( $T$. aeslivum $L$.)

A. Spica laxa, aristata, alba, glabra.

A. Epi lâche, barbu, blanc et glabre.

Triticum aristis longtoribus, spica alba. C. Bauh. Pin. - Vaill. Bot.p. 196. n.0 4. et Rai Hist. p. 1239. (**)

Triтicum. Tournef. Instit. 3. tab. 293.

Tritrcum aestrvum Lin. Hort. ups. 21. - Lin. Spec. 1. p. 126. - Gouan Fl. monsp. 128. n. 1. Kerner œhonom. Pflanz. tab. 292. (fig. méd.) Lagasca, Gen. et Spec. n. ${ }^{0}$ 87. (d'après Rom. et Schult. Syst. 2. p. 761.) - Loisel. Fl. gall. 1.p. 70. n.01. - Roem. et Schult.Syst. 2.p. 761. - Schweigger et Kcerte. Fl. Erlang. p. 31.n.0 1. - Ser. Herb. rur. Déc. 21. n. ${ }^{0}$ 6. - Sut. Fl. helv. 1.p. 75. n. ${ }^{0} 2$.

- Willd. Spec. 1.p. $476 .-$

(*) Aucune des variétés du Trit. vulgare n'a, en Suisse, de chaumes pleins au sommet, tandis que les quatre Touzelles, (toutes glabres, deux aristées, blanche et rousse) deux mutiques, (blanche et rousse) ont une tendance à avoir plus ou moins des chaumes pleins. Les Touzelles de Mr. Dunal appartiennent bien certainement au Trit, vulgare, mais je ne sais si le climat pourrait produire cette différence dans le chaume.

(**) A l'exemple de Mr. DeCandolle, j’ai cité les synonymes par ordre de date; mais quand un auteur n'a fait qu'adopter la dénomination donnée par un autre, je l'ai cité par ordre alphabétique pour faciliter les recherches. 
TRITICUM LOCUSTIS IMBRICATIS, QUADRIFLORIS , ARISTATIS Hall. Hist. $n \cdot 0^{0}$ 1422. $B$.

Triticum Sativum Lam. Dict. 2.p. 554. $=$ DeC. Fl. fr. 3.p. S0. n. ${ }^{0} 1656$. Synops. Fl. gall. $n .{ }^{0} 1656$. - Dum. de Cours. Bot. cult. éd. 2. Tom. 2. p. 109. - Gaud. Agrost. 1.p. 337.var. a. - Pers. Syn.1. p. 109, n.0 1., a.

Triticum vulgare Vill. Fist. 2. p. 153. $z .=$ Host. Gram. 3. T. 26. (var. aristée, médiocre). Willd. Enum. 1. 133. var. $a$.

Touzelle blanche, barbue Dum.ined. (d'aprés les exempl. qu'il m'a envoyé en 1817.)

FrG. Tournef Inst. 3. Tab. 293.

Kerner okonom. Pflanz. Tab. 292.

Host. Gram. 3: Tab. 26.

Ser. Herb. cer. n. ${ }^{0}$ 1. A. (maturum.)

Cette variété ne se trouve que rarement en Suisse, et presque toujours mêlée avec d'autres.

Franc. Froment commun, barbu, blanc et glabre. - Blé grison. Hall. Hist. et Nov. Comm. Froment d'automne à épi blanc. DeC. Fl. fr. 3. n. 0 1656. p. 80. var. a: - Blé trémois $\left(^{*}\right)$ Touzelle blanche, barbue, Saisette. (Dunal. Montpell:)

(*) Ce mot vient probablement de la corruption des mots trois mois, à causc du temps de sa durée. 
Allem. Sommer-Wcizen. Willd. Spec.

Gemeiner Weizen mit grünen Kernen.

Angl. Wheat oder Weet-Spring wheat. -

Sommer-wheat.

1. TRIt. vUlgare (T. astivum var, $L_{*}^{*}$ )

B. Spica laxa, aristata, alba, velutina.

B. Epi làche, barbu, blanc et vclouté.

TRITICUM Y.OCUSTIS QUADRIFLORIS, BASI SUEHIRSUTIS; GLUMIS EXTERIORIBUS FLORALIBUS ARISTATIS. Irall. Nov. comment. 5. p. 5. n. ${ }^{0}$ 6. Tab. 1. fig. 1. (un seul épillet est figuré.)

FIg. Hall. Nov. Comment. 5. T. 1. f. 1. (épillet.) Ser. Herb. cer. n. $^{\circ}$ 1. B. (matur.)

Cette variété se trouve aussi rarement que la précédente dans les champs de la Suisse. (*)

Franc. Froment commun, barbu, blanc et velouté.

1. Trit. vulg. (T. astiviem var. $L$.)

C. Spica laxa, aristata, rufa, glabra.

C. Epi lâche, barbu, roux et glabre.

(*) Il serait ridicule de chercher à désigner plus minutieusement, que je le fais, les diverses variétés. Le velouté, qui se remarque plus ou moins fortement sur les Glumes et les Glumelles, varie beaucoup; elles en sont plus ou moins couvertes, et la partie convexe est 'célle qui en' est le plutôt privée. 


\section{0}

ToUzelle rovge barbue, SaISETte de tarascon. (Dunal. exempl. murs.)

Fig. Ser. Herb. cer. n. ${ }^{0}$ 1. C. (matur.)

Ça et là dans les champs, mais rarement en Suisse.

Franc. Froment commun, barbu, roux et glabre, Touzelle rouge, barbue, et Saisette de Tarascon, (d'après Mr. Dunal. exempl. murs.)

1. Trit. vulgare (T. ocstivum. var. L.)

D. Spica laxa, aristata, rufa, velutina.

D. Epi lâche, barbu, roux et velouté.

FIG. Ser. Herb. cer. n. ${ }^{0}$ 1. D. (matur.)

Se rencontre quelquefois dans les moissons de la Suisse.

Franc. Froment commun, barbu, roux et velouté.

1. Trit, vulgare (T. hybern, $\left.L_{0}\right)$

E. Spica laxa, mutica, alba, glabra.

E. Epi lâche, sans barbes, blanc et glabre.

Tritrcum Tournef. Inst. 3. T. 292.

Trit. hybernum. L. Hors. ups. 21. Spec. 1. p. 126. n.0 2. - Gessn. Tab. plyttogr. T. 7. fig. 94. n. ${ }^{0}$ 2. - Gouan. Fl. Monsp. 128. - Kern. ockon. Pflanz. T. 293. - Lagasca Gen. et spec. n.0 86. 
d'après et avec Roem. et Schult. Syst. 2.p.'763. $n^{0}{ }^{0}$. - Loisel Fl. gall. 1. p. 70. n. ${ }^{0}$ 1. - Sut. Fl. helv. 1. p. 74. n.0 1. - Willd. Spec. 1. p. 477. n.0 2. -

Triticum vulgare d. Vill. Hist. 2. p. 153.

Triticum tovzelle Vill. Hist. 2. p. 154. n. ${ }^{0} 2$.

Triticum vulgare hybernum Host. Gram. 3. Tab. 26. (mutica, médiocre.) - Willd. Enum. 1. p. 133.

Tritrcum satrvum B. spiculis loevilus, plerumque muticis. Gaud. Agros. 1. p. 337. -

Triticum vUlgare aUtumale Spica alba seminibus aureis Tess. $n .{ }^{0}$ 1. (d'aprè's et avec Rom. et Schult. Syst. 2. p. 762. var. a. (*)

T'ouzelle blanche sans barbe (Dun. exempl. mur.) $\left({ }^{* *}\right)$

Fig. Gessn. Tab. phytogr. T. 7. f. 94. n: 2

Kern. œkon. Pflanz. Tab. 293.

Host. Gram. 3. Tab. 26. (mutica.)

Ser. Herb. cer. n. ${ }^{0}$ 1. E. (matur.)

(*) MM. Rcem. et Schult., qui ont regardé le $T$. asti= vusn et hybernum comme deux espèces, et qui en ont fait consister les caractères en la présence ou l'absence des arêtes, auraient aussi dû répartir les variétés aristées dans leur Trit. æstivum, et celles qui étaient sans barbes dans le Trit. hybernum.

(**) Très-cultivée à Montpellier, mêlée avec la Touzeile blanche, barbue; semée en automne. (Mr.. Dunal.) 
Assez fréquemment cultivé dans les cantons fiontières de la France.

Franc. Froment commun sans barbes, blanc et glabre. - Froment sans barbes, à épi blanc et à graines jaunes, Dum. de Cours. Bot. cult. édit. 2. vol. 2. p. 109. (sans descript.) Froment d'automne à épi blanc Tess. DeC. Fl. fi. 3. p. So. 11.0 1656. var. a. Touzelle blanche sans barbe.

Allem. Winter-Weizen. Willd. Spec.

1. TRIT. Vulgare (T. hybern. var. L.)

F. Spica laxa, mutica, alba, velutina.

F. Epi lâche, sans barbe, blanc et velouté.

TrtTCUM aristis carens, gLUmis pubescentrbus Vaill. Bot. p. 196. $n .{ }^{0} 2$.

Triticum hybernum molee Fell. Inst. d'agr.

Triticum vUj,gare aUtuminale Spica mutica, glumis villosis cinereis Tess. $n_{0}{ }^{0}$ \%. (d'après Ræm. et Schult. 'Syst. 2. p. 762. m.

Fig. Ser. Herb. cer. n. ${ }^{0}$ 1. F. (matur.)

Variété assez commune dans la partie française de la Suisse.

Franç. Froment commun sans barbes, velouté, blanc. - Blé de Bohême. - Froment grisâtre, épi velouté, graines dorées etc. DcC. Fl. fi. 3. p. 81. var. du n'0 1656 . $=$ 
Allem. Böhmischer Sammtweizen. Fell. Inst. d'agr.

1. Trit. vulgare $(T$. hybern. L.)

G. Spica laxa, mutica, rufa. glabra.

G. Epi lâche, sans barbes, roux et glabre.

Triticum spica et granis RUbentibus Vaill. Bot. p. 196. n.0 3.

TRITICUM LOCUSTIS IMBRICATIS QUADRIFLORIS GLABRIS, MUTICIS Hall. Hist. n. ${ }^{0}$ 1422. a.

Trimicum sativum DeC. Fl. fr. 3.p. $80 . n .01656$. var. $B$.

Triticum vulgare autumnale Spica rubra, seminibus aureis Tess. $n .{ }^{0}$ 22. (d'apre's Rom. et Schult. Syst. 2. p. 762. var. $\ell$.

Blé tammas Lamour: Rapp. p. 4.

Touzelle pouge sans barbes Dun. (ex.mur.)

Fig. Ser. Herb. cer. n. $0^{\circ}$. G.

Cultivée assez fiéquemment dans la partie française de la Suisse.

Franc. Froment commun sans barbes, glabre et roux. - Froment d'aulomne à épi doré. DeC. Fl. fi. 3. p. 80. n. ${ }^{\circ}$ 1656. var. B. Froment sans barbes à épi doré et à graines jaunes. Dum. de Cours. Bot. cult. éd. 2. vol. 2. p. 109. (sans descript.) - Touzelle rouge sans barbes. Dunal. - Blé Lammas.

Allem. Winterweizen. 
1. Trit. vulgare ( $T$. hybern. var, $L$, )

H. Spica laxa, mutica, rufa, velutina.

H. Epi lâche, sans barbes, roux et velouté.

Triticum locustis gUadrifloris, GLABRIS, Bast subhirsutis, glumis sub muticis. Hall. Nov Com. 5. p. 7. n. 7. T. 1. f. 2. (mêlé avec mon n. ${ }^{0}$ 1. f.)

Frg. Ser. Herb. cer. n. ${ }^{\circ}$ 1. H. (matur.)

Mêlée dans les champs de la Suisse fiançaise avec les autres variétés du froment cominun; semée en automne.

Franc. Froment commun sans barbes, velouté et roux.

1. Trit. vulgare (T. compactum Host.)

I. Spica compacta, aristata, alba, glabra.

I. Epi compact, barbu, blanc et glabre.

Trit. compactum Host. Gram Aust. 4. T. 7. (var. aristata.)

Fig. Host. Gram. Aust. 4. T. 7. (var. Arist.)

Obs. 1. Je viens de recevoir de Mr. de Haller fils, des épis murs avec l'étiquette de Trit. sardinicum Host. qui se rapportent parfaitement à cette variété $I$, et qui ressemblent entièrement à cette variété barbue du Trit. compactum.

Obs. 2. Voyez pour la synonymie et les observations, la variété suivante (J.)

Franc. Blé ordinaire varićté à épi compact et barbu. Allem. Binkel Weizen. Host. 1. c. 


\section{5}

1. Trit. vulgare (T. compactum Host.)

J. Spica compacta, mutica, rufa, glabra.

J. Epi compact, sans barbes, roux et glabre.

Tritrcum compactum (var, mutica.) T. spica compacta, spiculis aristatis, internodiis racheos brevissimis, foliis vaginisque inferioribus scaberrimis. Host. Gram. 4. T. 7. var. mutica. - Ræm. et Schult. Syst. 2. p. 765. n. ${ }^{0}$ 14. (En excluant le $T$. sativum pyramidale. Descript. de l'Egypte T.14.)

Triticum creticum Schweik. (Morell. coll. de Céréales.)

Triticum sativum DeC. Fl. fr. 3.p. $80 . n \cdot{ }^{0} 1656$. var. froment d'Alsace.

Frg. Host. Gram. Aust. 4. T. 7. (var. mutica.)

Obs. 1. La table 7. vol. 4. des Graminées d'Autriche de Mr. Host, réprésente trois figures; deux ont les épis aristés, et répondent à la variété précédente, la troisième ressemble parfaitement à mon Trit. muticum compactum n. ${ }^{\circ}$ 1. I. ; l'épi parait tronqué au sommet, où il conserve le plus souvent quelques arêtes courtes.

Obs. 2. Cette variété, semée au printemps, cultivée à Hofwyl, et dans plusieurs parties du canton de Berne, a été envoyée à feu Mr. Morell, il y a environ douze ans, par Mr. Schweikert de Carlsruh. Ses épis, quoique courts, rendent à peu près autant de grains que ceux de l'autre race: l'épi ne parait si court qu'à cause de la briéveté des 
articulations du rachis. D'ailleurs les proportions de toutes les parties des épillets sont parfaitement les mêmes, que dans les variétés précédentes.

Obs. 3. Je n'ai encore vu que deux variétés de cette race à épi compact, l'une barbue, blanche et glabre, qui est rare, et l'autre mutique, rousse et glabre, qui est cultivée en grand dans les cantons de Fribourg et Vaud, sous le nom de Blé-mottu; mais en l'observant soigneusement on trouvera surement toutes les variétés de la race à épis alongés.

Obs. 4. Je possède des exempl. précieux, qui oflient le passage d'une race à l'autre. La base de l'épi est làche, et les épillets de la partie supérieure entassés. Quant à moi je n'ai pas le moindre doute sur l'identité des deux races. Le Lolium perenne offre un même exemple d'entassement des épillets.

Obs. 5. Il est bien certain que le Trit. sativum pyramidale Delile Fl. xgypt. n. ${ }^{0} 155$. , et Descript. de l'Egypte, 2. Livr. partie de l'hist. natur. T. 14. f. 3. ne peut être rapporté ni à l'une ni à l'autre des races de mon 'Trit. vulgare, comme l'ont fait (avec doute, il est vrai) MM. Rœm. et Schult, Syst. 2. p. $765 . n^{0}{ }^{14}$., car l'épi du T. sativurn pyramidale est figuré au moins une fois plus large, se termine insensiblement en pyramide, et n'est pas tronqué au sommet; les Glumes et Glumelles sont trìsgrosses, le grain est aussi figuré plus gros que cclui du T. Lurgidum $\mathrm{L}_{\text {. }}$, presque rond et velu. I)'aillcurs les noeuds du chaume sont renflés, le bas de ce chaume 
chaume est tuberculeux; les feuilles à peu près de la largeur de celles du $T r$. turgidum, mais manifestement canaliculées à la manière de celles des jacinthes; il est probable que ce sera au T. turgidum quil sera rapporté un jour arec certitude.

Franc. Froment commun barbu à épi compact. Froment d'Alsace. DeC. Fl. fr. 3. p. 80. Froment sans barbes, d'Alsace, à épi court. Dum. de Cours. Bot. cult. éd. 2. vol.2. p. 109. (sans descript.) - Blé de Crète. - Blé mottu. -

Allem. Cretischer Weizen. - Sommer-Weizen aus Esula. Morell (Collect. de Céréales du Musée d'hist. natur. de Berne.) - Binkel Weizen. Host. Gram.

\section{TRITICLM TURGIDUM.}

Spica tetragona, inclinata; spiculis abbreviatis; (") gluma ventricosa, abbrevi.ta, late mucronata; carina compressa; glumella inflata; seminibus ovoideis, gibbis, farinosis, opacis.

Epi tétragone, penché; épillets courts; $\left({ }^{*}\right)$ glume ventrue, courte, terminée par un large mu-

$\left(^{*}\right)$ Des quatre fleurs de chaque épillet, trois sont ordinairement fertiles, rarement toutes les quatre; mais lorsque deux gıaines avortent l'épi est moins régulièrcment tétragone, mais toutes les parties d'ailleurs n'en ont pas moins la mềme forme. 
crone; carène comprimée dans toute sa longueur; glumelle renflée; graines ovoïdes, bossues, farineuses, opaques.

2. Trit. turgidum. (Pétanielle blanche.)

A. Spica aristata, albà, velutina.

A. Epi barbu, blanc et velouté.

Trit. turgidur Vill. Hist. 2. p. 155. n. 03 .

Trit. sativum var. Pétanielle blane. DeC. Fl. fr. 3. n. ${ }^{0} 1656 . p$. 81 .

Trit. aestivun o. b. spica aristisque albicantibus. Rom. et Schult. Syst. 2.p. 762.

Petantelle blanche et froment blanc de Montpellier. Dunal inéd. (Epi mur.)

Obs. Cette fort belle variété à épi blanc se trouve très-rarement mêlée avec les autres dans le canton de Vaud, où l'espèce est fréquemment cultivée, elle se sème aussi en automne.

Franc. Pétanielle blanche, froment blanc de Montpellier, moutin blanc, blé d'abondance.

2. Trit, turgidum (Pétanielle rousse.)

B. Spica aristata, rufa, velutina.

B. Epi barbu, roux et velouté.

Triticum aristatum, spica maxima cinericea, glums Hnsutis Rai Hist, p. 1238. 
TRITicum SPICA villosa gUadrata breviore et tURGIDIORE Moris. Hist 3 p. 17G. sect. 8. T. 1. fig: 14 .

Trit. turgidum Lin. Hort. ups. 21. Spec. 1. p. 126. - Gaud. Agrost. 1.p. 338. n.0 2. - Host. Gram. 3. Tab. 28. - Loisel. Fl. Gall. 1. p. 70. n.0 4. - Rocm. et Schult. Syst. 2. p. 763. n.0 5. (*) Sut. Fl. helv. 1. p. 75. n.0 4. - Willd. Spec. 1. p. 478. n. 4. Enum. 1. p. 133. n. ${ }^{0} 3$.

TRIT. LOCUSTIS QUIDRIFLORIS HIRSUTIS, BAST PILosis Hall. Nov. Com. 5. p. 12. n. ${ }^{0}$ 9. (var. 2. glumis floralibus aristatis.)

Trit. sativem DeC. Fl. fr. 3. p. 81. n.0 1656. var. Pétanielle roux.

Trit. polonic uni (non L.) $\gamma$. turgidum. Pers. Syn. 1. p. 109. n. $0^{0} 3$.

Trit. aestivus o. a. Trit. vulgare autumnale, spica brevi, crassa, turgida, aristata, rufescente; culmo farcto Rom. et Schult. Syst. 2. p.762.

Petanielte rousse, froment roux de Montpellier. Dunal inéd. (Epis murs.)

Fig. Moris. Hist. 3. sect. 8. T. 1. fig. 14. - Host. Gram 3. T. 25. - Ser. Herb. cer. 2. B.

Obs. 1. Cette variété réussit très-bien dans les

${ }^{*}$ ) En excluant la Synonymie de la Descript, de l'Egypte , Tab. 14. 
terres fortes et très-fumées. Elle produit de trèsgros grains et en grande quantité. Elle préfère une exposition un peu chaude; c'est pour cette raison qu'elle est beaucoup plus cultivée dans le canton de Vaud et en Italie, qu'à Berne, où des essais faits dans des années défavorables, et l'apauvrissement du terrain l'ont fait abandomer.

Obs. 2. La figure citée de Host. Gram. 3. T. 28. ne domne pas une idée exacte du volume de l'épi, car il a été figuré à l'époque de la fleuraison, et il grossit beaucoup pendant la maturation.

Obs. 3. Je ne conçois pas trop l'idée de $\mathrm{Mr}$. Persoon, qui a regardé le Trit. turgidum L. pour une variété du Trit. polonicum; cette dernière espèce est une des plus tranchée du genre, et je n'ai jamais rien vu qui lui ressemblat.

Obs. 4. Je ne puis croire, avec MM. Rom. et Schult. Systema 2. p. 763. n. ${ }^{\circ}$ 5., que la Table 14. fig. 2. du magnifique ouvrage intitulé : Description de l'Egypte, (que possède la Bibliothèque de Berne) réprésente le Trit. turgidum L., à moins que le dessinateur n'ait figuré l'épi beaucoup plus gros et plus court qu'il ne l'est ordinairement. Outre cela dans cette gravure l'épi est très - droit et nullement penché, comme dans notre T. turgidum; les feuilles quoique planes sont aussi plus larges que dans notre espèce; le chaume est plein, (ce qui est commun aux deux plantes); les glumes et les valves de la glumelle, (quoique plus volumineuses et réprésentant assez bicu le $T$. turgidum L.) sont aussi velucs. 
Voici la remarque que fait Mr. Delile Hist. des plantes cultivées en Egypte, 1. ${ }^{\text {er }}$ Mémoire, Céréales, p. 13. "Il n'y a en Egypte que du blé barbu. Son " chaume s'élève un peu moins que celui du même " blé (T. turgidum), cultivé en France. Les variétés " nommées Qamh meghayz, qamh naygeh ( $T$. sati"vum pyramidale, Tab. 14. fig. 3.), et qamh a'raby "( $T$. sativum turgidum, Tab. 14. fig. 2.), sont "inconnues en France." Aux mots $T$. sativum turgidum se trouve la synonymie de $T$. durum Desfont. qui, si elle est juste, me porterait encore plus à croire, que ces deux plantes d'Egypte sont différentes de notre Trit.turgidum, que Mr. Desfontaines connait très-bien, et qui est certainement distinct de son Trit. durum. (*)

Franc. Gros blé, blé de Sicile.. Pétanielle rousse. Froment renflé. F. roux de Montp. Blé angl.

Allem. Englischer Weizen.

Espag. Redondillo. - Arab. Qamh a'raby.

2. Trit. turgidum (Pétanielle rouge.)

C. Spica aristata, rufa, glabra.

C. Epi barbu, roux et glabre.

Petanielle rouge, froment rouge de montpellier Dunal inéd. (épi mur.)

Trit. linnaeanum? Lagasca, (d'aprés Rom. et Schult. Syst. 2. p. 763. n.0 4.)

(*) M. Desfont. m'a envoyé parmi d'autres exempl. précieux, un blé rapporté de Gizé par Mr. Delile, avec le nom Arabe de Qamh mraéz, que je ne puis séparer de cette variété. 
Obs. Cette variété se trouve mêlée avec les autres Pétanielle, dans les moissons des environs de Montpellier. Je l'ai aussi trouvée dans celles du canton de Vaud, mais un peu plus rarement que la plupart des autres variétés de cette mème espèce. La longueur de l'épi ne varie pas moins (de 6-50 graines) que dans toutes les espèces et variétés de blés, selon qu'on les cultive dans des terrains très-fumés, bien travaillés, ou bien qu'on les sème dans des ter- , res maigres et mal labourées etc. etc.

Franc. Gros blé à épi roux et glabre. Pétanielle rouge. Froment rouge de Montpellier.

\section{Trit. turgidum.}

D. Spica aristata, nigra, velutina, densa.

D. Epi barbu, noir, velouté et serré.

FIG. Ser. Herb. cer. 2. D.

Obs. Même forme, même velouté que la var. 2. B. , mais l'épi est noir. Cette variété se trouve dans les moissons du canton de Vaud, mêlée avec les autres.

Franc. Gros blé à épi noir et compact.

2. Trit. turgidum (Pétanielle noire.)

E. Spica aristata, nigra, velutina, laxa.

E. Epi barbu, noir, velouté et lâche. 
TRIT. SATIVUM VAR. FROMENT GRIS-DE-SOURIS DeC. Fl. fr. 3. n. ${ }^{0}$ 1656.p. 81 .

Petanielle noire, froment noir de montpellier Dunal inéd. (Epis murs.)

Obs. 1. Cette bclle variété, quoique au premier aspect extrèmement différente des aulres par ses épillets un peu plus écartés, et sa couleur d'un gris noir très-prononcé, apparticnt certainement au Triticum turgidum. Ses graines ont absolument la même conformation, et acquèrent plus de volume (dans toutes les variétés de blés), ou les épillets sont plus lâches, par la facilité qu'elles ont alors de se développer.

Obs. 2. Cette varićté offre d'ailleur's une foule de nuances, depuis l'épi et les arêtes noires, jusqu'à l'épi d'ui gris pâle, avec les arêtes rousses; ct depuis l'épi renfermant de 5 graines jusqu'à 56 . On la trouve quelquefois aussi passant dans la variété 2 . B. et $D$., par ses arêtes noirâires et ses épis roux, presque :glabres.

Franc. Gros blé noir. Pétanielle noire. Froment noir de Montpellier. Froment gris-de-souris. Gros blé noir à épillets écartés.

2. Trit. TURGIDUm.

F. Spica: mutica, velutina.

F. Epi sans barbes et velouté. 


\section{4}

TRIT. LOCUSTIS QUADRIFLORIS, HIRSUTIS, BASI pilosis; glumis exterioribus muticis Hall. Nov. Comment. 5. p. 12. n. ${ }^{0}$ 9. var. 1.

Obs. Je n'ai jamais vu la variété mentionnée par le Grand-Haller, mais plus cette espèce sera cultivée et bien étudiée, plus elle offrira de variétés.

Franc. Gros blé mutique, (sans barbes, sans arêtes.)

2. Trit. turgidum ( $T$, compositum.)

G. Spica ramosa, velutina.

G. Epi rameux et velouté.

TRITICUM CUM MULTIPLICI SPICA J. Bauh. Hist. 2. p. 407. (bonne figure.)

Trit. SPICA baSI ramosa Hall. Hist. (entre le n. ${ }^{0}$ 1422. et 1423. que l'on trouve désigné dans quelques ouvrages par la lettre grecque $\gamma_{0}$ )

TRIt. compositum Lin. fil. suppl. 115. (d'aprés Ram. et Schult. Syst.) $=$ DeC. Fl, fr. 3. p. 82. n.0 1657. - Delile Fl. agypt. Descript. de l'Egypte n. ${ }^{0}$ 156. - Gaud. Agrost. 1. p. 339. - Host. Gram. 3. Tab. 2T. - Loisel. Fl. gall. 2. p. 70. n.0 3. Pers. Syn. p. 109. n. ${ }^{0}$ 2. - Rom. et Schult. Syst. 2. p.763. n. ${ }^{0}$ 3. - Sut. Fl. helv. 1. p. 75. n. ${ }^{0}$ 3. - Vill. Hist. 2. p. 157. n. ${ }^{0}$ 5. - Willd. Spec, 1, p. 47 . Enum. 1. p. 133. 
Fig. J. Bauh. Hist. 2. p. 407.

C. Bauh. Pin. 17 .

Moris. Hist. 3. p. 175. S. 8. T. 1. fig. 7.

Host. Gram. 3. Táb. 27.

Obs. 1. Je suis actuellement très-ccrtain, comme lavait déjà pensé $\mathrm{Mr}$. DeCandolle, que le $T$. compositum des auteurs n'est qu'une simple variété du T. turgidum, dont la base se ramifie plus ou moins. J'en ai trouvé des individus à peine ramcux à leur base, et dont les épillets de la partie supérieure étaient absolument conformés comme ceux du $T$. turgidum.

Obs. 2. Avant de devenir rameux, souvent les ćpillets du $T$. turgidum se dérangent, et l'épi a un aspect hérissé, qui n'est que le passage du $T$. turgidum $\mathrm{B}$. à cette variété $\mathrm{G}$.

Obs. 3. "La qualité de son grain (remarque Villars, Histoire des plantes du Dauphiné 2. p. 157.) est tendre, délicat et le rend propre à certaines préparations de patisserie, la pâte en étant plus blanche, plus fine et moins susceptible de fermenter. Un autre avantage, c'est que cette espèce produit beaucoup plus qu'aucune autre; mais ce dernier est balancé par sa difficulté de murir dans nos climats, par la quantité d'engrais et par le choix du sol gras qu'elle exige. L'Italie offre une partie de ces avantages, aussi la plante $y$ est-elle cultivée depuis longtemps." Les essais en petit réussissent rarcment; les Glumelles restent ordinairement bvides par l'avortement des grains. 
Obs. 4. On fail gruer dans le canton de Vaud la plupart des variétés de cette magnifique espèce, pour en faire des soupes très-nourrissantes, et de très-facile digestion.

Franc. Gros blé à épi rameux. (*) Blé de miracle. Blé d'abondance. Froment à épi rameux.

Allem. Vielkörniger Weizen.

\section{TRITICUM DURUM.}

Spica tetragona, inclinata ; gluma elongata. late mucronata; carina compressa, prominente; lateralibus planis; glumella concaviuscula, longe aristata; seminibus ellipsoïdeis, gibbis, corneis, semipellucidis.

Epi tétragone, incliné; glume oblongée, terminée par un large mucrone; carène très-saillante, còtés planes; glumelle peu voutée, terminée par une très-longue arête; graines ellipsoïdes, bossues, cornées et demi-transparentes.

Obs. Cette belle cspèce à chaume plein, à trèslongucs arêtes, à épi ressemblant beaucoup plus (quant à l'imbrication et au saillant de la carène) au Trit. comyleum qu'à toute autre espèce de blé, a été distinguée du Trit. turgidum par Mr. Desfon-

(*) Je prie les cultivateurs, où cctte variété murirait bien, de m'en envoyer deux ou trois cents épis murs, non comprimés et bicn conservés. 
taines, dans sa Flora atlantica, sous le nom de Trit. durum, et par Mr. Host dans ses Icones et descriptiones Graminum austriacorum 4. T. 5. (*) La carène comprimée et saillante, qui sépare les deux $f_{a}$ ces planes de la glume, jointe aux graines plus longues que dans le Trit. turgidum, bossues au milieu du dos, et à leur consistance cornée, ne me laissent aucun doute sur la solidité de cette espèce. J'ai cru devoir adopter de préférence la dénomination de Trit. durum Desf., puisqu'elle a la primordialité sur celle de Trit. hordeiforrne; le caractère corné de la graine se retrouve dans les trois seules variétés que je connaisse, et il est peut-ètre tout-àfait propre à cette espèce.

3. Trit. DURUm.

A. Spica longe ari tata, velutina.

A. Epi longuemest b.rbu et velouté.

Trrt. DuRum. Caule farcto, glumis pubescentibus, seminibus du is; spiculis quadrifloris. Desf. Fl. Atl.1. p. 114. $\left(^{*}\right)$ - Trit. spica pyramidatoteretuscula longissima pubescente, glumis utrinque compressis latecarinatis bidentatis, semine longissi-

(*) Du moins la variété glabre et rousse; voyez les différentes variétés de cette jolie espèce.

(**) D'après un exempl. que m'a envoyé Mr. Desfon* taines, avec cette note : „ Le chaume est plein, la "graine ue donne presque pas de farine, il se réduit "en gruau par la mouture." 
mo. Lagasca Gen.et Spec. Nov.diagn.n.095. D'apres et avec MM. Rom. et Schult. Syst. 2. p. 765. n.0 11.

Trit. villosum Host. Gram. Austr. Tom. 4. Tab. 6?

Trit. vulgare palmare Delile. Fl. ogypk $\pi^{\circ}$ 152. $\left({ }^{*}\right)$

Trit. Sativum duRum, Froment de Barbarie. DeC. Fl. fr. 3. $\pi_{0}{ }^{0} 1656 . p .81$. - Pers. Syn. 1. p. 109. n.0.1.

Obs. Cette belle varićté, cultivée cette amnée dans le Simmenthal, par Mr. le pasteur Steck, de graines d'Egypte, que le gouvernement de Berne avait fait venir dans l'hiver de 1817 , a été semée ce printemps, et a parfaitement muri.

FIG. Ser. Herb. cer. 3. A. (matur.)

Franc. Blé corné, variété à épis barbus et veloutés, nommée froment de Barbarie. Froment dur.

Espag. Trigo Moruno.

3. Trit. DURUM (T. hordeiforme.)

B. Spica aristata, rufescente, glabra.

B. Epi barbu, roussâtre, glabre.

( ${ }^{*}$ D'après un exempl., récolté dans les environs de Rosette (lequel avait probablement été donné par Mr. Delile, qui a remis de ses céréales d'Egypte à $\mathbf{M r}$. Desfontaines), et que je tiens de l'auteur de la Flore atlantica. 
TRIT. HORDEIFORME, spica disticha, spiculis aristatis, aristis spica triplo longioribus, foliis glabris levibus. Host. Gram. Austr. 4. Tab. 5. - Rom. et Schult. Syst. 2. p. T65. n. ${ }^{0}$ 13. (qui citent aussi Tratt. Tabular. Tab.367.)

Trit. typhinum Dodonei Hist. p. 25. (d'après MM. Host., Rom. et Schult. l. c.)

FIG. Ser. Herb. cer. 3. B.

Obs. Cette intéressante variété n'a je crois encore été cultivée que par $\mathrm{Mr}$. de Haller fils à Interlacken, où elle réussit fort bien depuis deux ans; mais comme elle produit, quoique le grain soit gros, une petite quantité de farine noire, on pourrait peutêtre utiliser cette espèce en la faisant gruer, ou de toute autre manière.

Franç. Blé corné, variété à épis barbus et glabres.

3. Trit, Durum (Tangarock.)

C. Spica aristata, glabra, versicolori.

C. Epi barbu, glabre, panaché.

TANGaRock Dunal inéd. (Epi mur.)

Obs. Cette singulière variété de printemps, rarement cultivée en Provence, (d'apres ce que me marque Mr. Dunal), et qui a absolument tous les caractères de l'espèce, n'a point encore été, à ce que je sache, trouvée en Suisse, mais il est probable qu'on la trouvera parmi les céréales, que l'on cultive de graines reçues de l'Egypte et d'ailleurs. 
Franc. Blé corné, variété à deux couleurs. Tangarock des Provençaux.

\section{TRITICUM POLONICUM.}

Spica letragona, compressa vel laxa; gluma longissima, bidenlata; valvula externa florum lateralium longiore; seminibus longe ellipticis, subtriquetris.

Epi tétragone, comprimé ou làche; glume trèslongue, bidentée; valve externe des fleurs latérales manifestement plus longue que l'interne; graines longuement ellipsoïdes, subtriquètres.

Obs. Cette belle espèce offre beaucoup de caractères tranchés, tandis qu'on a souvent de la peine à en trouver de vraiment distinctifs dans les autres blés. La plante entic̀re est bleuâtre jusqu'à la maturation; le chaume plein; l'épi grand, tétragone, comprimé, très-régulièremeut ordiné, quand la plante a acquis son parfait développement; trèslàche ct divariqué dans les excmpl. crûs en Suisse; les épillets sont quadriflores; la glume grande, presque membraneuse, carinée, fréquemment nervée et terminée par deux petites dents ascendentes ct ćgales; la valve externe de la glumelle dans les deux fleur's extérieures est longue ct aristéc, mais toujours l'une plus longue que l'autre, cn alternant à chaque ćpillet; la valve interne de ces fleurs latérales est toujours manilestement plus courte que l'ex- 
Eerne; les deux valves de la glumelle de la 3." Heur sont égales en longucur, et mutiques; la $40^{\circ}$ enfin est stérile.

4. Trit. poisonicum.

A. Spica compressa, villosa, aristata.

A. Epi comprimé, velu et barbu.

Trit. polonicum Lin. Spec. 1. p. 127. n.0 4. Dunal (exempl. mur.)

Obs. Je n'ai jamais vu dans aucun herbier d'épi aussi régulièrement disposé que dans celui de $\mathrm{Mr}$. Dunal, je crois que c'est là son état parfait.

Franc. Blé de Pologne, variété à épi serré. Blé d'Egypte. Blé de Surinam. Blé de Mogador.

Ailem. Polnischer Weizen. Wallachisches Korn.

Espag. Trigo de Polonia.

4. Trit. polonicum.

A. Spica laxa, glabrizscula, aristata.

A. Epi lâche, presque glabre et barbu.

Trit. Levissimum, GLaucum, locustis quadriPLORIS DUOBUS FLORIBUS ARISTATIS, DUOBUS MUcronatis Hall. Hist. $n_{0}{ }^{0} 1423$.

TrIT. LEVISSIMUM, LOCUSTIS TRIFLORIS, FLORIBUS CAI.YCEM EXCEDENTIBUS, COMPRESSIS, LONGISSIMTE aristatis Hall. Nov. Comm. 5. n.0 14. p. 17. Tab. 1. f. 16. (très-bonne.) 
Trit. polonicum Lin. Spec. 1. p. 127. n.0 4. Willd: Spec. 1.p. 478. n. ${ }^{0}$ 5. Enum. 1.p. 133. n. ${ }^{0} 4$. - Gaud. Agrost. 1. p. 341.n.0 6. - Host. Gram. Austr. 3. Tab. 31. - Pers. Synops. 1.p. 109. n. ${ }^{0} 3$. $a$. - Roemer et Schult. Syst. 2. p. 766. n.0 17.

Fig. Moris. Hist. 3. p. 175. S. 8. T. 1.f. 8. (*) Hall. Nov. Comm. T. 1.f. 16. Host. Gram. 3. Tab. 31.

Obs. 1. Cetté variété a été plusieurs fois cultivée en Suisse, même anciennement, mais il parait qu'elle n'a jamais bien réussi. La divergence des parties de ses épis et la courbure des arètes vers leur base lui donnent un aspect fort étrange, mais tous les caractères de l'espèce s'y retrouvent.

Obs. 2. Les graines provenant du même ćpi, semées en automne, ont produit des épis divariqués; ceux du printems de cette année s'approchaient plus de la forme dense de l'épi parfait.

Obs. 3. Je suis bien persuadé, que si l'on cultivait en grand cette espèce, on verrait paraître une foule de variétés.

Franc. Blé de Pologne, variété à épi divariqué.

(*) Je n'ai pu vérifier cette citation, conséquemment je ne sais si la fig. de Morison se rapporte a la var. $\mathbf{A}$ ou $\mathbf{B}$. 
Les blés de cette section (Frumenta) ont un désavantage sur ceux à graines enveloppées (Speltce), en ce qu'ils sont souvent ravagés par les moineaux, inconvénient auquel les épeautres ne sont pas exposés, par la dureté et l'étroite application des glumelles sur les graines. Les calculs que font Mrs. Rougier de la Bergerie et Bosc, sur la déprédation que causent ces animaux, sont vraiment effrayants. D'après Mr. de la Bergerie il y a aumoins dix millions de moineaux en France, consommant chacun vingt livres de grain par an, ou un boisseau, évalué à un franc; ce qui donne un résultat, qui prouve que ces oiseaux coutent dix millions de francs à l'agriculture française. Selon Mr. Bose, on doit doubler cette quantité. Plusieurs observations positives constatent que le jabot d'un de ces oiseaux contient aisément à la fois cent grains de blé; or, digérant très-promptement, il est des circonstances où il peut se remplir deux fois par jour. En se réduisant à cette quantité, cela fait environ deux boisseaux par an. On a senti dans plusieurs pays le dégat que produisent ces oiseaux, aussi leur a-t-on fait une guerre très-opiniâtre en Angleterre et dans quelques parties de l'Allemagne. Les pies et les corbeaux ont souvent été enveloppés dans la proscription; mais il parait que le grain qu'ils mangent, ne peut être comparé à l'utilité dont ils sont, en débarrassant la terre des vers et des larves des hannetons. (Thiébaut de Berneaud, Biblioth. physicoéconom. Sept. 1818. p. 153.) 


$$
\text { S P E E I T A A E. }
$$

Seminibus maturis, ralvatis; rachi fragili; gluma qua. driflora; floribus duobus vel unicis fertilibus; seminibus triquetris.

\section{Triticum Spelta.}

Spica subtetragona, inclinata; spiculis laxe imbricatis: gluma truncata; carina subcompressa, rectiuscula, lateribus planiusculis; seminibus triquetris, longis, acutis, opacis; culmo cavo.

A. Spica aristata, alba, glabra.

B. Spica aristata, alba, velutina.

C. Spica aristata, rufa; glabra.

D. Spica mutica, alba, glabra.

E. Spica mutica, rufa, glabra.

F. Spica mutica, rufa, velutiria.

G. Spica mutica, violacea, glabra.

H. Spica mutica, cœrulescente, glabra.

I. Spica exigua.

\section{Triticum amyleum.}

Giaucescens. Spica compressa, adscendente; spiculis arcte inbricatis; gluma in nucronem latiusculum prolon- 
gata ; carina compressa, valde prominente, curvata, lateribus convexiusculis; seminibus triquetris, lon ris, acutis, gibbis, opacis; culmo solido.

A. Spica aristata, alba, glabra; glumae mucrone in. curvo.

B. Spica submutica, alba, glabra; seminibus turgidis.

C. Spica aristata, alba, villosa.

D. Spica aristata, atrata, villosa; seminibus obscuris.

E. Spica aristata, alba, glabra, ramosa.

\section{Triticum monococcum.}

Flavescens. Spica compressa, arcte imbricata; gluma inæqualiter bidentata, bicrenata, subnervosa; valvula interna lineari, obtusa; seminibus oblique triquetris, (ory2oideis) subpellucidis.

A. Spica aristata, rufa, glabriuscula.

8. Triticum venulosum.

Spica compressa, arcte imbricata ; gluma unidentata, bicrenata, ad carinain venosa; valyula interna lata; seminibus......

A. Spica aristata, rufa, glabra. 
SECTIO SECUNDA.

Spelice.

Seminibus trigonis, matu. ris valvatis; rachi fragili; gluma guadriflora; flori= bus duobus (velunico) fertis. iibus.

\section{SECONDE SECTION.}

\section{Epeautres.}

Graincs trigones, tombant enveloppées à leur maturité par les vaives de la glumelle; glume quadriffore dont deux, rarement une seule, fertiles.

\section{TRITIC U M S P E T A.}

Spica subtetragona, inclinata; spiculis laxe imbricatis; gluma truncata; carina subcompressa, rectiuscula, lateribus planiusculis; seminibus triquetris, longis, acutis, opacis; culmo cavo.

Epi presque tétragone, incliné; épillets làchement imbriqués; glume tronquée; carène peu comprimée, presque droite, côtés planiuscules; graines triquètres, longues, aigues, opaques; chaume creux.

Obs. 1. Cette espèce, presque exclusivement cultivéc dans la partie allemande de la Suisse, offre des caractères très-tranchés; ses épis sont toujours lâchies, presque tétragones, légèrement penchés à la maturité par une légère ct rigide arcuation du chaume, immédiatement sous l'épi; ses glumes, tronquées au sommet, sont terminées par un mucrone obtus, prolongement de la nervure de la carène; ses graines triquètres, longues, pointues. La valve externe de la glumelle des deux fleurs latérales de chaque épillet, seules fertiles, sont terminées à l'état par- 
fait, comme dans tous les blés, par une arète ou barbe dure, qui avorte très-souvent, et alor's cette valve est quelquefois bidentée et d'autre fois presque tronquée.

Obs. 2. L'écartement des épillets est très-variable dans cette espèce surtout; car depuis l'état que Mr. Host a figuré sous le nom de Trit. Zea, et qui offre l'extrême de leur rapprochement, jusqu'à celui où les articulations du rachis sont aussi grandes que les épillets, dernier dégré de leur écartement, il se trouve des nuances infinies, qui cependant laissent toujours à l'épi de cette cspèce, toujours mince et alongé, un certain aspect qui lui eat propre.

Obs. 3. Cette espèce offre, comme toutes les autres, des passages de l'état glabre à l'état velouté des épis, mais l'état glabre est presque général, ce qui m'a fait croire quelque temps que l'épeantre était toujours glabre.

Obs. 4. Toutes les parties de cette plante, particulièrement le haut du chaume et l'épi, sont couvertes, vers l'époque de sa fleuraison, d'une trèslégère efleurescence pruincuse, qui rarement se trouve dans l'état de maturité. Jusqu'alors toutes les variétés, établies sur les conleurs, ne peuvent être distinguées; mais au commencement de la maturation on voit la plante passer du verd glauque au paille, au roux, au violet, au bleu-gris. Souvent le chaume est violet et l'épi entièrement blane, souvent même il n'a cette teinte violette que d'un seul 
côté du chaume, et l'autre est blanc. Quelque fois aussi la légère effleurescence pruineuse, dont l'épi est particulièrement couvert pendant la fleuraison, se conserve et donne aux épis roux une teinte de couleur de chair très-agréable.

\section{T'Rit. SPELTA.}

A. Spica aristata, alba, glabra.

A. Epi barbu, blanc et glabre.

ZeA sive spelta J. Bauh. Hist. 2.p. 412. (mauvaise fig., très-bonne descript.) - Rai Hist. 2. p. $1242 . n .01$.

Trit. spelta Lin. Hort. ups. 21. - Spec. 1. p. 127. n. ${ }^{0}$ 5.-DeC. Fl. fr. 3. n. ${ }^{0}$ 1658. p. 82. var.a. - Dum. Cours. Bot. cult. Ed. 2. vol. 2. p. 110. n.0 3. - Gard. Agrost. 1. p. 339. - Gilliaboz Portr. de plant. dessinées 1. T. 64. (tr. bien.) - Gouan Fl. Monsp. 128. - Flost. Gram. Austr. 3. T. 30. $\left(^{*}\right)$ - Kern. whon. Pfunz. 23. Heft. T. 290. f. 1. - Loisel Fl. gall. 1.p. 71. - Pers. Syn. 1. p. 109. n.0 4. - Roem. et Schult. Sy'st. 2.p.767. var. a. aa. n. ${ }^{0}$ 21. - Sut. Fll. helv. 1. p. 75. n. ${ }^{0}$ 5. - Tration. Tabuel. T. 369. (dapres Roem. et Schult. Syst.) -

( ${ }^{*}$ Lcs Tr. Spclta T. 30. et Zea T. 29. de Mr. Host ne sont certaintment pas deux espèces distinctes, et pas même deux variétés; dans son Zea les épillets sont un peu plus distants que dans son Spelta, mais cet ćcartement varie beaucoup, jamais cependant au point de pouvoir confondre aucune des variétés du T. Spelta L. avec le $\mathrm{T}$.'amyleum. 
Till. Hist. 2. p. 158.n. no 6. - Willd. Spec. 1. p. 478. n.0.6. Enum. 1. p. 134.

Triticum florilus quaternis, duobus fecundis. glumis adharentibus Hall. Hist. $n .{ }^{0} 1424$.

Trirrcum locustis trifloris, callycibus iruncatis, mucronatis, calycibus cartilagineis semen continentibus Hall. Nov. Comm. 5. p. 17. (dans leque? il a confondu le $T$. amyleum.)

Trit. zea Host. Grum. Aust. 3. T. 29. (*) Ræm. et Schult. Syst. 2. p. 766. (**)

Trit. Dicoccum Schrenk. bav. 339. (d'apre's Ricem. et Schult. Sy'st. 2. p. 766.)

Fig. Kern. okon. P1. 23. Heft T. 290. f. 1.

Gilliab. Port. de pl. dessin. 1, T. 64.

Host. Gram. Aust. 3. T. 29. et 30.

Ser. Herb. cer. 5. A. (matur.)

Franc. Epeautre blanc, bạbu et glabre. E barbu à épi blanc. DeC.

Allem. Spclze, Korn, Denkel, Dünkel, Spelt, Dinkelkorn, Zweikorn, Dinkelweizen, Corallenweizen.

$\left.{ }^{*}\right)$ Voyez la note précédente du $T$. Speltc.

$\left({ }^{*}\right)$ Le Trit. Zea Host. Gram., n'appartient pas a la section des blés à graines nues, comme l'ont rapporté MM. Rœm. et Schult. Syst. 2. p. 766., mais à leur section B. bien saisie, de : Semen matzurum valvulis calycinis corticatum, rachis fragilis. 
Angt. Spelt wheat, Germann wheat, Spelt. Crone. Espag. Escanda.

5. TRIT. SPELtA.

B. Spica aristata, alba, velutina.

B. Epi barbu, blanc, velouté.

FIG. Ser. Herb. cer. 5. B. (compr.)

Obs. Ne diffère de la var. précédente que par les poils mols, qui recouvrent l'épi.

Francs. Epeautre barbu, blanc et velouté.

\section{Trit. spelta.}

C. Spica aristata, rufa, glabra.

C. Epi barbu, roux et glabre.

TRYT. spelta b. épeautre barbu da épi rouge. DeC. Fl. fr. 3. $n .{ }^{0} 1658$. p. 82 .

TRIT. SPELTA a. b. spica barbisque rufescentibus. Rom. et Schult. Syst. 2. p. 767. n., 21.

Fig. Ser. Herb. cer, 5. C. (matur.)

Franc. Epeautre barbu, roux et glabre.

5. Trit. Spelta.

D. Spica mutica, alba, glabra.

D. Epi sans barbes, blanc et glabre. 
ZeA spica mutica dicoccos vel major. Moris. Hist. 3. p. 204. S. 8. T. 6. fig. 1. (Il réunit dans la description les variétés barliues et sans barbes.)

Trit. sperva (*) Kern. œhon. P f. 23. Heft. T. 290. f. 2. 3. - Gilliab. Portr. de pl. dessin. 1. T.63. var. a. - J. Gessner Tabul. phytogr. Tab. 7.f.94. n. ${ }^{1}$ 1. - Tr. Spelta. c. Eperutre sans barbes à épi blanc. DeC. Fl. fr. 3. n. ${ }^{0}$ 1658. p. 82. -

FIG. Moris. Hist. 3. p. 204. S. 8. T. 6. f. 1.

Kern. okon. Pfl. Heft 23. T. 290. f. 2.3.

Gilliab. Portr. de pl. dessin. 1. T. 63.

J. Gessn. 'Tabul. phyı. T. †. f. 94. n. ${ }^{0} 1$.

Ser. Herb. cer. 5. D. (mat. et compr.)

Franc. Epeautre sans barbes, blanc et glabre.

5. Trit. Spelta.

E. Spica mutica, rufa, glabra. E. Epi sans barbes, roux et glabre.

Trit. SPELTa $d$. Epeautre sans barbes à épi rouge. DeC. Fl. fr. 3. $n .^{0} 1658 . p .32$.

TRIT. SPELta a. spica rufescente subgracili, glumis laxis et muticis. Roen. et Schult. Syst. 2 , p. $76 \%$. (**)

$\left(^{*}\right)$ Cette variété est très-fréquemment cultivée dans le canton de Berne, surtout dans la plaine, elle murit, dit-on, quinze jours plutot que le Tr. Spelia. Spica mutica, riefa, glabra.

( $\left.^{* 4}\right)$ Cette variété est plus particulièrement cultivée sur les basses montagnes; elle murit, au rapport des babitants 
Fig. Ser. Herb. cer. 5. E. (matur.)

Franc. Epeautre sans barbes, roux et glabre.

5. TRit. Spelta.

F. Spica mutica, rufa, velutina.

F. Epi sans barbes, roux et velouté.

FIG. Ser. Herb. cer. 5. F. (matur.)

Obs. Je n'ai rencontré de variétés velues que cette année; j'ignore si on peut l'attribuer à la sécheresse qui a eu lieu, ou si je ne les avais pas remarquées auparavant.

Franc. Epeautre sans barbes, roux et velouté.

5. Trit. spelta.

G. Spica mutica, violacea, glabra.

G. Epi sans barbes, violâtre et glabre. .

Obs. Cette variété à chaume et épi d'un violet vif sur le frais, acquiert un violet sale à la maturité, époque à laquelle les épillets deviennent trèsbruns.

Franc. Epcautre sans barbes, violet et glabre.

du pays, quinze jours plus tard que le Tr. Spelix. Spica mutica, alba, glabra, mais parait plus robuste, et produit plus de farine; iı assurent aus si que cultivée dans les terrains humides des plaines, cette variété ronsse de-vient blanche, c'est pour cela qu'ils s'en procurent de temps en temps des montagnes pour faire les semis. 
5. Trit. SPELTA.

H. Spica mutica, corulescente, glabra.

H. Epi sans barbes, bleuàtre et glabre.

Obs. On cultivait beaucoup, il y a unc dixaine d'années, dans ce canton celte variété; mais on a trouvé qu'elle rendait peu de farine, et son arrêt a été prononcé par les meuniers, qui ne l'achetaient plus dans les marchés. Elle est devenue si rare, qu’il m'a été absolument impossible de m'en procurer un seul épi ces deux dernières années, quoique je l'aie bien cherchée. Elle avait le haut du chaume d'un jolis gris d'améthyste, et l'épi d'un gris sale. - Je prie les cultivateurs qui l'auraient encore, de m'en envoyer quelques cents épis murs bien conservés.

Froanc. Epcautre sans barbes gris-bleu.

\section{Trit. SPELta.}

I. Spica exigua.

I. Epi apauvri.

Frg. Ser. Herb. cer. 5. I. (matur.)

Obs. Cette variété n'est due qu'à l'épuisement du sol, clle ne se trouve que dans les terrains mal cultivés et au bord des chemins; je l'ai semce dans des terrains gras, et ellc a repris la forme et la longueur des épis moyens.

Franc. Epeaulre sans barbes apauvri. 


\section{TRITICUM AMYLEUM.}

Glaucescens. Spica compressa, ascendente; spicutis arcte imbricatis; gluma in mucronem latiusculum prolongata; $\left({ }^{*}\right)$ carina compressa, valde prominente, curvata, lateribus convexiusculis; seminibus triquetris, longis, acutis, gibbis, opacis; culmo solido.

Glaucescent. Epi comprimé, ascendent; épillets densément imbrisqués; glume insensiblement terminée par un large mucrone; $\left({ }^{*}\right)$ carène comprimée, très-saillante et arquée, côtés convexiuscules; graines triquètres, longues, pointues, bossues et opjaques; chaume plein.

Obs. La teinte glauque de toutes les parties de la plante, qui la fait reconnaitre de loin, la forme de l'épi ascendant, toujours manifestement comprimé, et très-dense dans cette espèce; son mucrone large, obtus, souvent incliné, sa carène fortement comprimée, à côtés convexiuscules, ses żaines triquètres, bossues vers le hile, et son rachis très-fragile caractèrisent parfaitement cette espèce, que les auteurs anciens ont bien connue.

(*) J'ai aussi retrouvé dans cette espèce ce que j’ai noté ailleurs; c'est que: lorsque les barbes sont irès_courtes ou nulles, le mucrone diminue au point de devenir presque nul. Ainsi le mucrone est toujours long ou court en raison directe de la longueur ou de de la brièveté des barbes. 
6. Trit. amyleum.

A. Spica aristata, alba, glabra; glumce mucrone incurvo.

A. Epi barbu, blanc, glabre; mucrone de la glume courbé.

Zea verna J. Baih. Hist. 2.p. 413. (figure en bois, bonne.) - Rai Hist. 2. p. 1243. n.0 1.

Zea amylea seu olyra $C$. Bauk. Theat. 412. T. 414 .

Zea amylea vel zeocriton $C$. Bauh. Pin. 22.

TRITICUM floribus quaternis, duobus fecundis, glumis adhorentibus. Hall. Hist. n.0 1424. (confondu avec le T. Spelta.)

TrIrIcum locustis trifloris, calycibus truncatis, mucronatis, calycibus cartilagineis semen continentibus. Hall. Nov. Comm. 5.p. 17. n.0 13. (aver le Tr. Spelia.)

Trit. spelta Willd. Spec. 1. p. 4T8. n.0 6. (dont une partie de la synonymie appartient au $T r$. ainyleum). - Roem. Schult. Syst. 2. p. 767. (confondu avec le Tr. Spelta.)

Trit. Spelta var. E., épeautre serré. DeC. Fl. fr. 3. n. ${ }^{0}$ 1658. p. 82.

Trit. monococcum andus Dum. de Cours. Bot. cult. éd. 2. vol. 2. p. 110. $n .05$.

Trit. cienfuegos. Gluma disperma : valuilis 
subbidentis, dentiörus unguiculatis, semine lumido: Lagasca, Gén. et Spec. n.0 83. (d'aprés Mrs. Riocm. et Schult. Syst. 2. p. 767. n.0 19. qui l"ont aussi adopté comme espéce.) (*)

Trit. zeA, T. zEA WÜRTENBERGICUMI, T. DICOCCUM, T. DICOCCUMI ROMIANUM, T. DICOCCUM PERsicum Fellenb. Inst. de Hofw.

FIG. J. Bauh. Hist. 2. p. 413. (en bois.)

C. Bauh. Theat. 'T. 414.

Ser. Herb. cer. 6. A. (matur. et compr.)

Obs. 1. C'est particulièrement de cette espèce, cultivée dans quelques vallées des Alpes, et plus en grand dans l'Aargovie, que les habitans de ce' canton font leur bel amidon.

Obs. 2. Cette plante, qui réussit dans tous les terrains, est cultivée plutot en céréale de printemps qu'en céréale d'automne. Scmée le 26. Scptembre 1816 à Interlacken, par Mr. de Haller, il en a fait la récolte le 6 Août. - Je l'ai aussi seméc en automne, et elle a parfaitementáussi. Je l'ai vuc dans des tourbières, dans des prés marícageux, ou il n' $Y$ avait auparavant que de l'Arundo phragmiles, et dans les terrains les plus secs.

$\left(^{*}\right)$ Il me parait aussi très-probable que le T. Bauhini Lagasca (d'après et avec MM. Rom. et Schult. Syst. 2. p. 767. n. 20.) n'est qu'une vảriétcé d̀ épi velouté du Tr. amyleum. 
Obs. 3. Je crois que cette espèce, dont la farine est très-blanche, produit un pain qui se desséche très-facilement, mais comme elle est extrềmement rustique, et qu'elle mérite surtout d'ètre cultivée dans les vallées alpines, où elle réussit fort bien, on pourrait en mêler la farine avec une petite partie de farine de seigle.

Obs. 4. Les épis de cette espèce ne varient pas moins de longueur et de largeur que ceux des autres espèces. Des deux épis, qui sont dans mon Herbarium cereale, l'un offie l'état moyen, et l'auire le plus petit; mais ce dernier, récolté un peu avant sa maturité, conserve encore en partie sa teinte bleuàtre.

Franc. Blé amidonier. - Grande épeautre. Dum. de Cours. - Blé de Jérusalem.

Allem. Amer, Ämer, Ämerkorn, Immer, romanischer Sommerweizen, (et dans l'Oberland bernois) Jerusalem-Korn.

TRIT. AMYLELim.

B. Spica submutica, alba, glabra; seminibus turm gidis.

B. Epi à barbes courtes, blanc, glabre et à grossẹ graines.

Zea amylea Moris. Hist, 3. p. 205. S, 8. T. 6. f. 3 . 
Gramen spicae Brizae majus Rai Hist.p. $120 \%$.

Trir. zea hybernum Fell. Inst. d'agricult.

FIG. Moris. Hist. p. 205. S. 8. T. 6. f. 3.

Obs. Cette variété à gros épis, à très-gros grains, manifestement bossus, se trouve rarement mêléc avec la précédente; ses barbes sont courtes et ordinairement avortées à la base de l'épi, alor's la valve externe de la glumelle n'est plus que Jonguement mucronée. La glume a conséquemment son mucrone en proportion, et il est beaucoup plus obtus que dans la variété précédente.

Franc. Blé amidonier à courtes barbes.

Allem. Aegyptischer Winterweizen.

6. 'Trit. AMYLEUM.

C. Spica aristata, alba, villosa.

C. Epi barbu, blanc, velouté.

Trit. dicoccum russicum Fell. Inst. d'agricult.

Obs. 1. Céte variété ne se distingue de la première que par son épi velu; la carène de la glume est la dernière partie qui perde les poils, et on en rencontre quelques-uns vers le mucrone de ma var. A.

Obs. 2. Extrêmement rare dans les champs de la Suisse.

Franc. Blé amidonier variété à épi velouté Allem. Russischer Mehldinkel.

6. TRIV. 
6. Trit. amyleum (T. atratum.)

D. Spica aristata, atrata, villosa; seminibus obscuris.

D. Epi barbu, noirâtre, velu; graines brunâtres.

Trit. atratum, spiculis bifloris, imbricatis, pubescentibus aristatis, racheos internodiis ad oras pilosis, foliis basi ciliatis. Host. Gram. Aust. 4. T. 8. - Roem. et Schult. Syst. 2. p. 766. (*)

Fig. Host. Gram. Aust. 4. T. 8.

Ser. Herb. cer. 6. D.

Obs. 1. Aucun caractère ne différencie cette espèce de Mr. Host d'avec les trois autres variétés du Trit.anyleum. Le velu, qui couvre toutes les parties de l'épi exposées à la lumière, la ligne noire, qui borde la glume, quelquefois un peu plus d'applatissement de l'épi, ne sont là que des caractères de simples sous-variétés.

Obs. 2. Cette variété n'est cultivée que par quelques amateurs, et la firine noire, que je présume qu'elle produit, à cause de la teinte sale de sa graine, ne la feront surement pas rechercher.

Franç. Blé amidonier noirâtre.

6. TRIT. AMYLEUM.

E. Spica aristata, alba, glabra, ramosa.

F. Epi barbu, blanc, glabre et rameux.

(*) Cette espèce appartient a la section des épeautres (Spelta), et non aे celle cies froments (Frumenta), comme l'ont rapporté MM. Rom. et Schult. Syst. 
Trit. spelta subcompositum DeB. (Epi mur.)

C'est à Mr. de Buren de Vaumarcus, qui a déjà fait de nombreux essais sur les céréales, que je suis redevable de cette belle variété, dont l'épi est rameux dans sa moitié inférieure. Les épillets du sommet sont conformés comme dans les variétés $A B$, mais un peu plus écartés.

Ce singulier état m'a offert l'occasion de voir que le rachis de chaque épillet, extrèmement court dans l'état naturel, s'alonge dans les épillets rameux, ce qui donne aux fleurs centrales, qui avortent ordinairement, la faculté de se développer, n'éprouvant aucune pression qui les gêne. Les deux fleurs latérales sont appliquées contre les deux valves de la glume, mais la $3 .^{\mathrm{e}}$, $4 .^{\circ}$ et même souvent 5. fleur de l'épillet, lesquelles ont toujours les deux valves de leur glumelle, sont encore munies d'une glume unie ou rarement bivalve, semblable à celle des iépillets régulièrement conformés.

\section{TRITICUM MONOCOCCUM.}

Fluvescens. Spica compressa, arcte imbricata; gluma inoqualiter bidentata, bicrenata, subnervosa; valvula interna lineari, obtusa; seminibus oblique triquetris (oryzoüdeis) subpellucidis.

Jaunâtre. Epi comprimé, densément imbriqué; şlume inégalement bidentée ct bicrénéc, faiblement nervée; valve interne de la glumelle linéaire, obtusc ; graines obliquement triquètres, (oryzoïdes) demi transparentes. 
7. Trit. Mono coccum.

A. Spica aristata, rufa, glabriuscula.

A. Epi barbu, roux, presque glabre.

ZEA MONococcos BRIZA quibusdam J. Bauh. Hist. 2. p. 413. (mauvaise fig., mais bonne description et synonymie.) - Rai Hist. 2. p. 1242. n. ${ }^{0} 2$.

Zea briza dicta s. monococcos germanica $C$. Bauh. Theat. p. 413. - Moris. Hist. 3. p. 225. S. 8. T. 6. f. 2.

Tríricum Spica disticha, floribus ternis, fertili unico, glumis tridentatis, florali aristato. Hall. Hist. n. 01425.

Triticum Spica disticha, locustae floribus ter. nis, binis aristatis. Hall. Nov. Com. vol. 5. p.18. n. ${ }^{0}$ 15. (T. 1.f. 11. 17?)

Trit. monococcum Lin. Spec. 1.p. 127. - DeC. Fl. fr. 3. n. 0 1659. a. p. S3. - Dum. de Cours. Bot. cult. éd. 2. vol. 2. p. 110. - Gaud. Agrost. 1. p. 340. - Gouan Fl. Monsp. p. 128. n. ${ }^{0}$ 4. - Hoffm. Deutschl. Fl.p. 43. n. 1. - Host. Gram. Aust. 3. T. 32. - Lagasca Gen. et Spec. n.0 82. - Loisel Fl. gall. 1. p. 71. - Pers. Syn. 1.p. 109. n. ${ }^{0} 5 .-$ Roem. et Schult. Syst. 2. p. 766. n.0 18. - Sut. Fl. helv. 1. p. 75. n.0 6. - Vill. Hist. 2. p. 159. n. 7. - Willd. Spec. 1. p. 479. n.07. - Enum.1.p. 134. $n .06$.

Fig. Host. Gram. 3. T. 32.

Ser. Herb. cer. 7. A. (mat. et compr.) 
Obs. 1. Cette espèce offie une foule de caractères solides. D'abord la teinte jaune de toute la plante et ses petits épis la font reconnaitre de loin; puis son épi très-dense et très-comprimé; sa glume inégalement et aigument bimucronée, et plus en arvière, munie de deux crénelures membraneuses, qui terminent supérieurement chaque bord de la glume. En outre la valve interne de la glumelle est linéaire et obtuse, une seule des quatre fleur's porte une graine transparente, comprimée, triangulaire, et qui, si elle était blanche, ressemblerait au riz.

Obs. 2. Cette espèce est très-peu productive, quant à la quantité des graines et à leur volume, car elles sont les plus petiles du genre; mais elle offre d'autres avantages notés par Villars dans son Histoire des plantes du Dauphiné, 2. p. 159. a. "Cette espèce, (Triticum monococcum) se sème en automne, même avant les blés, et murit plus tard, de sorte qu'il est de tous les blés celui qui reste le plus longtems en terre. (*) On l'a souvent vu y passer l'année entière et mème plus dans les montagnes; son épi est plus mince, plus grèle et luisant; il n'a qu'un seul rang de grains, ce qui distingue aisément cette plante; sa paille talle beaucoup, il faut le semer très-clair, et le plus mauvais sol lui suffit, s'il n'est pas trop humide; il pousse des brins de feuilles en automme, qui ont

(*) Villars aura surement été induit en erreur; il aura cru qu'on le sem.it en automne (tandis que c'est au printems), et aura été surpris de le voir cncore trèsvert après la récolte des autres blés. 
l'air d'un Gramen languissant, tandis que sa racine se fortifie et donne jusqu'à vingt-cinq ou trente tiges au printemps, qui sont si fermes, si dures que les animaux n'en mangent pas; on l'employe pour couvrir les maisons en chaume, à quoi elle est trèspropre par sa fermeté et par son usage; son grain est moins sujet au noir (charbon, Uredocarbo DeC.) que celui du froment ordinaire, on s'en sert aux nèmes usages que l'ćpeautre; mais il est de meilleure qualité; son grain fait un pain léger, quoique brun, mais on le réserve pour faire du gruau de la première qualité. "

Obs. 3. Cette espèce est asscz cultivée ça et là en Suisse, particulièrement entre Thun et Belp, rive gauche de l'Aar. J'en ai trouvé, mais assez rarement, des épis attaqués du charbon.

Franc. Blé locular ; petit épeautre. Froment locar. Froment monocoque. Froment uniloculaire.

Allem. Einkörniger Weizen. Sanct-Peters Korn. Einkorn. Blicken.

Espag. Esprilla, Carraon, Escanna.

\section{TRITICUM VENULOSUM.}

Spica compressa, arcte imbricata; gluma unidentata, bicrenata, ad carinam veriosa; valvula interna lata; seminibus. . . . . .

Epi comprimé, densément imbriqué ; glume unidentée et bicrénée, transversalement et fréquem- 
ment veinée vers la carène; valve interne de la glumelle large ;raines. ......

8. Trit. venulosum.

A. Spica aristata, rufa, glabra. A. Epi barbu, roux et glabre.

Obs. L'impossibilité de rapporter à aucune autre espèce, que je connaisse, une plante récoltée en Egypte, que Mr. le Professeur Desfontaines m'a cnvoyée sans étiquette, et qui a beaucoup de rapports avec le Trit. monococcum, m’a engagé à l'établir comme espèce, pour aumoins fixer l'attention, et voir si l'on ne trouvera pas dans la graine quelques caractères, qui puissent appuyer ceux que je vais signaler. Ia plante n'etant qu'en fleur, je n'ai pu acquérir l'entière conviction qu'elle appartient à la section des Epeautres, mais son extrème ressemblance avec le Trit. monococcum me le fait croire. L'épi est un peu plus large que celui de ce dernier, asse $z$ court; la glume est terminée par un seul mucrone, ressemblant au plus grand du Trit. monococcum, et comme lui accompagnée sur les parties latérales de deux larges crénelures : cette glume est relevée sur la carène de petites et fréquentes veines obliques, anastomosées; caractère singulier, qui lui a mérité son nom; la valve interne de la glumelle au licu d'être linćaire, comme dans le Trit. monococcum, est large, et de la même forme que l'externe. D'aillcur's l'ćpi est lustré, garni de quelques poils vers la partic supérieure de la carène, longuement aristé et le chaume est plein.

Franc. Blé veiné. 


\section{S E C A L E.}

Lin. Toumef. Hall. etc.

S. Floribus spicatis; rachi flexuosa; compressa, articulata; spiculis alternis in summis articulis sessilibus; gluma bivalsi, biflora, in aristam brevem elongata, carinaque ciliis rigidis fumbriata; glumella bivalvi; valvula exteriori cymbiformi; seminibus ellipsoïdeis, apice truncatis.

$$
S E I G \cdot L E \text {. }
$$

Flcurs en épi ; rachis flexueux, comprimé, articulé; épillets alternes, naissants du sommet de chaque articulation; glume bivalve, étroite, hiflore, insensiblement terminée par une arête courte, et bordée ainsi que la carène de cils roides; glumelle bivalve; valve externe naviculaire, graines ellipsoïdes, tronquées au sommet.

\section{S E C A L E E R E L E.}

A. Spica simplici.

A. Epi simple.

Secale J.Bauh. Hist. 2,p. 416. - Tournef. Inst. 3. T. 294.

Secale hybernum vet masus $C$. Bauh. Theat. p. 425. 
Secale cereale Lin. Hort.ups. 22. - Spec. 1. p. 124. - DeC. Fl.fr. 3. n. ${ }^{0}$ 1573. p. 85. - Dum. de Cours. Bol. cult. ed. 2. vol. 2. p. 113. - Gaud. Agrost. 1. p. 143. - J. Gessn: Tabul. phyt. T. 7. f. 92. n. 1. - Gouan IT. Nionsp. 127. - Host. Gram. 2. T. 48. - Loisel. Fl. gall. 1.p. 68. - Pers. Sy'n. 1. p. 10S. - Rocm. et Schutt. Sy'st. 2. p. T73. - Sut. Fl. helv. 1. p. 74. - Vill. Hist. 2. p. 168. -Willd Spec. 1.p. 4T1. Enum. 1. p. 132.

Secale glumarum cilis scabris Hall. Hist. $n .0$ 1421.

SECALE glumis floralibus glabris, ora denliculata. Hall. Nov. Com. 6. p. 11. n.0 22.

FIG Tournef. Inst. T. 294.

Host. Gram. Aust. 2. T. 48 .

Exs. Ser. Herb. cer. 9. A. (mat.)

Obs. 1. Le Seigle ne varie pas moins que les blés, quant à la hauteur de son chaume et à la longueur de son épi; malgré que les terres maigres puissent lui suffire, il réussit beaucoup mieux dans les terres fumées. Dans ces dernières son épi atteint quelqucfois cinq et six pouces de longueur, tandis que dans les terrains maigres et picrreux il n'a que quatre à cinq épillets dè chaque côté.

Obs. 2. Malgré toutes les recherches que j’ai pu faire jusqu'à présent dans le seigle dit de printemps, il m’a été impossible d'y découvrir aucun 
caractère de variété, ni dans les parties de la fleur, ni dans la graine.

Obs. 3. Le seigle résiste très-bien dans les climats froids et forme en grande partie le pain du pauvre, qu'il rend très-nourrissant et cn même temps très-lourd par la grande quantité de matière féculente, et le peu de gluten qu'il contient. Mêlé en petite proportion avec la farine des blés en général, il donne du moellcux au pain, qui se desséche moins vite. La paille est surtout cmployée pour lier les gerbes, et quoique mince elle offre beaucoup de ténacité, ce qui empêche les seigles d'ètre versés aussi facilement que les blés par les pluies et les vents.

Franc. Seigle commun.

Allem. Gemeiner Roggen.

9. Sec. cereale.

B. Spica ramosa.

B. Epi rameux.

Secale spicis ramosis Tenzel. Hall. Nov. Com. 6. p. 13.

Secale cereale compositum DeC. Fl. fr. 3. n.0 1672. p. 88 .

Obs. Je n'ai jamais trouvé la variété du seigle à épi double, mais plusieurs personnes m'ont affirmé en avoir vu dans les champs de la Suisse, et Mr. le curé Müller m’en a envoyé un bel exempl. Lcs 
deux épis partent du sommet du chaume, qui n'est pas plus volumineux qu'à l'ordinaire; ils ont absolument d'ailleurs la conformation des épis ordinaires du seigle.

Franc. Seigle commun ramcux.

\section{H O R D E U M.}

Floribus spicatis ; rachi flexuosa, articulata; spiculis unifloris, ternis ad racheos apicem in verticillum dimidiatum congestis; gluma bisalvi, lineari, planiuscula; glumella bivalsi; valvula florum fertilium externa aristata, quinquenerrata, interna binervata; mutica, seminibus orödeis, sulcatis.

\section{$O R \quad G \quad E$.}

Fleurs en épi; rachis flexueux, articulé; épillets uniflores, demi-verticillé-ternés au sommet de chaque articulation; glume bivalve, linéaire, planiuscule; glumelle bivalve, valve externe des fleurs fertiles aristée, quinquénervée; valve interne binervée et mutique; graines ovoüdes, sillonnées.

Autant j'ai fait d'efforts pour réunir dans le genre Trilicum, autant jai cherché à diviser dans le genre Hordeum, sans avoir pu trouver dans ce dernier. le moindre caractère de nouvelles espèces. 
Ce genre est formć de deux sections, si tranchées en apparence, que Mi. Palisot de Beauvois a cru devoir en faire deux genres; mais, quoique très-différentes au premier coup d'œil, les orges offrent cependant les mèmes caractères fondamentaux.

'Toutes les orges ont les épillets uniflores, la glume bivalve, linéaire et en alêne. Trois flcurs disposées en demi-verticille, naissent alternativement du sommet de chaque articulation du rachis. Ces trois flurs (ou épillcts) sont tontes fertiles et sessiles dans les Orges Hexastiques (Hexasticha), tandis que dans la seconde section des Orges distiques (Disticha), des trois fleurs d'un demi-verticille, la centrale seule est fertile et sessile, et les deux latéralcs mâles, conséquemment stériles, et pédicellćes. Les deux valves de la Glume, dans les fleurs de toutes les orges, sont linéaires, légèrement velues à leur face externe, et insensiblcment terminées en alène. La glurnelle des fleurs fertiles et stériles est bivalve; mais dans les fleurs fertiles la valve externe est. à cinq norvures (une centrale, qui va former la nervure de l'arète, et les deux latérales de chaque côté, qui s'anastomosent, au sommet de cette valve externe de la glumelle, pour former les bords de l'arête, qui est hérissée de poils courts, roldes et obliques). La valve interne de la glumolle des fleur's fertiles cst large, sans arête, binervée et plus petite que l'externe, qui l'embrasse. par les bords. Dans les Reurs máles et pédicellées des orges de la seconde seclion (Disticha) les deux. 
valves de la glumelle sont lancéolé-linéaires et obtuses. - La glumellule est aussi bivalve. Ces valves sont placées latéralement vers la jonction des bords de la glumelle : ce sont de petits corps paléacés, bordés de poils fins et nombreux. Ces deux valves de la glumellule sont difficiles à trouver dans les orges à graines enveloppées, à cause de l'étroite application des valves sur la graine; mais elles sont très-visibles dans les Orges à graines nues.

Ainsi done les épillets uniflores, tous demi-verticilé-ternés; la glume bivalve, linéaire; la valve externe de la glumelle quinquénervée (dans les fleurs fertiles des orges des deux sections), et l'extrême ressemblance des graines, tout me semble ne pouvoir permettre de diviser le genre Hordeum de Linné qu'en deux sections, mais non en deux genres.

\section{TABLEAU DES ESPĖCES ET DES VARIÉTÉS.}

\section{SE C TIO PRIM A}

HOR DEA HEXASTICHA

(Hordea. Palis. Beauv.)

Spiculis omnibus fertilibus, sessilibus.

10. Hordeum hexastichon.

Spica ellipsoïdea, densa, rigida, ascendente; spiculis divergentibus, æqualiter hexastichis; rachi brevi, rigida.

A. Spica hexasticha.

B. Spica abortiva, tetrasticha. 


\section{Hordeum vulgare.}

Spica cylindracea, laxa, elongata, nutante: spiculis inæequaliter hexastichis; rachi longa, flexili.
A. Seminibus vestitis; spica flavescente.
B. Seminibus vestitis ; spica nigricante.
C. Seminibus nudis ; spica flavescente.

\section{$\overline{(2+2}$ \\ S E C T I O S E C U N D A \\ HOR DEA D I S T I C H A. \\ (Zeocrita. Palis. Beauv.)}

Spicula media cujusque verticilli dimidiati sola fertili, lateralibus masculis, pedicellatis, rachique adpressis.

\section{Hordeum distichon.}

Spica compressa, lateribus parallelis; aristis erectis.

A. Seminibus vestitis; spica flexili, elongata; spiculis laxe, imbricatis.

B. Seminibus vestitis; spica rigida, brevi; spiculis dense imbricatis.

C. Seminibus nudis, inflatis; spica flexili, spiculis laxe imbricatis.

D. Spica mutica.

\section{Hordeum Zeocriton.} bus.

Spica compressa, pyramidali; aristis valde divergenti- 
SEC TIO PRIMA.

HORDEA HEXASTICHA.

(Hordea Pal. Beauv.)
SECTION PREMILRL. ORGES A SIX RANGS.

Spiculis omnibus fertilis Epillets de chaquedemi-verbus, sessilibus. ticille tous fertiles et sessiles.

\section{HORDELIM HEXASTICHON.}

Spica ellipsö̈dea, densa, rigida, ascendente; spiculis divergentibus, equaliter hexustichis; rachi brevi, rigida.

Epi ellipsoïde, dense, roide, et dressé; épillets divergents, sur six rangs régulièrement disposés; rachis court et roide.

10. Hord. hexastichon.

A. Spica hexasticha.

A. Epi à six rangs.

Hord, hexastichum pulchrum $J$. Bauh. Hist. 2. p. 429. cap. 14.

HordeUM fosculis omnibus hermaphroditis, aristatis; seminibus sexfariam aequaliter positis. Lin. Hort. ups. 23.

Hord. Hex.astichon Lin. Spec.1.p. 125. = DeC. Fl. fr. 3. n.0 1581. - Dum. de Cours. Bot. cult. éd. 2. vol. 2. p. 107. n. ${ }^{0}$ 3. - Gaud. Agrost. 1. p. 102. n. ${ }^{0}$ 2. - Host. Gram. 3. T. 35. - Loisel. Fl. gall. 2. p. 69. n. 2. - Pers. Syn. 1. p. 108. n.0 2. - 
Poir. Encycl. 6. p. 603.n.02. - Roin. et Schult. Syst. 2. p. 791. n. ${ }^{0}$ 3. - Sut. Fl. helv. 1. p. 74. zi.0 2. - Vill. Hist. 2. p. 1T2.n. ${ }^{0}$ 2. - Willd. Spec. 1. p. 473. n. ${ }^{0}$ 2. - Enum. 2. p. 1038. n. ${ }^{0}$ 3. -

Hordeum Spica polysticha, floribus omnibus hermaphroditis, longe arisiatis. Hall. Hist. n. ${ }^{0}$ 1534.

HoRdeUm (hexastichon) Floribus omnibus fertilibus; spica sexfariam sulcata. Hall. Nov. Com. 6. p. 3. ${ }^{0}{ }^{0}$ 18. T. 2.f. 22. 23. (trés-Lonnes.)

Fig. Hall. Nov. Com. 6. T. 2. f. 22.23. Host. Gram. 3. T. 35.

Exs. Ser. Herb. cer. 10. A. (matur. et compr.)

Obs. 1. Cette espèce, très-productive, est cultivée en céréale d'hiver, et en céréale de printemps. Seméc en automne, elle murit avant le blé, et peut, dans une année de disette, être très-utile par sa précocité. Elle réussit dans presque tous les terrains et se rencontre dans les vallées des Alpes, où toute culture céréale cesse. La longucur et le volume de l'épi varient beaucoup, selon le terrain, mais il est toujours ascendant, roide, et les graines sont régulièrement disposées sur six rangs.

Obs. 2. Les orges en général produisent une farine séche et un pain très-rude, elles entrent en partie dans le pain du pauvre, elles sont très-employées, dépourvues de leur glume et de leur péri- 


\section{4}

carpe et périsperme. (Voyez 4. ${ }^{\circ}$ partie de cette monogr.) Elles servent particulièrement, dans les pays où les glands manquent, à la nourriture des cochons, qui en sont très-friands.

Obs. 3. L'orge fermentée et mêlée avec le houblon produit la bière, boisson habituelle des pays privés de vignobles.

Obs. 4. La paille des Orrges est courte, séche et fragile, et ne sert guère que de litière.

Franc. Escourgeon, Orge à six rangs, O. à six côtés, O. anguleuse, 0 . d'hiver, O. carrée, soucrion.

Allem. Sechszeilige Gerste, Rollgerste, Stockgerste.

10. Hordeum heXastichon.

B. Spica abortiva, tetrastachia.

B. Epillets disposés sur six rangs, dont deux avortés.

Obs. 1. Cette variété est très-singulière; la fleur du rang central de chaque demi-verticille est complettement avortée, on en voit encore les rudimens, et ces quatre rangs n'ont rien perdu de leur symétrie, car ils sont tous placés à des distances égales.

Obs. 2. Je n'ai remarqué qu'une seule fois cet avortement du rang central de chaque demi-verticille, mais peut-être qu'en cherchant bien cette variété s'offrira plus souvent qu'on ne le croit.

Franc. Orge à six rangs, var. à quatre rangs (par avortement.) 


\section{HORDEUM VULGARE.}

Spica cylindracea, laxa, elongata, nutante; spiculis incequaliter hexastichis; rachi longa, flexili.

Epi cylindroïde, lâche, alongé et penché ; fleurs disposées sur six rängs xapprochés trois à trois; rachis long et flexible.

Obs. Cette espèce, quoique disposée sur six rangs, comme la précédente, en est certainement distincte. Les trois rangs de fleur's d'un demi-verticille sont très-rappochées, et l'épi conséquemment n'offre pas l'exacte symétrie de celui de l'Hord. hexastichon, dans lequel on remarque très-dificilement la ligne de séparation des demi-verticilles, tandis qu'elle est très-distincte dans l'Hord. vulgare. L'épi en outre a ses fleurs appliquées sur le rachis, et conséquemment ses arêtes parallèles, tandis que les fleurs et les arêtes sont très-divergentes dans l'Hord. hexastichon.

\section{Hordeum vulgare.}

A. Seminibus vestitis; spica flavescente.

A. Graines enveloppées; épi jaunâtre.

Hordeum polystachium $J$. Bauh. Hist. 2.p. 429. cap. 13. et p. 418. cap. 10. fg. 3.

Hordeum polystichum vernum Moris. Hist. 3. Sect. 8. T. 6.f. 3. (d'apres Willd.) 
HorDeUM flosculis omnilus hermaphroditis, se. minibus corticatis. Lin. Hort. ups. 22.

Hordeum vulgare Lin. Spec. 1.p. 125.-DeC. Fl. fr. 3. n. ${ }^{0}$ 16s0. var. a. - Dum. de Cours. Bot. cult. éd. 2. Tom. 2. p. 107. n.01. - Gaud. Agrost. 1. p. 100. ${ }^{0}{ }^{0}$ 1. - Host. Gram. 3. T. 34. - Loisel. Fl. gall. 1. p. 69. n.0 2.-Pers. Syn. 1. p. 108. n. 0 1. var. a. - Poir. Encycl. 6. p. 602. n. 1. - Roem. et Schult. Syst. 2. p. 791. n.0 1. - Sut. Fl. helv. 1. p. 74. n. ${ }^{0}$ 1. var. A. - Vill. Hist. 2. p. 172. n.0 1. - Willd. Spec. 1. p. 472. n.0 1. Enum. 2. p. 1037. n.0.2.

Hordeum spica subdisticha, calyce folioso setaceo; floribus omnibus hermaphroditis tonge aristatis Hall. Hist. n. ${ }^{0} 1533$.

HoRDEUM (polystachion) flosculis omnibus fertilibus, ordinibus indistinctis. Hall. Nov. Com. 6. p. 5. n. ${ }^{0}$ 19. T. 2. f. 18. 19. 20. (trés-bonnes.)

Fig. J. Bauh. Hist. 2. p. 418. f. 3.

Moris. Hist. 3. S. 8. T. 6. f. 3.

Hall. Nov. Com. 6. T. 2. f. 18. 19. 20.

Host. Gram. 3. T. 34.

Exs. Ser. Herb. cer. 11. A. (mat. et compr.)

Obs. Cette variété est souvent cultivée en céréale d'hiver, et alors elle sc récolte avant le blé ; d'autres fois en céréale de printemps, alors on ne la fauche qu'avec l'avoine. On la rencontre aussi jusqu'au fond des vallées alpines où les cérciales peuvent croìtre. 


\section{7}

Franc. Orge commune.

Allem. Wintergerste, Kerngerste.

\section{Hord: vulgare ( $H$, nigrum.)}

B. Seminibus vestitis; spica nigricante.

B. Graines enveloppćes; épi noirâtre.

Hord. vulgare nigrum Willd. Spec. 1. p. 472. n.0 1. var. $c$.

Hord. NIGRUM Willd. Enum. 2.p. 1037. n.0 2.Rom, et. Schult. Syst. 2. p. 791.n. ${ }^{0} 2$.

Obs. 1. Malgré tous mes efforts pour trouver dans cette variété à épi noir des caractères, qui aient pu m'engager à la séparer de l'Hord. vulgare, il m'a été impossible d'y voir d'autre différence que celle que présente la teinte noire et puineuse de l'épi à sa maturité; toutes les parties sont absolument conformées comme dans les autres variétés de cette espèce. Cette teinte noire n'est visible qu'à l'époque de la maturation; et malgré que je n'aie vu aucun passage de cette variété noire à la var. A. je suis persuadé qu'elle ne pourra jamais en être séparée. Elle n'est cultivée que par quelques amateurs, et probablement elle ne se répandra pas; seulement alors on pourrait juger des changemens qu'elle subirait.

Obs. 2. Malgré que l'on prétende que cette variété soit bisannuelle, je l'ai semée ce printemps, ct elle a bien réussi, mais l'année a été très-favorable. 


\section{8}

Franc. Orge commune à épi noir.

Allem. Russische Wintergerste. Fell. Inst. d'agr.

11. Hord. vulgare (H. coeleste.)

C. Seminibus nudis; spica flavescente.

C. Graines nues; épi jaunâtre.

HoRDEUM nudum sive gymnocrithon. J. Bauh. Hist. 2. p. 430. (bonne fig.)

HORDEUM flosculis omnibus hermaphroditis, seminibus decorticatis. Lin. Hort. ups. 23.

Hordeum vulgare coleste. (*) Lin. Spec. 1. p. 125. $n .^{0}$ 1. B. - DeC. Fl. fr. 3. n. ${ }^{0}$ 1680. var. $B$. - Loisel. Fl. gall. 1. p. 69. n. ${ }^{0}$ 1. B. - Pers. Syn. 1. p. 108. n. ${ }^{0}$ 1. B. - Poir. Encycl. 6. p. 602. n. ${ }^{0}$ 1. - Rom. et Schult. Syst. 2. p. 791. n. ${ }^{0}$ 1. B. Willd. Spec. 1. p. 472. n.0 1. B.

HoRdeum (polystichon) flosculis omnibus fertilibus, ordinibus indistinctis, varietas $B$. Hordeum cceleste. Lin. Hall. Nov. Com. 6. p. 6. T. 2. f. 21. (bonne fig.)

Fig. J. Bauh. Hist. 2. p. 430. (bonne fig. noire.) Hall. Nov. Com. 6. T. 2. f. 21. (id.)

(*) Le mot de caleste en marge de l'édition citée de Linné, est placé deux lignes trop haut, car l Hord. vulgare caleste $L$. n'est point synonyme de $r$ Hord. polystichon venum Bauh., c'est une faute typographique, 
Obs. Cette variété, peu cultivée, quoique méritant bien de l'ètre, mais cependant très-estimée de ceux qui la connaissent, et d'un prix beaucoup plus élevé que les autres orges, n'a pas le moindre caractère, qui puisse la faire regarder comme une espèce. La forme de l'épi, la disposition des fleurs, les glumes et glumelles, tout, excepté la nudité des graines, est parfaitement conforme aux caractères, qui signalent l'espèce.

Franc. Orge commune à graines nues. O. de Jérusalem. 0. de Sibérie. -

Allem. Nakte Gerste.

SECIIO SECUNDA. SECTION SECONDE.

HORDEA DISTICHA. ORGES A DEUX RANGS.

(Zeocrita Pal.Beauv.) (Zeocrites de Pal.Beauv.)

Spicula media crujusque Epillet central de chaque verticili dimidiati sola fer = demi-verticille seul fertile, tili, lateralibus sterilibus, Jes latéraux stériles, pédicel_ pedicellatis rachique adz lés et appliqués sur le rachis. pressis.

\section{HORDEUM DISTICHON.}

Spica compressa, lateralibus parallelis; aristis erectis, ascendentibus.

Epi comprimé, bords parallèles; arêtes ascendentes.

J'ai encore cherché dans cette section à établir plus d'espèces, sans pouroir les appuyer sur de 
bons caractères, ce qui m'a décidé à les laisser telles qu'elles ont été établies jusqu'à ce jour. Les var. A et $\mathbf{C}$ ont entr'elles la plus grande ressemblance, quant à la longueur de l'épi et à la disposition de leurs fleurs; les graines sont nues, trèsgrosses et les barbes fort-longues dans la variété $\mathrm{C}$, tandis qu'elles sont enveloppées et de moitié plus petites dans la variété $A$; mais ce ne sont point là des différences assez grandes pour pouvoir établir des espèces sur de pareils caractères. Quant à la variété $B$. elle n'a jamais été distinguée par personne, malgré que son épi soit assez remarquable par sa rigidité et par la densité de ses épillets.

12. Hord. Distichon.

A. Seminibus vestitis; spica flexili, elongata; spiculis laxe imbricatis.

4. Graines enveloppées; épi flexible, alongé; épillets làchement imbriqués.

Hordeum distichusi J. Bauh. Hist. 2. p. 129.

HORDEUM flosculis lateralibus masculis muticis; seminibus angularibus imbricatis. Lin. Hort. ups. 23.

Hord. distichon Lin. Spec. 1.p. 125. - DeC. Fl. fr. 3. n. ${ }^{0}$ 1682. - Dum. de Cours. Bot. cult. éd. 2. vol. 2. p. 107. n.0 2. - Gaud. Agrost. 1. p. 103. n. ${ }^{0}$ 3. - Host. Gram. Austr. 3. T. 36. Poir. Encyct. 6. p. 613. n. ${ }^{0}$ 3. - Rom. et Schult. Syst. 2. p. T93., n.0 7. A. - Vill. Hist. 2. p. 172. 


\section{1}

(var. à gr. envelop.) - Willd. Spec. 1. p. 473. n.0 3. A. -

Zeocriton distrchon P. Beauv. (d'aprés Rom. et Schult. Syst.)

Fig. Host. Gram. Aust. 3. T. 36.

Exs. Ser. Herb. cer. 12. A. (mat. et comp.)

Obs. Cette variété est très-fréquemment cultivée en Orge d'été, quoique beaucoup moins productive que les orges à six rangs.

Franc. Orge à deux rangs. O. distique. Pamelle, Paoumoule, Baillard.

Allem. Zweizeilige Gerste.

12. HoRDEUM DISTICHON.

B. Seminibus vestitis; spica rigida, brevi; spiculis dense imbricatis.

B. Graines enveloppées; épi roide, court; épillets densément imbriqués.

HorDeUM Hall. Hist. n. ${ }^{0} 1535$.

HoRDEUM astivum spicis explanatis; flosculorum duobus ordinibus fertilibus, intermediis quaternis sterilibus. Hall. Nov. Com. 6. p. 6. n. 20. a. T. 3. f. 24. 25. (C'est aumoins la figure qui se rapproche le plus de ma plante.)

Fig. Hall. Nov, Com. 6. T. 3. f. 24. 25.

Exs. Ser. Herb. cer. 12. B. (mat. et comp.)

Franc. Orge distique, var. à épillets rapprochés. 
12. HoRD. DISTICtion.

C. Seminibus nudis, inflatis; spica flexili; spiculis laxe -imbricatis.

C. Graines nues, enflées; épi flexible; épillets lâchement imbriqués.

Hordeum distichon nudum Lin. Spec. 1.p. 125. n.0 3. - DcC. Fl. fr. 3. n. ${ }^{0}$ 1682. var. B. - Poir. Encycl. 6. p. 603. n. ${ }^{0}$ 3. var. - Raem. et Schult. Syst. 2. p. 793. n. ${ }^{0}$ 7. B. - Vill. Hist. 2. p. 173. (observ.) - Willd. Spec. 1.p. 473.n. ${ }^{0} 3$. B. Enum. 2. p. 1038. n. 0 . B.

Hordeum (AESTIVUm) et var. B. deciduo semine. Hall. Nov. Com. 6. n. ${ }^{0}$ 20, p. 7. Beschreib. des Getreid. n. ${ }^{0}$ 20. p. 66.

Zeocriton distrchum Pal. Beauv. (d'apres Ram. et Schult. Syst: 2.p. 793. n. ${ }^{0}$ 7.)

FrǴ. Arduin Sagg. d. Acad. d. Pad. 3. p. 1. T. 2. f. 4. (d'après Rœm. et Sohult.)

Exs. Ser. Herb. cer. 12. C. (mat. et comp.)

Obs. Cette fort-belle variété mérite bien d'être cultivée. Ses épis sont longs et garnis de trèsgros grains. Elle est préférable à toutes les autres variétés à épis distiques.

Frang. Orge à deux rangs nue. 0 . à café. 0 . du Pérou. O. d'Espagne.

Allem. Nakte Gerste. Polnische zweyzeilige Sommergerste. 
12. HORD. DISTICHON.

D. Spica mutica.

D. Epi sans barbes.

Hordeum disticium ymberbe Roem. et Schult. Syst. 2. p. 793. n. ${ }^{0}$ 7. C.

Je n'ai jamais rencontré la variété à épi dépourvu de barbes, dont parlent MM. Rœm. et Schult.

Franc. Orge distique sans barbes.

\section{HORDEUM ZEOCRITON.}

Spica compressa, pyramidali; aristis valde divergentibus.

Epi comprimé, pyramidal; barbes très - divergentes.

HoRdeum dictum germanis oriza. J. Bauh. Hist. 2. p. 429. (bonne fig. en bois.)

HoRDEUM distichum spica breviore et latiore, granis confertis. Rai. Hist. 2. p. 1243, $n{ }^{0} 2$.

HORDEUM flosculis lateralibus masculis muticis; seminibus angularibus patentibus corticatis. Lin. Hort. ups. 23. n. ${ }^{0} 5$.

Hordeum zeocriton Lin. Spec. 1. p.'125. DeC. Fl. fr. 3. n. ${ }^{0}$ 1683. - Dum. de Cours. Bot. cult. éd. 2. vol. 2. p. 107. n. ${ }^{0}$ 4. - Host. Gram. Aust. 3. T. 37. (tr. bon. fig.) - Pers. Syn. 1. 
p. 108. n. ${ }^{0}$ 4. - Poir. Encycl. 6. p. 603. n. ${ }^{0}$ 4. Rom. et Schult. Syt. 2. p. 793. n.0 8. - Schreb. Gräs. 1. p. 125. T. 17. (fig. noire, trés-belle) Willd. Spec. 1. p. 473. n. ${ }^{0}$ 4. Enum. 2. p. 1038. n. ${ }^{0} 5$.

HordeUM astivum spicis explanatis, flosculorum duobus ordinibus fertilibus; intermediis quaternis sterilibus. Hall. Nov.Com. 6. n. ${ }^{0}$ 20.var. 3. p. 8. T. 3. f. 26 .

Zeocriton commune Palis. Beauv. (d'apres Ræm. et Schult. Syst.)

Fig. J. Bauh. Hist. 2. p. 429. (bonne fig.)

Host. Gram. Aust. 3. T. 37. (tr. bon. fig.)

Schreb. Gräs. T. 17. (fig. noire, tr. bel.)

Hall. Nov. Com. 6. T. 3. f. 26. (belle fig.)

Exs. Ser. Herb. cer. n. ${ }^{0}$ 12. (mat. et comp.)

Obs. Cette espèce n'est pas plus productive que les autres Orges à épis distiques ; et on la rencontre beaucoup moins fréquemment. en Suisse que les autres Orges. - Elle est très-remarquable par son large épi pyramidal comprimć, et par ses belles barbes étendues en éventail.

Franc. Orge en éventail. 0 . pyramidal. 0 . de Russie. O. faux-riz. Riz rustique. Riz d'Allemagne.

Allem. Bart Gerste. 
Angl. Sprat-barbey. Battle-door-barbey. Fulhambarbey. Palney-barbey.

Ital. Orzo di Germanid.

Sued. Skyffcl-korn. Pulmage-korn.

\section{A $\quad \mathrm{V} \quad \mathrm{E} \quad \mathrm{N}$ A.}

Floribus paniculatis; spiculis 2. rarius 3-5. floris; pedicellis apice incrassatis; gluma membranacea, bivalvi; valvulis floribus majoribus, concavis, nervosis, acutis; nervis parallelis; glumella bivalvi; valvulis nervosis; exteriori dorso aristata; seminibus ellipsoüdeis, sulcatis, pilosis.

\section{$\begin{array}{lllllll}A & V & O & I & N & E\end{array}$}

Fleurs paniculées; épillets 2., rarement 3-5. flores; pédicelles renflés au sommet; glume membraneuse, bivalve; valves plus grandes que les fleurs, concaves, nerveuses, aigues; nervures parallèles; glumelle bivalve; valves nervenses, l'extérieure munie vers le milieu du dos d'une longue arête; graines ellipsoïdes sillonnées, poilues. 


\section{T A B L E A U}

DES ESPÈCES ET DES VARIÉTÉS. 3

\section{Avena sativa.}

Panicula æquali; giuma nervosa; glumella glabra; valvula exteriori e medio dorso aristata.

A. Glumella alba, aristata.

B. Glumella alba, mutica.

C. Glumella nigra, aristata.

D. Glumella nigra, mutica.

\section{Avena orientalis.}

Panicula contracta, secunda; gluma nervosa; nervis anastomosantibus; glumella glabra; valvula exteriori e medio dorso aristata.

\section{Avena fatua.}

Panicula laxa, pauciflora; gluma nervosa; glumella barbata; valvula exteriori infra medium longissime aristata.

Ce genre très-naturel se reconnait facilement à ses fleurs disposées, non en épi comme toutes les céréales que j’ai décrites jusqu'ici, mais en panicule. Les glumes dans le peu d'espèces céréales cultivées en Suisse, sont grandes, membrancuses et relevées de nervures nombreuses. Les épillets sont biflores; la fleur la plus inférieure est la plus complette, la plus volumineuse et dans l'état parfait 
munie d'une longue arête, presque toujours genouillée, partant environ du milieu du dos de la valve extérieure de la glumelle, qui embrasse étroitement la graine, laquelle est de forme ellipsoïde et munie de poils soyeux, couchés.

Les arêtes avortent aussi facilement dans les avoines que dans les blés; la même panicule a des fleurs qui en sont munies, d'autres qui en sont pri. vées; le plus souvent dans les deux espèces, cultivées en Suisse comme céréales, des deux fleurs de l'épillet une seule en est pourvue.

\section{AVENA SATIVA.}

Panicula aquali; gluma nervosa; glumella glabra; valvula exteriori e medio dorso aristuta.

Panicule régulière; glume nerveuse; gluınelle glabre; arète naissant du milieu du dos de la valve extérieure.

14. Avena sativa.

A. Glumella alba, aristata.

A. Glumelle blanche, aristée.

Avena ALBa J. Bauh. Hist. 2.p. 432. (mauv. fig.)

Avena vulgaris seu alba. C. Bauh. Theat. p. 469. (d'aprés Hall.)

Avena sativa Lin. (Paniculata, calycilus dis. 
permis, seminibus laevibus), (") Spec 1.p.118. n. ${ }^{0}$ 3. var. B. - DeC. Fl. fr. 3. n. ${ }^{0}$ 1545. B.p. 34. - Dum. de Cours. Bot. cult. éd. 2. vol. 2. p. 122. n. ${ }^{0}$ 5. - Gaud. Agrost. 1. p. 312. - Host. Gram. Aust. 2. T. 59. - Pers. Syn. 1. p. 100. n.0 7. Rocm. et Schult. Syst. 2. p. 668. n.0 4. A. - Sut. Fl. helv. 1. p. 67. n. ${ }^{0}$ 5. - Vill. Hist. 2. p. 14个. n.0 9. - Willd. Spec. 1.p. 446. n. ${ }^{0}$ 13. B. Enum. 1. p. 123. n. $^{0}$ 5. A. -

Avena paniculata; locustis pendulis; floribus cartilagineis, incequalibus, majori aristata. Hall. Hist. $n .^{0} 1494$.

Avena panicula undiquaque sparsa; calycibus flore majoribus; gluma majori carlilaginea. Hall. Nov. Com. 6. p. 16. n. ${ }^{0}$. 25. var. 1. (alba.)

FIG. J. Bauh. Hist. 2. p. 432. (mauv. fig.) Hall. Nov. Com. 6. T. 4. f. 29. (fleur.) Host. Gram. Aust. 2. T. 59.

Exs. Ser. Herb. cer. 14. A. (mat.)

Obs. 1. L'avoine ordinaire ne varie pas moins que les autres céréales, quant à la grandeur. de son chaume ct de sa panicule. Des deux fleurs de chaque épillet, l'une est ordinairement munie d'une arête, ra-

$\left.{ }^{*}\right)$ En disant seminibus laevibus, Linné a voulu parler de la glumelle, qui enveloppe la graine, et non de Ja graine débarrassée de son enveloppe; et c'cst oppositivement avec la glumelle de l' $A$. fatua, qui est très-poilue. 
remont toutes deux; l'autre mutique et de moitié plus petite, n'est presque jamais stérile, mais la graine est de moitié plus petite. Il n'est point rare que quelques épillets d'une panicule soient pourvus de barbes et que d'autres en soient entièrement privées.

Obs. 2. Cette variété et la suivante sont plus fiéquemment cultivées en Suisse que la noire, surtout dans le canton de Berne où l'on rencontre rarement cette dernière. Sa graine est ordinairement emp̣loyée à la nourriture des chevaux, et après avoir subi diverses préparations, à celle de l'homme; (voyez $4^{\circ}$ partie de ce mémoire.) On sème l'avoine très-tard, et c'est la dernière céréale qui se récolte. Franc. Avoine ordinaire, blanche et barbue.

Allem. gemeiner Hafer.

14. Avena sativa.

B. Glumella alba, mutica.

B. Glumelle blanche, sans barbes.

A. satrva B. flosculis omnibus muticis. Roem. et Schult. Syst. 2. p. 668, $n_{0}{ }^{0} 4$.

Exs. Ser. Her. cer. 14. B. (mat.)

Obs. Très-fréquente dans le canton de Berne, mêlée avec la précédente variélé; mais rarement toutes les fleurs sont sans barbes.

Franc. Avoine ordinaire, blanche et sans barbes. 
14. Ávena sativa.

C. Glumella nigra, aristata.

C. Glumelle poire, aristée.

AVENA NIGRA $J$. Bauh. Hist. 2.p.432. (mauv.fig.)

A. Sativa nigra Lin. Spec. $1 . p .118, n .0^{0} 3 . A$. - DeC. Fl. fr. 3. n. ${ }^{0}$ 1545. p. 34. var. A. - Rom. et Schult. Syst. 2. p. 668. n. ${ }^{0}$ 4. var. c. - Vill. Hist. 2. p. 147. - Willd. Spec. 1. p. 446. n.0 13.a.

Avens panicula undiquaque sparsa, calycibus flore majoribus, gluma majori cartilaginea. Hall. Nov. Com. 6. p. 17. n. ${ }^{0}$ 25. II. Tab. 4. fg. 31. a. b. FIG. Hall. Nov. Com. 6. T. 4. f. 31. a. b.

Obs. Très-raremént cultivée en Suisse, ainsi que la variété suivante.

Franc. Avoine ordinaire, noire et barbue.

14. Avena sativa.

D. Glumella nigra, mutica.

D. Glumelle noire, sans barbes.

Exs. Ser. Herb. cer. 14. D. (mat.)

Franc. Avoine ordinaire noire et sans barbes.

\section{AVENA ORIENTALIS.}

Panicula contracta, secunda; gluma nervosa; nervis anastomosantibus; glumella glabra; valvula exteriori e medio dorso aristata.

Panicule 
Panicule contractée ct unilatérale; nervures anastomosées; glumelle glabre: arète naissant du milicu du dos de la valve extérieure.

Obs. Cette espèce ne se distingue de l'Asena sativa que par sa panicule contractée et unilatírale par ses glumes à peine plus grandes, ct dont les nervures s'anastomosent quelquefois; dernier caractère que je n'ai jamais remarqué dans l'diena sativa. Ses fleurs sont souvent dépourtues de barbes, mais je n'en ai jamais rencontré à slumelle noire. Elle est cultivce soit scule, soit mèlée avec l'Avena sativa, de laquelle il est impossible de distinguer les graines; ce qui me fait un peu douter de la solidité de cette espèce.

Avena orientalis Schreb. Spic. Fl. Lips. 52. DeC. Fl. fr. 5. n. ${ }^{0} 15.16$. a. p. 258. - Gaud. Agrost. 1. p. 312. n.0 3. - Host. Gram. Aust. 3. T. 44. - Pers. Syn. 1.p. 100. n.0 6. - Ram. et Schult. Syst. 2. p. 669. n." 5. - Schrad. Fl. Germ. 1.p. 370. n.0 4. - Willd. Spec. 1. p. 446. n.0 12. Enum. 1. p. 122. $\pi .04$.

Avens, panicula heteromalla, calyce flore majori, locustis bifloris, gluma majori cartilaginea. Hall. Nov. Com. 6. p. 18. n." 20. T. 4. f. 32. 33. (A. heteromalla.)

Aven. racemosa Thuill. Fl. par. éd. 2, p. 59.

Fig. Hall. Nov. Com. 6, T. 4. f. 33.34. Host. Gram. 3, T. 44. 
Exs. Ser. Herb. cer. 15.

Franç. Avoine d'Orient. Avoine unilatérale.

Allem. Türkischer Hafer.

\section{AVENA FATUA.}

Particula laxa, pauciflora; gluma nervosa; glumella darbata; valvula exteriori infra medium longissime aristata.

Panicule lâche et pauciflore; glume nerveuse; glumelle hérissée de longs poils nombreux; arête très-longue, naissant au-dessous du milieu de la valvè exterire:

Festuca utriculis lanugine favescentibus. C. Bauh. Pin. 10. Theat. 149.

Atgitops quibusdam aristis recurvis, sive Avena pilosa. J. Barh. Kist. 2. p. 433. (medioc.) Rai. Hist. 2. p. 1254. A. 4.

Avena syluestris pilosa, aristis recurvis. Moris. Hist. 3.p. 209. S. 3. T.7.f.5. (d'iprès les uuteurs.)

Gramen avenaceun, atriculis lanugine flavescentibus. Scheuch. Agrost.p. 239. (Ed. 1775.) T. 5. f. 1. (bonne.)

Avena scininibus basi hirsulis. Lin. Fl. lapp.

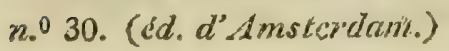


Avena fatua, panicula patente, calycibus trifloris, flosculis basi pilosis. Lin. Fl. Suec. (éd. Slockholm), n. ${ }^{0}$ 101. Spec. 1.p. 118. DeC. Fl. fr. 3. n. ${ }^{0}$ 1547. var. a. - Dum. de Cours. Bot. cult. éd. 2. vol. 2. p. 122. n.0 7. - Gouan Fl. Mionsp. p. 125. n. ${ }^{0}$ 2. - Gaud. Agrost. 1. p. 310. n. ${ }^{0}$ 1. - Host. Gram. Aust. 2. T. 58. - Leers. Fl. Herb. p. 42. n. 90. T. 9. f. 4. (bonne.) - Loisel. Fl. gall. 1. p. 63. n. ${ }^{0}$ 7. - Pers. Syn. 1. p. 100. n. ${ }^{0}$ 10. - Rom. et Schult. Syst. 2. p. 669.n.07. - Schrad. Fl. Germ. 1. p. 373. n. ${ }^{0}$ 6. - Schreb. Beschreib. p. 109. T. 15. (bonne.) Smith Fl. brit. 1. p. 139. n. ${ }^{0} 1$. - Sut. Fl. helv. 1. p. 67. n. ${ }^{0}$ 6. - Wahl. De Veg. n. ${ }^{0} 120$. - Vill. Hist. 2. p. 147. n.0 10. - Willd. Spec. 1. p. 44T. n. 16. Enum. 1.p. 123. n.0 7. -

Avena triantha, locustis patueis, folliculis villosis. Hall. Hist. $n .{ }^{0} 1495$.

FrG. J. Bauh. Hist. 2. p. 433. (méd.)

Moris. Hist. 3. p. 209. S. 8. T. 7. f. 5.

Scheuchz. Agrost. T. 5. f. 1. (bonne.)

Host. Gram. 2. T. 58.

Leers. Fl. Herb. T. 9. f. 4. (bonne.)

Schreb. Beschr. T. 15. (bome.)

Exs. Ehrh. Gra!n. n. ${ }^{0} 28$.

Schl. Cat. 1815, p. 9.

Thom. Cat.

Ser. Herb. cer. 16. A. (compres.)

Obs. Cette espèce n'est d'aucune utilité comme céréale, mais parait se trouver de temps à autre $11^{*}$ 
dans les moissons de la Suisse. Je prie les persomnes qui l'auraient dans leur voisinage, de m'en envoyer des graines mures. - Elle est très-distincte des deux autres avoines par les valves de la glume un peu plus grandes, et surlout par la glumelle garnie dans presque toute la face externe de longs poils roussâtres; une fort longue arête genouillée vers le milieu de sa longueur part du ticrs inférieur de la valve exierne de la glumelle. Je n'en ai pas vu les graines mures, dépourvues de leur glumelle, mais au rapport de $\mathrm{Hajer}$, Gaudin ete. elles paraissent couvertes de poils soyeux, comme les deux espèces précédentes. - L'Avena sterilis Lin. pourrait bien n'être qu'une variété de l' $A$. fatua. Je désirerais avoir des graincs de l'une et d̦e l'autre pour pouvoir les cultiver. - Les exempl. qui sont dans mon Herbarium cereale, viennent de France. Je ne l'ai jamais trouvée en Suisse.

Franc. Avoine follette. Folle avoine. Averon. Avron. Civada couguoüda.

Allem. Flughiaber. Wilder Hafer.

Angl. Beardel Oat-grass. Bearded wild Oats.

\section{P H A L A R I S.}

Floribus spicutis; spiculis unifloris; gluma bivalai; valvulis cymbiformibus; carina alala; glumella quadrivalvi; valvulis internis crustaceis, majoribus, pilosis, semina involventilus. 
Flcurs en épi, épillets uniflores; glume bivalve; valves naviculaires; carìne ailée; glumelle à quatre valves; valves internes crustacées, grandes, poilues, enveloppant les graines.

\section{Phalaris canariensis.}

A. Spica magna.

A. Epi gros.

Piralaris major semine albo. Bauh. Pin. 28. - Moris. Hist. 3. p.186. S. S. T. 3.f. 1. (d'apre's les auteurs.)

\section{Pinlaris J. Bauh. Hist. 2. p. 442.}

Gramen spicatum, semine miliaceo, albo; Tournef. Inst. 1. p. 518.

Phalaris canariensis $L$. Hort. ups. 19. Spec. 1. p. T9. n.0 1. - DeC. Fl. fr. 3. n.0 1490. - Gaud. Agrost. 1. p. 33. n.0 2. - Host. Gram. Aust. 2. T. 38. - Loisel. Fl. gall. 1. p. 37. n.0 1. - Pers. Syn. 1. p. 78. $n .{ }^{0}$ 2. - Rom . et Schult. Syst. 2. p. 402. n. ${ }^{n}$ 1. - Schrad. Fl. Germ. 1. p. 177. n. ${ }^{n} 1$. - Schreb. Beschreib. p. 83. T. 10. f. 2. - Smith Fl. brit. 1. p. 62. n. ${ }^{0}$ 1. - Willd. Spec. 1. p. 326. n. ${ }^{0}$. 1. Enum. 1. p. 83. n.0 1.

FIG. J. Bauh. Hist, 2. p. 442. (mauvaise.) Host. Gram. 2. T. 38. Schreb. Beschreib. T. 10. f. 2. (très-bonne.)

Exs. Scr. Herb. cer. 17. A. (mat. et comp.)

Cette jolie plante, particulièrẹment cultivée pour 
Ia nourriture des oiseaux, est fort remarquable par son épi ovoïde, ses grandes valves naviculaires, à carène áilée, rayées de bandes vertes; sa glumelle à quatre valves, dont les deux externes petites, et Ies deux internes crustacées, velues, grandes, sont appliquées sur la graine, qu'elles enveloppent étroitement; ainsi que par la gaine ventrue de sa feuille supérieure, qui renfermait l'épi. Cette espèce a été surtout bien décrite par MM. Schreber, Schrader, Smith et Gaudin. - Les variétés à graines noires et à graines grises ne sont pas cultivées en Suisse. Franc. Alpiste des canaries à graines blanches.

Allem. Canarien-Gras, Canarien-Saamen.

Angl. Canary-Grass.

Sued. Canarie-Frö.

\section{Phalaris canariensis.}

B. Spica exigua.

B. Epi petit.

J'ai obtenu cette variété en semant la même graine (qui a produit la variété précédente) dans un terrain aride et mal préparé. Tous les caractères de l'espèce s'y retrouvent; toute la plante (les graines et les parties de la fieur excepté, qui ont conservé leur grandeur et leurforme) a un aspect apauvri; l'épi luimême au lieu d'avoir une centaine de fleurs, n'en a souvent que dix à quinze. - Jl est probable que c'est à peu près dans cet état que cette espèce se trouve spontanément.

Franc. Alpiste des canaries apauvri. 


\section{$\begin{array}{lllllll}0 & R & Y & Z & A\end{array}$}

Floribus paricielatis; spieulis unifloris; glume jivalvi; valvis angustis, exiguis, acutis, cymbiformibus; glumella crustacea. cymliformi, bivalvi, ad lentem rugosa-punctata; valvula exteriori majori, quinquangulata; seminibus ovoüdeis, olıtusis, compressis, angulosis.

Fleurs paniculées; épillets miffores; ģlume bivalve; valves étroites, petites, aigues, naviculaires; glumelle crustacée, naviculaire, bivalve, rugueuse. ponctuée (à la loupe); valre extérieure grande, quinquangulaire; graines ovoïles, obtuses, comprimées, anguleuses.

Ce joli genre de l'Hexandrie digynie de Linné, n'est point cultivé en Suisse, mais ayant pu noen procurer un assez grand nombre d'exempl. qui ont été récoltés en Picinont, et que j’ai joints à mon Herharium cereate; j’ai cru devoir y ajouter sa description, sa synonymic et quelques détails sur sa culture.

Les fleurs sont elisposées en panicuie làche; les épillets sont uniflores; la glume est formée de deux valves acérées, étroites, naviculaires, très-petites en proportion des deux valves de la glumelle, qui sont crustacées, naviculaires, poilues et hérissées de petites aspérités ponctiformes; l'extérieure est relevée de cing côtes longitudinales et terminée par une lonzue arête scabre; la valve intérieure de la glumelle est plus petile que l'extérieure, rugueuse, mais non 
striée. La graine est ovoïde-comprimée, obtuse, relevée longitudinalement de six còtes. Les feuilles d'ailleurs ressemblent à celles des autres graminées.

\section{OrYZa SATIVA.}

Oriza $J$. Bauh. Hist. 2. p. 451. (bonne fig.) Tournef. Inst. 1. p. 513. Tab. 296. (bonne fig.)

Oryza sativa Lin. spec. 1.p. 475. - DeC. Syzn. n. 1494. * - Del. Fl. ogypt. n. ${ }^{0}$ 390. - Pers. Syn. D. p.394. - Willd. Spec. 2. p.247. -

D'après Willdenow Spec. 2. p. 247., il existe un grand nombre de variétés du riz; voici la note qu'il donne: "Possideo 18 varietates hujus graininis seminibus ovatis, ellipticis, oblongis, subrotundis, brevibus, sulcatis, albis, nigris, fuscis, muticis, aristatis etc. quce sensim una in alteram transeunt, ut limites nulli observentur." Je prie les personnes qui seraient voisines des rizic̀res, de vouloir bien m'en récolter un grand nombre d'exempl. de la variété la plus commune, ainsi que de tous les états différens qui pourraient s'offrir.

\section{Culture des rizières en Toscane.}

"La rizière est disposée sous la forme d'un grand parallćlograme, divisé lui-mème en un grand nombre d'aires régulières, enfoncées, sćparées par des sentiers élcvés : sur les deux longs côtés du parallélograme, cst un large fossé; l'un ůn peu plus 
élevé que la rizière, et qui sert à y amener l'eau, l'autre un peu plus bas qu'elle, et qui sert de canal de décharge. Au mois de Mars, le terrain étant privé d'cau depuis long-temps, on le laboure avec l'espèce de bèche appelée Venga; on n'y met point d'engrais; on fait entrer l'eau dans les aires de manière à ce qu'il $y$ en ait environ dix-huit centimètres an-dessus du sol, qui devicnt lui-nême tout-à-fait. boueux. On y sème le riz à la volée, et en mème quantité qu'on sèmerait du blé sur la même surface. Le jeune riz reste environ un mois avant de s'élever au-dessus de la surface de l'eau. Au mois de Mai on fait passer dans les rizières des femmes pour arracher les mauvaises herbes qui y sont trèsabondantes. - Le riz n'est sujet ni au charbon, ni à la carie; sa seule maladie est que quelque fois ses glumes (glumelles) deviennent vides et blanchàtres, à peu près comme dans le blé éventé; on le nomme alors Riz annebiato, c'est-à-dire touché par les brouillards, car l'opinion des cultivateurs est que cet état morbifique est dû à l'action des brouillards; je le concevrais sans peine s'il était seulement question des brouillards, qui auraient licu à l'époque de la fécondation, mais les paysans leur attribuent cet effet pendant toute l'annce, et il devient incompréhensible à mes yeux. Le Riz murit au milieu de Septembre; alors on enlève l'eau du champ et on moissomne le riz en coupant la tige à moitié hauteur; la partie inférieure reste sur place, où clle pourrit et sert d'engrais pour l'année suivante. La partie supéricure se met en petites manipules ou javelles; on le porte ainsi à la fattorie, où on le 
secoue pour le débarrasser des grains murs; on le bat ensuite avec des fléaux comme le blé, pour détacher le reste. Dans ces opérations on n'obtient encore que le grain enveloppé de sa bâle (glumelle); pour l'en dépouiller, on le fait d'abord passer sous une meule disposée à peu près comme celle qui sert à moudre l'orge, ce qui sert à enlever la glume (glumelle); puis on le place sous des foulons armés de pointes de fer, ce qui sert à enlever les derniers débris de glumelle, et à blanchir le riz; enfin on le passe au crible, et il devient alors propre à être livré au commerce. Toutes ces opérations font que, quoique le riz rapporte plus que lc blé en quantité, il donne souvent moins de profit réel." (DeCandolle 3." Rapport sur un voyage botanique et agronomique, p. 46.)

\section{Culture des rizidres en Egypte.}

"Les Egyptiens cultivent une grande quantité de riz pour leur consommation et pourl'exportation. Aucun historien ancien n'a parlé du riz d'Egypte, et je suis porté à croire, avec Hasselquist, (Voyage dans le Levant, part. 1.p. 163.) que cette culture ne remonte point chez les Egyptiens au-delà du temps des Califes, qui favorisèrent l'introduction des plantes étrangères. On choisit dans le Delta pour semer le riz le grain le plus beau, on en remplit des sacs faits avec les feuilles de dattiers, (ces sacs sont appelés couffis) on les porte dans un canal ou dans un réservoir près des roues d'arro- 
sement. Ces couffes restent à moitié plongées dans l'eau, et $y$ sont retournées chaque jour. Le riz commence ainsi à germer. On sort les couffes de l'eau le 5 ou $6 .^{\circ}$ jour'; on les vide en mettant le grain par tas sur une couche de trèfle frais, et en couvrant le tas avec le trèfle. On ne remue alors le riz qu'au bout de vingt-q̧uatre heures, on l'étend et on le laisse pendant un jour recouvert die trèfle, que l'on ôte le soir; puis il reste exposé à la rosée de la nuit. On le sème le matin dans un champ qui a été couvert d'eau, et d'où elle ne s'est pas même entièrement écoulée. On met par la suite plusieurs fois le champ à sec à de courts intervalles pour forcer le riz à prendre racine et à ne pas submerger. Plus tard on nétoye le champ de diverses mauvaises herbes, et en même temps on arrache aussi quelques touffes de riz trop épaisses, que l'on replante aux endroits trop clairs, on dans un cluamp voisin, préparé à cet effet. Cette transplantation est facile dans la boue, d'où l'on retire le riz par ses tiges et sur laquelle on le replante. L'eau, dans laquelle baigne le pied du riz, jusqu'à ce que le grain soit mur, provient des machines d'arrosement, qui servent à la puiser dans le Nil. Elle se distribue aussi d'elle-même an temps de l'inondation, et son cours est règlé par les digues, qui protègent les champs."

"La récolte du riz se fait en Octobre, après qu'il est resté sept mois en terre; on le bat sous le Noreg; (Voyez Descript. de l'Egypte, arts et métiers, Table 8 et 9.) le grain séparé de la paille 
conserve sa glumelle, ou enveloppe florale, fermement atlachée, comme celle de l'orge, et on l'appèle dans cet tat Rouz cha'yr (riz en orge). Le riz suffisamment pilé dans des mortiers, est criblé pour le séparer de son enveloppe et des grains ćcrasés. On mèle le riz avec du sel ordinaire sec, afin de l'empêcher de se gàter." (Descript. de l'Egypte. Histoire des plantes cultivées en Egypte par A.R. Delile, méinoire sur les céréales $p .16$.

\section{$\begin{array}{llllllll}\mathrm{P} & \mathrm{A} & \mathrm{N} & \mathrm{I} & \mathrm{C} & \mathrm{U} & \mathrm{M} \text {. }\end{array}$}

Floribus spicatis vel paniculatis; spiculis unifloris; gluma trivalvi; valualis membranaceis, nervosis; glumella bivalvi; valvulis crustaceis semina involventibus.

\section{$M \quad I \quad L \quad L \quad E \quad I$.}

Fleurs en épi ou en panicule; épillets uniflores; glume trivalve; valves membraneuses, nerveuses; glumelle bivalve, valves crustacées, enveloppant la graine.

Les genres Panicum et Setaria de Mr. Palisot de Beauvois, ne me paraissent guìre pouvoir en constituer vraiment deux. Des deux espèces cidessous, l'une est un Panicum et l'autre une Setaria. 
Pal. Beauv. (Genres arloptés par MM. Rœm. et Schult. Syst.) Toutes les deux ont une graine enveloppée par les deux valves crustacćes de la glumelle; toutes les deux ont trois valves membrancuses, nerveuses à la glume; il n'y aurait donc que l'inflorescence, (le genre Setaria a ses fleur's en épi, le genre Ponicum les a disposécs en panicule) avec les espèces de soies roides, qui partent de dessus les pédicelles, dans le genre Setaria et qui manquent au genre Panicum, qui les différencieraient; et je crois que ce ne sont là que des caractères de groupes, mais non de genres.

D'ailleurs le Panicum miliacetem a ses graines ovoüdes et lisses, tandis que le P. itclizm (Séaria italica. Pal. Beauv.) les a presque rondes et chagrinées. N'ayant examiné soigneusement que ces deux espèces, je suis loin de pouvoir décider, mais je crois qu'il faudrait pour qu'on put s'appayer sur celle forme sphéroïle et sur la rugosité du genre Seturia que les graines des Seturia eussent toutes les mêmes caractères, auxquels alor's on pourrait joindre le caractère, bien faible, de l'inflorescence. Le hile dans mes deux espèces est très - grand et très-profond.

Je n'ai pu trouver sur le frais la fleur mâle ou neutre, dont quelques auteurs font mention : pentêtre auront-ils regardéla valve la plus inféricure de la glume (qui est plus ou moins ćcarlée des deux autres, qui elles-mèmes sont alternes) comme une fleur; mais je n'ai jamais pu voir de rudiment d'é- 
tamines. Peut-être qu'au lieur d'établir des genres sur des caractères peu solides, il vaudrait mieux former des groupes bien marqués, qui mèneraient au même but.

\section{T A B L E A U}

DES ESPÈCES ET VARIÉTÉS.

\section{Panicum miliaceum.}

Floribus paniculatis; glumæ valvulis acutis; pedicellis nudis; seminibus ovordeis, laevibus.

A. Panicula nutante; seminibus stramineis.

B. Panicula erecta ; seminibus badiis.

\section{Panicum italicurn.}

Floribus spicatis; ginmæ valvulis obtusis; pedicellis bracteatis; bracteis longis, setaceis ; seminibus globosis, transverse-rugosis.
A. Spica elongata; seminibus stramineis.
B. Spıca sub ovoldea; seminibus stramineis.
C. Spica sub ovoïdea; seminibus aurantiacis.
D. Spica sub ovoüdea; pedunculis violaceis.
E. Spica exigua. 
19. PANICUM MILIACEUM.

Floribus paniculatis; gluma valvulis acutis; pedicellis nudis; seminibus ovoideis, laevilus.

Fleur's paniculées, valves de la glume pointue; pédicelles nus; graines ovoïdes, lisses.

19. Panic. millaceum.

A. Panicula nutante; seminibus stramineis.

A. Panicule penchée; graines d'un jaune pâle.

Mrlium semine luteo. C. Bauh. Pin. 26. - Rai. Hist. 2. p.1251. - Tournef. Inst. p. 514. T. 298. L. (figuré jeune.)

Minium J.Bauh. Hist. 2. p. 446. (Jig.diminuée, et médiocre.)

Panicum milaceum Lin. Spec. 1. p. 85. n. 23. - DeC. Fl. fr. 3. n. ${ }^{0}$ 1502, p. 15. (var. semine luteo.) - Gaud. Agrost. 1.p. 25. n.0 8. - Hoffm. Fl. Germ. (1791.) p. 22. n.0 11. - Host. gram. Aust. 2. p. 16. T. 20. - Loisel. Fl. gall. 1. p. 40. n. ${ }^{0}$ 10. - Ram. et Schult. Syst. 2. p. 434. n. ${ }^{0} 33$. - Schrad. Fl. Germ. 1. p. 245. n. ${ }^{0}$ 6. - Willd. Spec. 1. p. 348. n. 49. Enum. 2.p. 1033. n.0 17.

Panicuan milium Pers. Syn. 1. p. 83. n.0 50.

Frg. J. Bauh. Hist. 2. p. 446.

Tournef. Inst. T. 299. L.

Host. Gram. 2. T. 20.

Exs. Ser. Herb. ccr. 19. A. (compr.) 
Obs. Cette variété est facile à reconnaître à sa panicule penchée, à scs graines couleur paille, et aux gaines de ses feuilles hérissées de longs poils mols et distants. Elle se sème toujours au printemps, et réuısit mème dans les plus mauvais terrains. Les paysans font gruer ses graines et en font des soupes, des bouillies fort-bonnes.

Franc. Mil, Millet, (variété à graines jaunes.)

Allem. Hirsen Fennich, Hirss.

Angl. Myle.

Espag. Milhe.

Bohem. Proso.

19. Panic. miliaceum.

B. Panicula erecta; seminibus badiis.

B. Panicule dressée; graines brunes.

Milium semine nigro. C. Bauh. Pin. 26.-Rai. Hist. 2.p. 1251.n. ${ }^{0}$ 3. - Tournef. Inst. 1.p. 514. -

Milium semine nigro spadiceove. J. Bauh. Hist. 2. $p .446$.

Panicum miliaceum, semine nigrescente. DeC. Fl. fr. n. ${ }^{0}$ 1502. - Loisel. Fl. gall. 1.p. 40. var. B. - Rocm. et Schult. Syst. 2. p. 434. n.0 33. Schrad. Fl. Germ.1.p. 247.

Exs. Ser. Herb. cer. 19. B. (compr.)

Obs. 1. 
Obs. 1. Cette variété est très-reconnaissable à sa panicule làche et dressée, à son chaume plus ferme, et à ses graines d'un brun noirâtre.

Obs. 2. Il parait, d'après les frères Bauhin et Ray, qu'elle ćtait assez fréquemoment cultivée autrefois dans l'Aarguvie; j"ignore si on l'y trouve encore; je ne l'ai vue que bien rarement dans le canton de Berne.

Frane. Millet, (variété à graines brunes.)

\section{PANICUM ITALICUM.}

Floribus spicatis; glumellce valvulis oblusis; pedicellis bracteatis; bracteis longis, setaceis; seminibus globosis, transverse rugosis.

Fleurs en épi; valves de la glumelle obtuses; pédicelles bractées; bractées longues, sétacées; graines globuleuses, transversalement chagrinées.

A. Spica elongata; seminibus stramineis. A. Epi alongé, graines pailles.

Panicum gerananicum, sive panicula minore, flava. C. Bauh. Pin. 27. - Tournef. Inst. 1. p. 515. 3. T. 298. $M$. (bonne figure.)

Pantcum vulgare $J$. Baul. Hist. 2. p. 440. (fg. méd.)

Panicum italicum Lin. Spec. 1. p. 83. n.0. 6. (Panicum, spira composita; spiculis glomeratis, 
setis immixtis, pedunculis hirsutis. - DeC. Fl. fr. 3. n. ${ }^{0}$ 1499. var. B. - Gaud. Agrost. 1. p. 20. n.0 4. -

Fig. J. Bauh. Hist. 2. p. 440.

Tournef. Inst. 3. T. 298. M.

Exs. Ser. Herb. cer. 20. A. (mat.)

Obs. 1. Cette espèce, très-distincte de la précédente, par ses fleurs en épi, par les bractées sétacées, qui accompagnent les pédicelles, les longs poils, qui recouvrent le rachis, et ses graines globuleuses et striées, offre un grand nombre de variétés, dues à la culture. Les bractées, qui accompagnent les pédicelles, varient beaucoup de longueur et dépassent à peine dans les variétés suisses les fleurs, tandis que d'autres fois elles hérissent l'épi. Comme on s'est attaché jusqu'à présent à des caractères peu importants pour distinguer les Panicum italicum et germanicum, Willd. Spec. 1. p. 336. n. ${ }^{0}$ \% et 8., que MM. Rom. et Schult. Syst. 2. p. 492. n. 0 14. et 15., ont adoptés; il me semble fort probable que les dcux espèces de ces auteurs ne sont que la même plante. Je prierais les personnes, qui auraient d'autres variétés que celles qui se trouvent dans mon Herbarium cereale, de vouloir bien me les communiquer. Toutes les miennes ont les valves de la glume de la même forme, ainsi que celle des graines; la valve de l'épi et sa couleur seules varient.

Obs. 2. Cette espèce est cultivéc en Suisse plutôt pour la nourriture des oiseaux que pour celle de 
l'homme, cependant les paysans la vendent souvent toute gruée au marché de Berne. - Cette variété est plus fréquente dans les cantons de Genève et de Vaud, que dans celui de Berne; cependant j'en ai vu cette année dans cette dernière ville un plus grand nombre, qui approchait de la variété cultivée à Genève, que les années précédentes; ce que j'attribue à la température, qui a été plus favorable à son parfait développement.

Franc. Panic ou millet des oiseaux, variété à épi alongé.

Allem. Welscher Fennich.

20. Panicum italicum.

B. Spica sub ovoüdea; seminibus stramineis.

B. Epi presque ovoïde; graines pailles.

Obs. Cette variété n'est remarquable que par la brièveté de son épi; d'ailleurs toutes les parties ont la même conformation, que la variété précédente. C'est l'état que l'on trouve le plus communément au marché de Berne.

Franc. Var. à épi court et presque oroüde du Panic des oiseaut.

20. Panic. italicum.

C. Spica subovoïdea; seminibus aurantiacis.

C. Epi presque ovoïde, graines orangées. 
Obs. Cette variété, assez rarement cultivće, est remarquable dans l'état frais par son épi rougeàtre et par ses graines orangées à leur maturité. Franc. Var. à graines orangées du Panic des oiseaux.

\section{Pantcum italicum.}

D. Spica subovoïlea; pedunculis violaceis.

D. Epi presque ovoïde; pédoncules violàtres.

Obs. Variété à pédoncules pourpres, dans l'état frais, et violàtres, dans l'état de dessication.

Franc. Vaì. à pédoncules violâtres du Panic des oiseaux.

20. Panitc. italicum.

E. Spica exigua.

E. Epi apauvri.

Obs. Variété obtenue par la culture. Elle avait été semée dans un terrain sablonneux très-aride. Franç. Varićté apaurric du Panic des oiseaux.

\section{A Y S.}

Floribus monoïcis; spiculis masculis bifloris paniculam terminalem constituentibus; femineis unifloris spicis lateralibus insidentibus; stylis longissimis; seminibus subrotundis, lnevibus, seriatim dispositis. 


\section{$M A \ddot{I} S$.}

Fleurs monoïques; épillets mâles biflores, formant une panicule terminale; femelles uniflores, disposées en épis latéraux; styles très-longs; graines presque rondes, lisses, sériées.

Obs.1. Ce beau genre, de la Moncecie Triandrie, est remarquable par ses fleurs inâles paniculées, dont la glume est biflore et bivalve; la glumelle membraneuse, transparente, bivalve et mutique, et trois étanines. Les fleurs femelles sont disposées en épi, enveloppées de larges bractées au nombre de quatre à six; ces bractées sont foliacées pendant la fleuraison, puis sèches et scarieuses, et forment viaiment la glume. Les fleurs, disposées par séries verticales, sont formées de quelques valves de la glumelle membraneuse, courte, quelquefois ciliée, et d'un gros ovaire, terminé au sommet par un long style, qui dépasse la glume, et qui se transforme en un gros grain, quadrilatère à la base et arondi au sommet.

Obs. 2. Le nom de Zea ayant très-anciennement été donné au Triticum Speltu, et peut-être au T. amyleum, j'ai adopté à l'exemple des Bauhin, Tournefort et DeCandolle le nom de May's, qui ne fut connu que postérieurement, et qui était cultivé par les Américains lorsqu'on fit la découverte du Nouveau-Monde. 
21. Mays vulgaris.

A. Spica simplici; granis aureis.

A. Epi simple; graines dorées.

Frumentum indicum Mays dictum. C. Bauh. Pin. 25.

Triticum peruvianum $J$. Bauh. Hist. 2. p. 454. (fig. Médioc.)

MAYS Tournef. Inst. 1.p. 531. 2. T. 303. 304. 305. (bonnes fig.)

ZeA Mays Lin. Spec. 2. p. 13TS. - Delit. Fl. regypt. n.0 881. (Descript. de l'Egypt.) - Gouan. Fl. Monspel.p. 111. - Loisel. Fl. gall. 2. p. 626. Pers. Syn. 2. p. 533. - Vill. Hist. 2. p. $180-$ Willd. Spec. 4. p. 200. Enum. 2. p. 952. -

MaYs zea DeC. Fl. fr. 3. n. ${ }^{0}$ 1694. p. 9 S.

FIG. J. Bauh. Hist. 2. p. 454.

Tournef. Inst. 2. T. 303. 304. 305.

J. Gessn. Tab. phyt. T. 5. f. 925. (belle.)

Exs. Ser. Herb. cer. 21. A. (graine.)

Obs. 1. Cette variété est la plus cultivée de toutes, particulièrement en Valais, et dans quelques parties du canton de Vaud. Sa farine est jaune et le son formé de grandes plaques du péricarpe et du périsperme, qui servent en Italie à emballer les objets fragiles. La farine est employée à préparer ce qu'on appèle Polenta, Poulinte, espèce de bouillie épaisse, qui sert en partie à la nourri- 
ture des pauvres en Piémont et en Italie; on en fait aussi des soupes, des bouillies, des galettes, et mème du pain. C'est une nourriture très-substantielle. Les Egyptiens récoltent souvent les épis de Ma ïs à demi murs pour les manger rotis.

Obs. 2. Les expositions chaudes et les terrains gras conviennent bien à cette plante, qui réussit assez mal dans les parties humides de la Suisse, ce qui a en partie empêché sa culture de se répandre. Après l'entière fleuraison de la panicule des fleurs mâles, on la coupe au-dessus de l'épi supérieur fumelle, et on la donne aux bestiaux. Les grandes valves coriaces de la glume servent dans le Midi à faire des paillasses. On cultive souvent aussi le Maïs après la récolte des blés pour le donner comme fourage aux boufs et aux vaches, qui en sont très-friands.

Franc. Maïs ordinaire, variété à grains dorés.

Allem. Gemeiner Mays.

Arab. Tourky, dourah châmy, dourah kyrân.

21. Mays vulgaris.

B. Spica simplici; granis purpureis

B. Epi simple; graines pourpres.

MaYs granis rubris. Tournef: Inst. 1.p.531. -

MAYS ZEA granis purpureis. DeC. Fl. fr. n.01694. Exs. Ser. Herb. cer. 21. B. (graines.)

Cette variété est remarquable par ses graines 
d'un beau pourpre foncé; elle est plus rarement cultivée que la précédente, et n'est qu'accidentelle.

Franc. Maïs ordinaire, variété à grains pourpres.

21. Mays vulgaris.

C. Spica simplici; granis variegatis.

C. Epi simple; grains panachés.

Mays zea granis variegatis. DeC. Fl. fr. $n 0^{0}$ 1694.

Exs. Ser. Herb. cer. 21. C. (graine.)

Obs. Cette jolie variété semble être une hybride des deux variétés précédentes. Le même épi offre quelque fois des grains pourpres, d'autres entièrement jaunes, et le plus grand nombre est panaché de lignes pourpres sur un fond jaune.

Franç. Maïs ordinaire, variété à grains panachés.

21. MaYs vulgaris.

D. Spica ramosa; granis aureis.

D. Epi rameux; grains dorés.

Frumentum indicum, spica divisa, seut polystachites. Boccone Icones et descriptiones, p. 33.f.1.

MAYs spica multiplici. Tournef. Inst. 1. p. 531.

MAYS ze spica fominea ramosa. DeC. Fl. fr. n. ${ }^{0} 1694$.

Frg. Bocc. Icon. p. 32. f. 1 . 
Obs. J'ai rencontré deux fois cet état en Valais; l'épi, aulicu d'être simple, est rameux, et plus ou moins déformé; monstruosité qui se remarque aussi dans d'autres genres de la inême famille. (Triticum turgidum, Tr. amyleum, Secale cereale, Lolium perenne.) Mr. de Bernaud, Bibliot. physicoéconomique, année 181s, Juin, p. 401, en cite de nouveaux exemples : "Un picd de cc Maïs qui s'est trouvé semé en iS17 dans des platras et à peu près sous une goutlière, chez Mr. le comte Francois de Neuchateau, a eu une végétation luxuriante, et a produit des épis rameux, absolument semblables à la figurc citcé. Cet accident n'est pas rare dans le Piémont, où la culture du Maïs est très-étendue. Au rapport d'un grand proprićtaire de ce pays, le Maïs, abondamment fumé et placé dans des circonstances favorables, domne quelque fois ces produits extraordinaires. Il reste maintenant à savoir, si cette bizarrerie se soutiendra dans les plantes qui proviendront des grains de ce Maïs à épis rameux. C'est ce que Mr. Francois de Neuchatean se propose de vérifier." Il serait aussi fort-possible, que dans le Maïs l'extrémité de l'épi cut été blessée dans sa jeunesse, ou que quelques obstacles l'eussent forcé de se diviser.

Franc. Maïs ordinaire à épi rameux.

21. Mays vulgaris, (Quarantino.)

E. Caule humili; spica exili.

E. Tige basse, épi pelit.

Exs. Ser. Herb. cer. 21. E. (compr.) 
Obs. 1. Cette variété, appelée Quarantin, par quelques cultivateurs, a une végétation très-prompte; et toute la plante reste très-petite. Elle est semée, par quelques agronomes après la récolte de l'orge d'hiver, et murit encore avant les froids, ou est donnée en verd aux bestiaux.

Obs. 2. Tournef. et Mr. DeCandolle indiquent plusieurs autres variétés que je n'ai jamais rencontrées en Suisse. Je prie instamment les cultivateurs, qui auraient celles que j'ai indiquées, ou d'autres, de m'en envoyer des épis murs.

Franc. Maïs quarantin.

Quelques grandes plantes, qui se trouvent ordinairement dans les moissons, se sont tellement propagées dans quelques terrains, qu'elles occupent autant de place que les céréales elles-mêmes, et les gênent beaucoup dans leur développement; ce sont - 22; la Centaurea Cyanus L. (Bluet, Bleuet, Barbot) 23 ; le Papaver Rhaeas L. (Coquelicot, Ponceau) - 24; le Lychnis githago DeC., ou Agrostema githago L. (Nielle) $\left(^{*}\right)$ et 25 , le Cynosurus echinatus L. (Cynosure hérissée.) Ser. Herb. cer. n. ${ }^{0}$ 26.) Malgré que leurs graines réduites en farine ne produisent aucun effet nuisible sur l'homme; elles donnont au pain une teinte brune et étouffent les blćs.

(*) Je donnerai ces plantes desséchées dans la seconde livraison de mon Herbarjum cereale, pour lequel je ramasse des matériaux. 
Plus la culture est soignée dans une contrée, plus ces plantes disparaissent. Il serait peut-être très-difficile d'en trouver une seule dans le terrain de l'Institut d'agriculture de Mr. Fellenberg. Il offre la preuve de ce que peut un cultivateur zèlé qui, par le criblage et le sarclage est parvenu à les faire disparaitre de ses belles moissons.

Une plante non moins fréquente dans les moissons, mais qui n'est pas aussi innocente qu'elles, est le Lolium temulentum L. - Ser. Herb. cer. 27. (l'ivraie, betäubender Lolch), dont les graines produisent des accidents que l'on dit graves. Je désirerais bien que les cultivateurs, qui l'ont dans leur terrain, m'en envoyassent quelques livres pour faire des expériences sur les animaux. Je ne l'ai trouvée que fort-rarement dans le canton de Berne parmi des orges.

\section{MALADIES DES CÉRÉALES.}

\section{PARTIE.}

Il parait actuellement prouvé que sept champignons parasites, dont cinq surtout sont très-nuisibles aux graminées, produisent les maladies des céréales. Long-temps on a accusé le sol, les engrais, les vicissitudes atmosphériques d'être la cause de ces maladies; elles me semblent très-clairement expliquées dans l'Extrait d'un mémoire sur les champignons parasites, du Professeur DeCandolle, lu à 
I'Institut le 26 Octobre 1806 , et inséré dans les Annales du muséum d'histoire naturelle 9. p. 5674. (1807.) Ce savant profond y donne une théorie simple de ces maladies. Les cinq champignons vraiment dévastateurs de nos moissons sont:

Uredo carbo (Charbon, Nielle, Brand.)

Uredo maYadis (Urédo du Maïs.)

Uredo caries (Carie, Kornfrass, Kornfäule.)

Uredo rubigo-vera (Rouille, Rost.)

Sclerotium clavus (Ergot, Mutterkorn.)

Les autres, qui n'influent que faiblement sur la maturation des céréales sont:

Puccinia graminis.

Sphaeria punctiformis Graminaria.

Ces maladies des céréales sont produites, non par des vices d'or'ganes, mais par l'épuisement que produisent des champignons parasites sur la totalité ou sur une parlie de ces végétaux.

Une plante ne peut être propagée : 1.0 que par ses graines, qui, rencontrant les circonstances convenables pour leur développement, germent et produisent des êtres semblables à eux-mèmes, ou 2. ${ }^{\circ}$ que par des portions d'une plante séparées de l'individu et qui, munies de racines ou sans racines d'abord, se développent et produisent un individu scmblable à cclui d'où elle a été détachéc. Ce second mode de reproduction ne peut être at- 
tribué aux champignons, surtout à ceux qui sont vraiment parasites, ou qui croissent sur d'autres individus encore doués de la vic; le premier est donc le seul admissible, malgré qu'on n'ait pas encore de preuves certaines sur leur propagation. Deux théories ont été données sur cette propagation des champignons parasites; l'ume par Sir Joseph Banks, l'un des savans naturalistes de l'Angleterre; l'autre par Mr. DeCandolle.

Sir Banks pense que les graines, d'une ténuité prodigieuse, entrent dans les feuilles par les pores corticaux; Mr. DeCandolle, qu'elles sont introduites par les racines et disséminées dans la plante par la sève. $\left(^{*}\right)$

"Les pores corticaux sont, comme on sait, épars sur toute la surface herbacée des plantes; ils servent généralement à la transpiration, et, dans quelques circonstances, à l'imbibition des vapeurs et des gaz. Ainsi les graines des champignons, qui flottent dans l'air, pourraient bien entrer dans ces pores et se développer sous l'épiderme. Ce soupçon semble même d'autant plus plausible, que généralement les champignons parasiles naissent à la surface inférieure des feuilles, qui est aussi celle où l'on trouve le plus de pores corticaux, et que quelque fois les champignons sortent de ces pores, comme Mr. Banks l'a remarqué relativement à la Puccinia

$\left(^{*}\right)$ Les morceaux accompagnés de guillemets sont de Mr. DeCandolle, tout le reste n'est qu'une suite de sa théorie. 
graminis. Mais cette théorie est sujetle à des objections, qui me paraissent importantes."

"1.0 Il existe plusieurs feuilles, qui ont des pores sur les deux surfaces, et qui n'émettent de champignons que sur l'une d'elles: Telles sont la Puccinia graminis, la Puccinia dianthi, ct l'Uredo candida. - 2.0 Il y a quelques plantes qui n'ont de pores qu'à la surface inférieure des feuilles, et qui ont les champignons à la surface supéricure; tels sont les Puccinia ribis, et la plupart des espèces du genre Xyloma. - 3.0 Les champignons parasites naissent souvent sur des organes dépourvus de pores corlicaux. Ainsi on trouve l'Uredo rosae sur la base de l'ovaire et sur le pédicelle; l'Uredo carbo sur les ovaires; la Puccinia adoxae, P. ficariae etc. sur le pétiole, les nervures, et quelquefois sur les rameaux ligneux des plantes qu'elles attaquent 4. ${ }^{\circ}$ Dans quelques plantes, telles que le Rubus idaeus et le Tussilago farfara, les champignons parasites naissent sous l'épiderme, lequel est recouvert par un duvet serré, comme feutré, et qui repousse l'eau. $-5 .{ }^{0}$ Quelques parasites naissent sur des plantes dépourvues de pores corticaux : tel est l'Uredo $m y$ cophila et l'Aecidium peltigerae. - 6.0 On sait que les injections colorées passent bien plus facilement par les racines, quoique leurs pores soient encorc mal connus, que par les pores corticaux, qui sont cependant bien visibles."

"D'après ces observations, il est plus plausible de penser que les graines des champignons parasites 


\section{1}

tombent à terre à leur maturité, se mêlent avec le terreau, sont entrainécs par la sève aspirée, entrent dans les racines, montent le long ảu corps ligncux, arrivent avec la sève dans les parties herbacées; que là, trouvant une position, ou une nourriture convenable, ces germes se développent. On voit d'abord la couleur de la feuille s'altérer un peu, puis l'épiderme sc soulìve et se fend. Si les parasites sont plus communs à la surface, qui porte les pores, c'est que la sève, qui se dirige ver's eux, y conduit natureliement les graines. Si on en trouve ailleur's, c'est que la sève parcourt successivement tout le végétal."

"On ne doit point s'effrayer ici de l'extrême ténuité que je suppose dans les graines de nos champignons. En effet, une plante entière de Puccinia n'a pas un douzième de millimètre de longueur, chaque loge n'a pas un centième de millimètre, et cette loge renferme aumoins cent petits globules, à peine visibles au microscope, et surement plus petits que certaines molécules terreuses ou colorantes que nous royons s'introduire dans les vaisseaux des plantes."

"Au moyen de cette théorie, on explique faciIement plusieurs faits, dont la précédente ne peut rendre raison. - 1.0 C'est $u$ fait, qui me parait constant, que si dans un certain terrain les plantes sont attaquées d'un parasite, elles le sont encore les années suivanies. Or on rend bien plus facilement raison de ce fait, en admettant que les grai- 
nes sont mêlées avec le terreau; quicn les supposant voltigeant dans l'atmosphère. J'ai vu pendant plusieurs années deux jardins, séparés seulement par un espace de quelques toises, dont l'un avait tous ses poirier's infestés de l'Aecidium cancellatum, et l'autre avait tous ses poiriers sains. L'Erythronium dens-ranis, qui croit dans un petit bois près de Genève, $y$ a été observé par Mr. Vaucher, dix ans de suite, attaqué du mème Aecidium. J'ai v'u un pied de cet Erythronium attaqué de son Aecidium, qu'on avait transporté avec sa motte à un quart de lieue de distance dans une orangerie; l'annce suivante les nouvelles feuilles de cette plante ćtaient attaquées d'Aecidium comme celles de l'annće précédente. - 2.0 Il me parait prouvé par l'observation que les champignons parasites ont chaquc annéc une époque fixe; que ccux de cette annce ne peuvent provenir des graines disséminées actrellement par d'autres individus, mais des graines de l’amnée précédente. Il faut en effet leur laisser le temps de croitre: or cette croissance n'est pas rapide, et tous ccux dont j'ai eu l'occasion de suivre l'histoire sont resté plusieurs mois pour parvenir à leur maturité. On sait d'ailleurs qu'on n'est point cncore parvenu, en saupoudrant une plante de la poussicre de son parasite, à faire développer ce parasite, quoiquc cette cxpérience ait été tentéc plusicurs fois sur les parasites du froment, et que je l'aic tenté pour quclques autres. Or, si ces faits sont admis, ils s'expliquent bien plus facilement par la théoric, que je propose, que par celle indiquée par Sir Banks. On conçoil que 
les graines de champignons se conservent bien mieux déposées en terre, que voltigeant dans l'air."

"De toutes ces considérations, je suis, ce me semble, autorisé à conclure, que si, cornme personne n'en doute, ces parasites sont des végétaux, qui se reproduisent de graine, si l'introduction de ces graines ne peut avoir lieu que par les pores corticaux ou par ceux des racines, c'est à cette dernière voie qu'on doit donner la préférence. Il m'a paru même que le petit nombre d'objections qu'on peut faire à cette théorie, sont communes à l'une et à l'autre. Ainsi, par exemple, l'action du chaulage pour détruire l'Uredo caries, paraịt contraire aux idées que je viens d'énoncer; mais je remarquerai que l'Uredo caries (Carie, Kornfrass) s'écarte sous plusieurs rapports des habitudes communes à tous les Urédo. Au lieu d'attaquer les feuilles, il s'établit de préférence sur les glumelles et surtout sur les graines des graminées. Ils est probable que les sé-

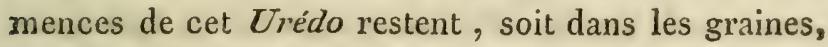
soit peut-être fixées à la surface; qu'elles sont ainsi transportées par les semailles; que les chaulage détruit celles de ces sémences, qui sont fixées à la surface des grains du blé : mais que si cette opération parait ne pas réussir constamment, c'est. qu'elle n'a aucune action sur les graines l'Urédo, qui peuvent se trouver dans la terre où le blé a été semé."

"Peut-être même pourrait-on déja, au moyen des vues que je viens de présenter, indiquer quel- 
que procédé pour diminuer les ravages de ces parasites; et ce moyen sera une confirmation de la vraie théorie des assolements. Lorsqu'un champ de blé a été fort attaqué par l'Uredo carbo, ou par la Puccinia graminis, si l'année suivante on y resème ou du froment, ou quelqu'autre graminée, cette nouvelle moisson en sera infestée comme la première; mais si au contraire on y place des végétaux d'une autre famille, les graines de l'Uredo carbo y seront, il est vrai, introduites par la sève, mais n'y trouveront pas la nourriture, qui leur convient; elles avorteront sans produire de dommage, et le terrain s'en trouvera dépouillé. Je livre cette idée aux agriculteurs, pour que des expériences faites en grand la vérifient ou la condamnent."

Cette théorie très-ingénieuse, et que je doute fort que des faits avérés puissent détruire, explique trèsclairement ce que sont les maladies des blés et détruit en même temps quelques idées bizarres sur la cause de la rouille (Uredo rubigo-vera), que quelques personnes avaient voulu attribuer au pollen du Berberis vulgaris, d'autres à l'Aecidium berberidis. Il serait bien difficile de faire comprendre comment le pollen du Berberis pourrait seul produire un champignon, ou comment les graines d'un Aecidium pourraient produire un Uredo. Avec un raisonnement parcil à celui que l'on a avancé, on pourra bientôt dire que l'on a semé des Pommes-de-terre ct qu'il a cru des Rosiers. Que de miliers de pieds de joli Berberis vulgaris ont péri par cette ridicule théorie, que les idées de Mr. DeCandolle, trop 
peu conues, détruiront j'espère complettement si l'on veut les apprécier à leur juste valeur.

\section{UREDO CARBO. $\left({ }^{*}\right)$ \\ Charbox, Nielle, (Brand.)}

Cespitulis maximis irregularibus, fuscis seu nigris, organa fructificationis occupantibus; capsulis globosis parvulis. DeC. Syn. n. ${ }^{0} 615$.

Cet Uredo carbo est composé d'une poussière noire toujours bien visible à l'extérieur de l'épi, et qui détruit et désorganise les parties de la fleur ou du fruit : Cette poussière, vue au microscope, parait composée de globules sphériques fort-petits et absolument dépourvus de pédicelle. Ces globules sont souvent comme collés les uns aux 'autres, de manière à paraître de petits filaments en chapelet. Ce n'est qu'avec la lentille n. ${ }^{0} 1$. du microscope de Dellebare, quion peut bien distinguer la forme de ces globules. La poussière du charbon se répand avec facilité, et n'a point, même lorsqu'elle est fraiche, de mauvaise odeur. Elle nuit aux Céréales, parce qu'elle diminue la quantité de la récolte, mais comme elle se disperse avec la mois-

$\left(^{*}\right)$ Uredo. Peridium inullum; capsulae sessiles, unilo. culares. Fungilli caespitosi, sub epidermide foliorum et caulium herbacearum orti et epidermide ruptifacile dis. persi. DeC. Syn. 47. 
son, elle ne nuit pas à la qualité de la farine. Outre les Céréales citées ci-dessous, le Charbon attaque un grand nombre de graminées sauvages."

28. A. - Uredo carbo hordeI.

Ustilago J. Bauh. Hist. 2. p. 418. fig. 1. et 2.

UREDo Segetum hordei, pseudo-peridio subelliptico, ruguloso; pulvere latente. Pers. Syn. fung. p. 224. - DeC. Fl. fr. 2. n.0 615. var. a - Syn.n.0 615. - Encycl. Bot. 8. p. 227. - Tess. Mal. des grains, p. 306. f. 2-4.

Uredo carbo hordei DeC. Fl. fr. 5. $n 0^{0} 615$. p. 76.

Fig. J. Bauh. Hist. 2. p. 418. f. 1. ct 2.

Tess. Mal. des gr. f. $2-4$.

Exs. Mougeot et Nestl. Stirpcs. 3. n. ${ }^{0}$ 291. (Ured. segetum.)

Ser. Herb. cer. 28. A.

Je n'ai encore trouvé le charbon de l'Orge que sur l'Hordeum hexastichon, vulgare et distichon, var. A., mais lorsqu'on cultivera ausśi les autres espèces ou variétés très en grand, on l'y retrouvera probablement aussi. L'épi charboné se remarque dès sa sortie de la gaîne, et l'épi, à l'entier développement de ce parasite, en est le plus souvent entièrement rongé et réduit au seul rachis.

Franc. Charbon de l'Orge. 
28. B.-URedo carbo triticr.

Lycoperdon tritici $C$. Bierk. Act. suec, ann. 1775 .

Unedo segetuir tritici, subeffusa. Pers. Syn. fung. p. 224. - Chantrans, Recherches chimiq. et microscop. sur les conferves etc., n. ${ }^{0} 28 . f .28 .-$ DeC. Fl. fr. 2. n. ${ }^{0}$ 615. var. b.

Uredo carbo tritici DeC. Fl. fr. 5. n.0 615. p. 76 .

Fig. Chantr. conf. fig. 28.

Il est heureusement fort-difficile de trouver en Suisse le Charbon du blé, peut-être ce champignon attaque-t-il moins fréquemment les épeautres, si abondamment cultivés dans la partie allemande de la Suisse. Les cultivateurs, dont les moissons seraient infestées de cette variété du charbon, m’obligeraient bcaucoup en m'en envoyant quelques cents exemplaires.

Franc. Charbon du blé.

\section{C. - Uredo carbo avenae.}

Reticularia segetum Bull. Hist. des Champign. 1. p. 90. T. 472. f. 2 .

Uredo segetum avenae, eflusa, fluctuans. Pers. Syn. fung. p. 224. Chantr. conferv. n.0 54. T. 54 . - DeC. Fl.fr. 2. n. ${ }^{0}$ 615. var. c. -

Uredo carbo avenae DeC.Fl.fr. 5. n. ${ }^{0} 615 . p .76$. 
Fig. Bull. Champign. 1. T. 472. f. 2.

Chantr. conferv. T. 54.

Exs. Ser. Herb. cer. 28. C.

L'Aveza sativa est souvent attaquée du charbon; quoique l'Avena orientalis soit fréquemment cultivée autour de Berne, je ne l'y ai pas encore remarqué:

Franc. Charbon de l'avoine.

Ce sont les trois seuls genres de Céréales que j’aie vus en Suisse attaqués du charbon. Le genre Panicum en est aussi quelquefois affecté, mais je ne l'ai jamais trouvé. Cet Urédo se développe souvent aussi sur différentes graminées sauvages et diffère fort peu de l'Uredo urceolorum.

\section{UREDO MAYADIS.}

Uredo segetum mays zeae DeC. Syn. Fl. gall. n. ${ }^{0}$ 615. - Encycl. Bot. 8. p. 227. = Charbon du Maïs Bosc Dict. agr. 3. p. 339. - Tillet Mém. acad. Paris 1760. p. 254. Imhof. Diss. in 4.0 Argentor. 1784.; ex Bibl. Banks 3. p. 431. - Carrad. Diss. in Giorn. pisan. 7. p. 301. et 10. p. 265.

Uredo maYadis DeC. Fl. fr. $5 . n 0^{0}$ 615. a. p. 77.

Je n'oserais pas affirmer d'une manière bien positive que l'Urédo du Maïs soit une espèce distincte du charbon; mais il présente des phénomènes si différents dans sa végétation, que j’ai peine à croirc 
à leur identité. Il attaque tantot la tige à l'aisselle des feuilles, tantot les fleurs mâles, tantot les graines mêmes du Maïs. La partie attaquée grossit et prend la forme d'une tumeur, d'abord charnue, puis entièrement remplie d'une poussière noirâtre, inodore et très-abondante. Ces tumeurs ont depuis la grosseur d'un pois ou d'une noisette, lorsqu'elles attaquent les fleurs mâles, jusqu'à celle du poing et au-delà, lorsqu'clles attaquent la tige, et même le grain. Lorsqu'elles sont parvenues à maturité, l'épiderme, qui les recouvrait, se rompt au moindre choc et laisse échapper la poussière. Cette plante est donc intermédiaire entre le charbon et la carie; sa poussière, comme celle du charbon, est inodore, composée de globules fort-petits : comme celle de la carie, elle nait à l'intérieur des grains, pour se répandre ensuite au déhors. On trouve cette maladie dans tous les champs de Maïs situés dans les lieux humides et arrosés, et surtout dans les années pluvieuses."

Franc. Urédo du Maïs.

\section{UREDO C A R I E S.}

\section{CARIE (Kornfrass, Kornfäule.)}

Carie Tessier. Mal. des grains, p. 217-294. (fig.) - Prevost. Diss. Montaub. 1807. (fig.) -

U. CARIES DeC. Fl. fr. vol. 5. n. ${ }^{0} 615$. b. p. 78. La carie n'attaque que les blés, soit les 
froments, soit les épeaulres; elle nait dans l'intérieur même du grain, qu'elle ne déforme presque. point, mais qu'elle change en une poudre noirâtre, fétide lorsqu'elle est fraiche et qui ne se répand point d'elle-même au déhors. Cette poussière, vue au microscope, est composée de globules deux fois plus gros que ceux du charbon, peu adhérents les uns aux autres, et dépourvus de pédicelles. Mr. Prévost a remarqué que ces globules, mis dans l'eau, y poussent des radicules; les épis cariés se distinguent à peine des épis sains, et n’ont le plus souvent qu'une partie des grains qui soit attaquée. Cette poussière persiste dans le grain récolté et altère la qualité de la farine; elle est très-contagieuse, et quelques grains de blé carié suffisent pour se répandre sur les graines saines, et pour que les plantes qui en proviennent soient cariées. Le chaulage est le seul moyen connu d'empêcher la propagation de ce champignon, qui, de l'opinion de tous les agriculteurs, s'introduit par les racines, et s'élève jusqu'à l'épi, par l'intérieur même de la plante." Pour acquérir cette conviction, peut-être pourrait-on développer la carie dans des graines, en jetant la poussière de cette carie dans une petíte partie de terrain ensemencé de graines saines, et voir si on parviendrait à obtenir dans cette portion de terrain ensemencée de l'Uredo caries, des épis où se serait développé ce champignon.

Il résulte des expériences faites par MM. Fourcroy et Vauquelin, (Mémoires de l'Institut, scienc. physiq., vol. 6. p. 514-530.) que lo blé carié ne 
contient plus ni gluten, ni amidon, ni matière sucrée, et que toutes ces substances sont réduites à l'état d'un corps huileux et charboneux, à peu près comme les bitumes noirs.

\section{UREDO RUBIGO - VERA.}

\section{RouItLe (Rost.)}

Routlie Tess. Mal.des grains, p. 200-215. ( fig.)

Uredo rubigo-vera DeC. Fl. fr. $5 . n .^{0}$ 623. $d$. p. 83.

"La Rouille des agricultcurs est un Urédo trèsdistinct, par sa forme et son apparence, qui a été quelquefois confondu avec la Puccinia graminis dans sa jeunesse, mais qui en est entièrement distincte; elle nait sur la surface supćrieure des feuilles, et plus rarement sur la surface inférieure, sur la gaine des feuilles, ou sur la tige des graminées, et principalement du Triticum vulgare; elle y forme des pustales ovales, extraordinairement petites, mais ordinairement très-nombreuses; dans leur jeunesse elles sont recouvertes par l'ćpiderme, et offrent alors l'apparence de petites taches, à peine proéminentes; ensuite l'épiderme se rompt par une fente longitudinale, et laisse voir une poussière jaune: enfin cette poussière devient rousse, mais jamais noire; elle s'envole facilement et laisse les feuilles mouchetées de petits points roussâtres. Cette poussière, vue au microscope présente depuis sa nais- 
sance jusqu'à sa mort, des capsules ovoïdes, presque sphériques, très - petites, dépourvues de pédicelle. Il arrive quelquefois que sur les mêmes pieds, qui portent la rouille, on trouve là Puccinia, ou la Sphaeria punctiformis graminaria, mais ces plantes parasites, quoique mêlées quelquefois ensemble, se distinguent sans peine. Lorsque la rouille est abondante, elle épuise les graminées qu'elle attaque, au point de diminuer les récoltes d'une manière marquée. Le Secale cereale, (Ser. Herb. cer. 31. A.) sur lequel j'ai pris mes exempl., n'avait pas monté en épi, probablement par l'épuisement qu'avait produit la rouille; l'Agrostis alba était dans le même état. (Ser. Herb. cer. 31. B.)

L'Uredo caricina DeC. Fl. fr. 5. n. ${ }^{0} 623$. e. , que $\mathrm{Mr}$. Schleicher a trouvé à la surface inférieure des feuilles du Carex pseudo-cyperus, et que j'ai retrouvé sur le Carex sylvatica, (qui n'était pas monté en épi) ressemble tellcment à l'Uredo rubigo-vera, qu'on pourrait facilement croire, qu'il n'en est qu'une simple variété, due au lieu où la plante a crû. Il en diffère par sa couleur, rousse dès sa naissance, et qui devient ensuite brume cn vieillissant; ses pustules sont éparses, ovales, très-petites, bordées par les débris de l'épiderme rompu; ct les capsules sont sphériques. La Synonymie de cet Uredo caricina est: $U$. caricis Schl. Cypt. exs. cent. 2. n. ${ }^{0}$ 92. - DeC. Syn. n. ${ }^{0} 624 .{ }^{*}$ - Encycl. Bot. 8. p. 230. n. ${ }^{0} 46$. - Unedo caricina. DcC. Fl. fr. 5. n. ${ }^{0}$ 623. c. p. 83. Ser. Herl. cer. 31. * (Caricis sylvatica.) 


\section{3}

\section{SCLEROTIUM CLAVUS. (*)}

\section{ERgot. Cravus. (Mutterkorn.)}

S. corniforme cylindraceum sulco longitudinali interdum notatum, intus album. extus purpureonigrum. DeC. Mem. du Mus. 2. p. 416.

Scl. clavus DeC. Fl. fr. 5. p. 115. Mém. du Mus. 2. p. 416. $f$. 3. = Clavis siliginis Lonic. ex. C. Bauh. = Clavus Bibl. Banks 3. p. 429. = Secate luxurians $C$. Bauh. Pin. 23. n.0 4. $-J$. Bauh. Hist. 2. p. 417. = Secale cornutum Baldinger Diss. Jena 1771. - Nebel Diss. dua, Giessa 1771 el 1772. = Secalis Mater Thal. herc. p. 47. = Grana secalis degenerati Brunner in Ephem. cur. nat. dec. 3. ann. 2. p.348. = Seigle ergoté Dod. Mem. acad. scienc. 10. p. 561. - Salerne, Mem. sav. étrang. ac. Paris 2. p. 155. - Foug. de Bond. Mem. acad. sci. Paris. 1683. p. 101. Bull. herb. t. 3. = ERGoT Tissot in phil. trans. 55. p. 106. - Rosier Journ. Phys. 4. p. 41. Parmentier Journ. Pliys. 4. p. 144. - Tessier Mém. Soc. roy. médéc. 1776. p. 417.; 177\%. p. 587. - Mal. des grains, p. 21. add. 18s. f. 1-5.; p. 189. f. 1-6. - Bosc Nouv. Dict. agric. 5. p. 261. Plench. Pathol. trad. 185. = Granosprone Re

(*) Sclerotium, forma varium; cortex dura; caro plus minusve solida (vero similiter seminifera) venis destituta. DeC. Syn. p. 58. - Fungus farctus, forma varius, intus laeve similare, externe in nonnullis demum corrugatum. DeC. Mém. du Mus. d'hist. natur. 1\$15.p.411.. 
malat. delle piante, 389. = MUTterkoRN Sohleger Diss. Cassel 1770. - Hernes inn. Schrift. Beritin. Ges. naturf. Fr. 1. Band. p. 244.

„L'ergot est une production qui a la forme d'une corne, et qui sort d'entre les glumelles des graminées, à la place où devait naître le grain. Il est à peu près cylindrique, long de six à dix lignes, souvent marqué d'un côté par un sillon longitudinal, obtus à son sommet, le plus ordinairement un peu courbé, blanc à l'intérieur, d'un bleu tirant sur le pourpre en déhors. L'ergot est très commun sur le seigle, dont il infeste quelquefois les moissons; d'ailleurs je ne l'ai trouvé en Suisse que sur plusieurs espèces du genre Bromus. On a beaucoup disputé sur la nature de l'ergot: On le regarde généralement comme une altération du grain, produite par un défaut de fécondation, ou par la piqûre de quelque insecte, ou par l'humidité. Sa grande analogie avec la plupart des Sclerotiums fait penser à Mr. DeCandolle que l'ergot est une espèce de ce genre, qui se développe sur l'ovaire, détruit le grain, et végéte à sa place."

L'analyse chimique du Sclerolium clavus DeC. faite par M. Vauquelin, comparativement au Scl. stercorarium. (Mém. du Mus. d'hist. natur. 1817. p. 198.) prouve que ces deux substances sont assez différentes. 1.0 Que l'infusion du Sclerot. stercorarium est sans couleur, (tandis que l'ergot en donne une d'un rouge jaunàtre, qui s'applique mieux sur la laine que sur la soie), sans acide, qu'elle est pré- 
cipitée plus abondamment par l'alcool, la noix-degalle et le chlore; qu'clle est beaucoup plus mucilagineuse que celle de l'ergot; que son extrait n'a pas la saveur désagréable et acre de celle de l'extrait de l'ergot, au contraire il est doux et mucilagineux comme celui des champignons comestibles. $-2 .{ }^{\circ} \mathrm{Le}$ Sclerot. stercorarium soumis à la distillation à feu nu, ne donne pas d'huile épaisse et butireuse comme l'ergot, l'air du récipient est alcalin comme celui de l'ergot; mais le produit liquide est beaucoup plus acide et moins épais. - $3.0^{\circ}$ L'ergot contient une huile fixe toute développée, qu'on peut extraire par la simple pression; tandis que le Sclerotium stercorarium n'en contient pas. Il y a encore dans l'ergot une espèce de résine très-acre, qui n'existe pas dans le Sclerot. stercorarium. Enfin l'ergot renferme de l'ammoniaque tonte fermée, qui s'en dégage à la température de l'eau bouillante, et le Scl. stercorarium n'en donne qu'à une chaleur rouge. - D'ailleurs les grains de seigle ergotés ne contiennent presque plus de traces sensibles de leur matière amylacée, qui a été remplacée par une sorte de matière muqucuse, et le gluten parait s'y trouver aussi transformé en une huile épaisse et en ammoniaque.

On a accusé l'ergot du seigle de produire une espèce de gangrène, appelée Ergotisme. Son âcreté pourrait bien donner naissance à cette maladie, mais il serait aussi possible que l'humidité, et surtout la disette qui règne plus ou moins dans les années pluvieuses, en fut la cause. Si l'ergot nuit 
aux animaux, ce ne peut guère être qu'à la longue, car donné à grande dose à des lapins il n'a produit aucun effet sensible. (Dr. Mayer.)

\section{PUCCINIA GRAMINIS. (*)}

$P$. cespitulis è luteo fuscis et nigris sparsis linearibus parallelis, stipite brevi, capsula biloculari subclavata, loculo ultimo majori. DeC. Syn. $n 0^{0}$ 596.

Puccinia graminis Pers. Syn. 228. - Hediw.f. fung. ined. T. 6. - DeC. Fl. fr. 2. ${ }^{0}{ }^{0}$ 596. Syn. n. ${ }^{0}$ 596. p. 46. Fl. fr. 5. p. 59. n. ${ }^{0}$ 596. var.a.

Exs. Ser. Herb. cer. 33. A.

La Puccinie des graminées, souvent nommée noir par les agriculteurs, se dćveloppe sur toutes les parties des graminées et plus particulièrement sur les orges et les avoines, plus rarement sur les blés. Elle y forme des pustules ovales ou linéaires, brunes à leur naissance, puis très-noires. Elles soulèvent d'abord l'épiderme, lc fendent en long, et

(*) Puccinia. Peridium nullum; capsulo uni aut mula tiloculares, apice dehiscentes, pedicellate, disco sub= carnoso insertce. Fungilli fusci aut nigri in cespitu= los aggregati, in foliis vivis parcasitici. DeC. Syn. p. 44. = Micheli avait donné ce nom de Puccinia à plusieurs espèces de Gymnosporrngitums en l'honneur de Thomas Puccini, prof. d'anatomie a Florence. DeC. 
restent bordées de ses débris. Chaque pustule, vue au microscope, présente un amas de petites plantules, dont le pédicelle est blanc, transparent, cylindrique; la capsule est alongée en forme de massue, à deux loges séparées par une cloison, mais sans étranglement prononcé; la loge inférieure est un peu en cône renversé, la supérieure est arrondie et la superficie de cette capsule est lisse. - La var. b. de cette Puccinie ( $P$. arundinacea Hedw. fil. Fung. ined. T.7. DeC. Encycl. Bot. 8. p. 250. Ser. Herb. cer. 33. B. (junior.) et C. (senior.) qui croit sur les graminées dures et fermes (Arundo phragmices), y forme des pustules plus grosses et plus convexes. Cette plante parasite nuit peu à la maturation des blés; $\mathrm{j}$ 'ai vu des orges et des avoines, dont les gaìnes des feuilles et le chaume étaient couverts, n'avoir pas des épis moins beaux que les individus voisins, qui étaient très-sains.

On trouve encore assez souvent, quand on examine à la loupe les épis, de très-petits points noirs, qui recouvient les glumes, les glumelles, les arêtes, le chaume des céréales, et qui ne sont que la Spharia punctiformis var graminaria. DeC. Fl. fr. 5. p. 145. $n .^{0}$ S06., mais, qui sont si petits, qu'ils ne peuvent nuire à la plante, sur laquelle ils se trouvent. 


\section{USAGES ECONOMIQUES DES CÉRÉALES.}

\section{PARTIE.}

Toutes les céréales ont besoin de préparations préalables pour pouvoir servir à nos besoins. Une fois coupées elles sont liées en gerbes, et subissent dans nos granges une première préparation; les unes tombent nues sous le fléau, c'est-à-dire, n'ayant que deux pellicules très-minces, formant le gros et le petit son, qui recouvrent la partie vraiment nutritive, et alors on dit que les graines tombent nucs sous le fléau, (blé à graines nues, froments des Français, FRUmIENTA); ou bien ces blés restent enveloppés de la glumelle, appelée communément bourre, par la fragilité de leur rachis ou axe de l'épi, et on les nomme blé à graines enveloppées. (Froments des Suisses allemands, épeautres, Speltae.) Les vrais froments, particulièrement cultivés dans les pays méridionaux, passent entre deux meules très-rapprochées, et sont réduits en farine, qui est aussitot séparée du son par des blutoirs. $\left(^{*}\right)$ Les épeautres au contraire, principalement cultivés dans les pays du Nord, ont besoin d'être privés de la glumelle, ou bourre, qui les renferme étroitement, ou autrement dit, subissent préalablement l'opération du débourrage. Le grain passe entre deux meules écartées,

(*) Espèce de tamis formés en tissu de laine et dispo. sés en longs sacs étroits. 
tées, sort de sa bourre et en est aussitot séparé; $\left({ }^{*}\right)$ alors il est réduit en farine par le même procédé que les froments.

\section{F A R I N E.}

Les froments se réduisent généralement plus vite en farine que les épeautres. Les premiers en général empâtent davantage les meules. Des blutoirs, disposés convenablement, séparent le son de la farine, qui elle-même passant par d'autres blutoirs plus ou moins fins, se sépare en deux portions, l'une appelée fleur de farine, (Ser. Herb. cer. 34. A.) dont on fait le pain blanc, et l'autre est la farine ordinaire, (Ser. Herb. cer. 34. B.) Cette farine de blé est formée de deux substances, $10^{0}$ d'une matière féculente, qui se précipite par le lavage, dont est fait l'amidon, qui, mèlé arec l'eau, s'y dissout à la température de 50 dégrés, pour former l'empois, et $2 .^{\circ}$ d'une matière fibreuse, tenace, insoluble dans l'eau, qui est le gluten, lequel est composé de beaucoup d'ammoniaque, et de plusieurs autres substances. Les farines des blés paraissent contenir plus de gluten que celles d'orge, de seigle et d'avoine,

(*) Cette bourre, mêlée avec du son, sert à nourrir les chevaux et à emballer les objets fragiles, à fire des paillasses d'enfants, ou est mise en litière, puis réduite en fumier. - Les épeautres sont exposés en vente, soit revêtus, soit dégamis de cette bourre; mais ils sont toujours semés avec la borrre, même lorsqu'on se sert des semoirs. 
qui semblent essentiellement formées de matière amilacée. Il parait que les froments contiennent plus de gluten que les épeautres, et c'est probablement à cause de la ténacité du gluten, que les boulangers de la partie allemande de la Suisse trouvent le pétrissage de la farine des froments plus difficile que celui des épeautres, qui contiennent proportionnellement beaucoup plus de matière amilacée. Je présume que le Triticum amyleum, dont la farine est extrêmement blanche et pure, ct dont les amidoniers de l'Aargovie font leur bel amidon, contient moins de matière glutineuse que tous les autres blés. La farine de seigle, quoique presque entièrement formée de cette matière amilacée, ne produit probablement un pain si bis que par son mélange avec une matière extractive colorante. L'orge contient, outre une grande quantité de fécule ou amidon, une matière sucrée, qui facilite sans doute la fermentation spiritucuse, et ontre cela une matière acide, qui occupe particulièrement ou la glumelle, ou le péricarpe et périsperme (son), car il ne se trouve pas dans l'orge grué ou privé de la glumelle et de ses enveloppes.

La farine de Maïs est jaune, (Ser. Hcrb. cer. 34. C.) celle du Millet, Panicum miliaceum et italicum est plus pâle, et celle du Triticum durum (Ser. Herb. cer. 34. D.) est d'un blanc jaunâtre.

Les eaux-de-vie de grains doivent leur existence à la fermentation vineuse, que l'on fait subir aux graines céréales. Ces eaux-de-vie acquièrent un 
gout désagréable par 'une matière huileuse, qui s'élève dans la distillation.

Le riz est une graine essentiellement amilacée, qui ne contient que des traces à peine perceptibles de gluten et de phosphate de chaux. Elle diffère done des autres graines céréales, servant à la nourriture de l'homme et des animaux, lesquelles renferment beaucoup de ces deux matières; ainsi le mode, suivant lequel le riz nourrit, doit être différent de celui du froment. Nous avons fait tous nos eflorts pour découvrir la matière sucrée dans le riz, mais ils ont été sans succès; il est cependant singulier, que cette graine ne contienne pas le corps sucré, car l'on assure que dans certains pays on cn retire de l'eau-de-vie, qui est appellée Rack. - Au reste la pomme de terre, qui ne contient pas non plus de sucre, fournit cependant de l'eau-devie, soit qu'on l'employe crue, soit qu'on la fasse cuire pour la faire fermenter. - De là il faut conclure, ou qu'il y a autre chose que le sucre, qui peut former de l'alcool, ou que le sucre se trouve quelquefois tellement enveloppé dans les végétaux, qu'il échappe aux moyens de la chimie. (Vauquelin, Analyse du riz, mémoires du Muséum d' Histoire naturelle 3. p. 229. (1817.)

\section{S O N. (Kleyen.)}

Le son des céréales n'est autre chose que les deux enveloppes propres à la graine. La plus extérieure ou péricarpe forme le gros son, grobe Kleyen, 
(Ser. Herb. cer. 35. A.) la pellicule intérieure ou périsperme, enveloppant immédiatement la partie farineuse forme le petit son, feine Kleyen, (Ser. Herb. cer. 35. B.) Ces deux substances mêlées ensemble sont séparées de la farine par les blutoirs; puis le tamis laisse passer le petit son et reticnt le sros.

Ces sons repassés entre les meules, et séparés encore de la farine, surtout le petit son, contiennent encore de petites portions de matière féculente, et peuvent conséquemment servir à la nourriture de quelques animaux. Le petit son sert, surtout dans quelques endroits, à saupoudrer le pain noir avant de l'enfourner. L'amidonier en retire aussi de l'amidon; on s'en sert pour former les pelotes, il entre dans les bains émolliens, etc. etc.

\section{G $\quad R \quad U \quad A \quad U \quad X$.}

On entend ordinairement par gr'uer ou monder, l'action de priver les céréales de lcur's enveloppes propres ou sons, mais quelquefois on lcur fait subir un léger ćcrasement. On grue le plus grand nombre sans préparations prćliminaires; une seule est lessivée et torréfiće avant d'être gruéc, c’est l'avoine. Je vais tàcher de donner une idée de ces différentes espèces de gruaux, dont on trouvera toujour's des échantillons dans l'Herbicr crivial. 
GRUA U D'ÉPEAUTRE.

Semoule, Simola. - Gries. - (Ser.Herb.cer. 36. A.)

Le Simola se fait en Suisse aver l'épeautre. Au premier écrasement, l'épeautre débourré se réduit en petits grains secs, et une partie en farine, qui en est séparée par les blutoirs. Ces petits grains ou Simola (en allemand Gries) sont livrés au commerce, ou le meunier les fait repasser entre les meules pour les écraser, et il en retire la plus belle farine.

Ce Simola est plus cher que la farine. On en fait un grand usage en Suisse pour les soupes, les bouillies. Il cuit très-vite et est d'une très-facile digestion. C'est cette même préparation, qui, coloriée, forme les anthères des flcurs artificielles, et que les fabricants nomment improprement: graines. (Ser. Herb. cer. 36. B.)

\section{GRUAU DE BLÉ-CORNÉ. (T. durum.)}

Simola de Gênes. (Ser. Herb. cer. 36. C.)

Ge gruau est dû au premier écrasement du Triticum durum, dont la substance se brise en petits morceaux anguleux. Je ne pourrais mieux le comparer qu'à la gomme arabique finement concassée, il en a la couleur et presque la transparence. Il cuit aussı vite que celui d'épeautre, et est d'un gout beaucoup plus délicat. 
GRUA U D O R G E.

Orge mondé. - Graupen. - (Ser. Herb. cer. 37. A.)

Les paysans mondent ordinairement l'orge pour s'en servir comme aliment. C'est sous une meule à faire l'huile, qui tourne en cercle dans une auge circulaire, qu'ils le préparent pour leurs besoins, et ce n'est que dans cet état qu'on le trouve au marché. Mais il est mal préparé, et une partie de la glumelle, (ou paille) si adhérente dans les graines d'orge, s'y trouve encore fixée, ce qui le rend fort-désagréable lorsqu'il est cuit.

L'orge entière, bouillie dans l'eau, forme une tisanne rafraichissante, souvent employée en médecine; mais il est préférable de la préparer avec l'orge mondé ou l'orge perlé, car les enveloppes de la graine contiennent une substance acide, qui rend acre la première décoction.

\section{GRUAU D'ORGE-PERLÉ.}

Orge perlé, Orge d'Ulm. - Ulmergerste, Perlgerste.

(Ser. Herb. cer. 37. B.)

L'orge perlé, aussi connu sous le nom d'orge d'Ulm, ne se fabrique point dans le pays; il est privé de la paille, et des enveloppes de la graine 
par des moulin-rapes (*), qui en même temps en arrondissent les grains qui ressemblent en quelque sorte à des perles. On fait un grand usage en soupes de cette préparation dans la Suisse ; on l'a tirée jusqu’à présent de l'Allemagne, mais MM. Lacroix, négociants à Berne, ont fait construire un moulin pour la gruer, et les essais qu'ils ont faits, n'ont pas été sans succès.

\section{GRUAU DE MILLET.}

Millet grué. (Ser. Herb. cer. 3s.)

Ce gruau, dont les paysans suisses font un assez grand usage, est cuit dans du lait ou dans l'eau, et assaisonné dans ce dernier cas avec du sel et du beurre. Il fournit un aliment sain et agréable. Ils le préparent sous les meules à huile comme l'orge mondé du pays, et l'exposent souvent en vente au marché.

\section{GR U A U D'A V O I N E.}

(Ser. Herb. cer. 39.)

L'avoine est aussi gruée comme les autres céréales, ou plutot débourrée; on n'enlève par cette

(*) Ces moulins n'ont qu'une meule horizontale, et qui est toujours en mouvement; les parois qui l'entourent sont en bois et hérissées de milliers de pointes de fer. La meule met toujours en mouvement le grain que les dents de fer des parois déchirent, et qui d'ailleurs s'arrondit par le frottement circulaire. 
opération que les deux valves de la glumclle, qui entourent étroitement la graine, sans la priver de ses deux tuniques, péricarpe et périsperme, qui dans les blés forment le gros et le petit son, car souvent on retrouve sur sa surface une partie des petits poils couchés qui la recouvrent.

Cette préparation n'est employée dans le pays que pour nourrir des oiseaux - de - volière, et quelquefois pour en faire des tisanes, mais nullement comme nourriture.

\section{GRUAU D'AVOINE TORRÉFIÉ.}

Haberkern, Habermehl. (Ser. Herb. cer. 40.A. B.)

Le gruau d'avoine torréfié, nommé dans le pays Haberkern et Habermehl, se prépare indistinctement avec toutes les variétés blanches de l'Avena sativa et orientalis. Mr. Schnyder, architecte distingué de cette ville, en a formé depuis dix-sept ans un grand établissement et c'est à lui que je suis redevable de tous les renseignements sur la préparation du gruau d'avoine torréfié.

Il choisit l'avoine la plus lourde, la fait mettre dans une grande cuve carrée, percée au bas de l'une de ses faces d'un trou bouché par un bondon. On verse de l'eau bouillante sur cette avoine jusqu'à ce qu'elle dépasse l'avoine, puis on laisse couler l'eau, qu'on remet dans la chaudière, elle est ensuite verscé pour la seconde fois sur lavoine 
et on continne ainsi la lixiviation jusqu'à ce que les grains soient entièrement pénćtrés par l'eau, qu'ils soient réduits en mucilage transparent, et que l'on ne découvre plus de trace blanche an centre. Plus cette lixiviation approche de sa fin, plus l'eau devient foncće, et à la fin elle ressemble assez. à de la bière, du moins quant à la couleur. (*) On laisse entic̀rement couler l'eau, puis on fait glisser l'avoine toute mouillée par un trou pratiqué au milieu d'un four, qui se troure au-dessous, et qui est ensuite fermé par une pierre carrée. Le four, qui sert à la torréfaction de l'avoine, est grand, mais bas, et est chauffé rapidement à blanc par une large bouche avec du bois de sapin fendu en très-petits morceaux et très-sec. Quand le four est suffisamment chaud, on en retirc la braise, on le nétoye comme pour enfourner le pain, et on $y$ fait entrer l'avoine lessivée, qu'on a soin d'agiter souvent; sans cela les grains éclateraient et se réduiraient à rien. On réitère ensuite moins souvent cette agitation à mesure que le four se réfioidit; puis on eir ferme la bouche, que l'on rouvre de temps en temps pour répéter cette agitation. Quand l'avoine est à un dégré de torréfaction convenable, on la retire du four, puis on la met en tas dans un grenier ou dans des sacs. Elle peut se garder long-temps daus cet état.

On passe ensuite cette avoine lessivée et torréfiée entre les deux meules écartées, dont on se sert

(*) Cette eau contient un peu d'amilon dissout, et se putréfie très-facilement. On n'en fait aucun usage. 
pour débourrer l'épeautre, et dont la supérieure seule (ou courricr) est mobile; puis on tamise les graines ainsi privées de la bourre; celles qui sont bien entières forment le gruau de première qualité, ou Haberliern, et les graines plus ou moins brisées forment la seconde qualité ou Habermehl. Les portions trop petites et presque réduites en farine, sont séparées et cmployées cuites avec de l'eau, pour nourrir les veaux et pour engraisser les bestiaux. - Les chevaux mangent de préférence les glumes de l'avoine à celles de l'épeautre, et Mr. Schnyder les employe, surtout hachées, pour la nourriture des siens.

Il se fait une grande consommation de ce gruau d'avoine torréfié, il est envoyé dans toute la Suisse, même en Allemagne et en France. Cuit dans du bouillon, ou dans l'eau avec du sel et du beurre, il forme des soupes très-bonnes et fort numtritives, que les paysans assaisonnent assez généralement avec des feuilles hachées d'Allium schonoprasum (civette, grande ciboule, ou branlettes des Vaudois). Cette avoine, qui se réduit très-vite en une espèce de bouillie, a son péricarpe et périsperme si minces, qu'on ne les aperçoit pas lorsqu'elle est cuite, quoiqu'elle n'en ait point été privée, car on retrouve presque toujours des traces des poils couchćs, qui couvrent l'avoine débourrée.

Mr. Schnyder a aussi essayć de préparer de la même manière du gruau do blé et d'orge, sans avoir 
jamais pu réussir : Celui d'orge surtout avait un gout détestable, et on ne pouvait parvenir à le ramolir par la coction.

\section{PATES D'ITALIE. PATES DE GANES.}

On fait beaucoup moins en Suisse de pâtes alimentaires, qu'on prépare en Allemagne dans chaque ménage, probablement par la facilité qu'on a de se procurer des pâtes d'Italie. MM. les frères Lacroix en ont établi une grande manufacture à Berne, et ils ont bien voulu me donner tous les renscignements, qui ni étaient nécessaires.

Les Pâtes d'Italie se fabriquent avec une espèce de blé, très-rare en Suisse, mais qui y réussit bien, c'est le Triticum durum Desf. Nos blés suisses, mème l'épeautre et le blé amidonier (Trit. amyleum) sont trop farineux pour pouvoir servir à cet usaze. Ces négociants font donc venir d'Italie le blé corné, ( $T r$. durum) (*) le réduisent eux-mêmes en farine

( $)$ La grande majorité des grains appartient at Triti= cum durum; ils sont opaques, mais toujours cornés. $J$ 'en ai trouvé aussi quelques grains transparents, de la couleur de la gomme arabique, et parfaitement semblables à ceux du T. hordeiforme, que Mr. de Haller a bien voulu m'envoyer. Je persiste toujours dans l'idée que les $T$. durum et hordeiforme ne sont que la même plante; rien dans l'épi et dans les graines ne peut me les faire distinguer; mais le $T$, horo deiforme Host, de Mr. de Haller, a ses grains très. transparents. Mes variétés A. B. que je dois à $\mathbf{M r}$. le pasteur Steck, ont les graines opaqques. 
dans leur moulin, et en font leurs pâtes, qui sont très-belles. Il serait fort-intéressant d'avoir l'analyse de la farine de ce blé comparativement à celle du $T$. Spelta et amyleum. Ce $T$. durum est extrêmement différent des autres, c'est avec lui, comme je l'ai dit à l'article Simola de Gếnes, que ce Simola se prépare, et il a un tout autre aspect que le nôtre. On pourrait peut-être apprendre par cette analyse pourquoi cette farine est si sèche, et d'où lui viennent ses propriétés particulières; mais rercnons à notre objet.

On réduit la farine de blé corné en une pâte fort-épaisse, qu'on ne laisse pas fermenter, puis on la pousse avec force dans un cylindre de fonte d'un demi pied de diamètre, au fond duquel se trouve un disque de cuivre ćpais d'un demi pouce, percé d'une foule de trous coniques, dont la partie évasée est en haut; ces disques ont été faits en Italie, et sont fort-ingénieusement percés; les uns ont des trous presque capillaires sur la face inférieure des disques, et c'est celui avec lequel on fait le vernicelle, (Fadennudeln) qui tombe dans une corbeille placée audessous, que des enfants roulent en coquille et mettent sur des tablettes dans une chambre légèrement chauffée, où il se sèche aussitôt. La seule différence qui existe entre le vermicelle blanc ou ordinaire, (Ser. Herb. cer. 41. A.) et le vermicelle au safran ou jaune, (Ser. Herb. cer. 11. B.) c'est que, pour former la pâte du blanc, on ne prend que de l'eau, tandis que c'est de l'eau safranée, qui scrt à délayer la pâte du vermicelle au safran. Le disque ou filière, qui sert à préparer les pâtes plates, ou Lazaignes de Gênes, 
(Ser. Herb. cer. 41. C.) ou en forme de ruban, est troué dans le mème genre, mais au lieu d'avoir des trous ronds, ils sont percés de fentes en forme de coin, dont la partic la plus évasée est toujour's en haut, afin que la pâte s'y engage et y soit fortement pressée de haut en bas, et d'un côté à l'autre. Le distique des macaronis (Ser. Herb. cer. 41. D.) est percé d'après les mêmes principes, mais comme le gros cylindre de pâtes, qui passe par la filière, aurait eu ensuite trop de pcine à sécher, on a enfoncé dans chaque canal conique un crochet de laiton, qui forme le canal du macaroni en le fendant en même temps : par la force de la compression ce macaroni rapproche ses deux bords en sortant de la filière. On prépare encore à Gênes une autre pâte sèche, appelée Pâtes en graines, (Ser. Herb." cer. 41. E.) qui se fait avec la même farine, travaillée comme les pàtes précédentes; mais à mesure que le cylindre de pâte uni, ou diversement canelé, sort de la filière, il est coupé en petites tablettes minces par une lame continuellement en mouvement, de manière à former des petites tablettes circulaires ou étoilées, que l'on fait cuire dans le bouillon ou Ae toute autre manière.

\section{OSTIES. PAINS - $\dot{A}$ - CACHETER.}

Les osties et les pains-à-cacheter (improprement appelés en Suisse oublies), se font en délayant de la fleur de farine dans de l'eau, et en formant une pâte claire qu'on ne laisse pas lever, et que l'on fait 
cuire dans des plaques chauffées convenablement. Ces plaques de fer ou de laiton sont gravées pour les osties, et planes pour les pains à cacheter, dont la pâte est diversement coloriće. Lor'sque cette pàte est cuite, on en forme les pains-ì-cacheter avec des emporte-pièces de différentes grandeurs.

\section{P A I L L E S.}

Le chaume des céréales, particulièrement de quelques espèces de blés, est devenu un objet d'industrie d'une très-grande importance pour la Suisse. Il se fait des affaires énormes en tissus de paille aux marchés de l'Aargovic, ct depuis quinze ans dans ceux de Fribourg et de Bulle. Il n'est pas de paysan sur toute la route de Châtel-St.-Denis à Fribourg, qui ne s'occupe à tisser des pailles. Cette partie assez élevée du canton est plus riche en pàturages qu'en moissons, et l'on y trouve plus de céréales d'été que d'hiver. On devrait aussi tâcher de developper cette branche d'industrie dans le canton de Berne. Plusieurs paysans des basses vallées alpines de ce canton passent quelques mois de l'hiver presque sans rien faire: une partie, il est vrai, s'occupe à filer et à tisser ces étoffes bleues de laine, appclées dans le pays: Oberländertuch; mais des femmes, des enfans, soit pendant l'hiver, ou en gardant des troupeaux, pourraient tresser des pailles; et comme on prend de préférence les blés d'été pour cet objet, il serait facile de les élever cncore dans ces basses vallées; l'exemple et l'appat du gain répandraient aussi cette branche d'industrie 
dans les vallées supérieures, ensévelies sous la neige pendant quelques mois de l'année. Je crois bien avec Mr. Trog de Thun, que lors même que les blés pourraient réussix dans plusicurs vallées alpines, il ne faudrait pas transforner en moissons les parties planes des gras et riants pâturages de ces vallées; je crois, dis-je, que cette culture serait très-nuisible au pays, dont la vraie richesse consiste en pâturages et en vaches, qui nourrissent les habitants; mais il faut de bien petites places pour cultiver le blé nécessaire pour préparer la paille qu'une famille pourrait tisser dans une année.

J'ai dit ailleurs, qu'il n'y avait point de blés d'été et de blés d'hiver; je m'explique, je veux dire que les blés d'hiver ne présentent aucune différence botanique entr'eux, que ce sont les mêmes variétés, qui sont habituées plus ou moins à être sémées en blé d'hiver, ou en blé d'été, et qu'on parvient sans beaucoup de peine dans les pays, oir le temps de la végétation est assez long, à cultiver indistinctement une variété ou l'autre, et qu'elle r'éussirait également bien, si une ou deux années intermédiaires avaient pu, pour ainsi dire, leur en faire prendre l'habitude; je crois que tout agriculteur sans préjugé, et qui aura voulu me comprendre, sera de mon avis. Il n'y a point de doute que le blé semé en automne ne parcoure plus lentement les diverses périodes de sa végétation, et qu'il n'acquière plus de force, qu'il ne soit plus parfait; tandis que celui semé au printemps parcourt très-vite ces mêmes périodes, et qu'il est d'un tissu plus faible ; aussi prend-on, pour tisser, ces derniers, 
dont la paille est moins coriace, très-flexible et se travaille arec beaucoup de facilité,

Les petites portions de terrains, destinées par chaque paysan à la culture du froment pour la fabrication des chapeaux, sont ordinairement bien labourées, fortement fumées, et plantées en pommes de terre. Cette culture rend la terre très-meuble. Au printemps ils remuent encore la terre, déja bien ameublie, et $y$ sèment ordinairement le Triticum sativum, var. A. ( $T$. cestivum L.) ou bien d'autres variétés de ce même blé, en choisissant de préférence celles à épi blanc. A défaut de celles-ci ils prennent le T.vulgare compactum, et si ces variétés ne réussissent pas, ils se servent du même blé, mais semé en automne. Ils obtiennent par cette préparation du terrain de gros chaumes, dont les nœuds sont très-écartés, et les parois du chaume minces, flexibles et d'un bel éclat. Ils récoltent leur blé à la faucille un peu avant la maturité, en font des petites manipules de la grosseur du bras, et les mettent sécher devant ou autour de leurs maisons, afin qu'elles ne soient pas mouillées par la pluie. Ils coupent ensuite les tuyaux, qui ont quelquefois $5-11$ pouces de France de longueur, et battent les épis, dont les grains sont un peu ridés, n'étant pas entic̀rement murs, et desqucls on ne se sert jamais pour l'ensemencement.

BLAAN. 


\section{BLANCHIMENT DES PAILLES.}

Les tuyaux choisis sont liés par petites poignées et placés à leur entière dessication dans le soufroir, c'est un tonneau de sapin, placé ordinairement dans un coin de la cuisine, et d'environ trois ou quatre pieds de hauteur sur deux de diarnètre, défoncé par le bout supérieur, qui est fermé par un couvercle; une grille en sapin est établie vers le tiers inféricur: c'est là dessus que ce placent debout, les unes à côté des autres, plusieur's couches de petits faisceaux de paille sans nouds. Quand l'appareil est disposé, on allume du soufre dans un petit vase et on l'introduit par une petite ouverture en forme de porte, pratiquée au tiers inférieur et vide du tonneau; on referme cette petite porte, et la paille reste pendant vingt-quatre heures dans l'atmosphère sulfureuse. On la retire ensuite très-blanche et trèslustrée. (Ser. Herb. cer. 42. A.)

\section{DIVISTON DES PAILLES ET TISSAGE.}

La paille, ainsi préparée, est divisée avec l'instrument que réprésente la gravure. Ce fendoir est formé de 4,6,8 ou 10 tranchants, suivant qu'on veut avoir des lanières de paille plus ou moins larges. Quand elles sont ainsi divisées, (Ser. Herb. cer 42. B.) on les trempe dans de l'eau, et on les fait passer entre le doigt et un morceau de bois pour les rendre égales, et pour en émousser les bords, afin de les tresser ensuite de toutes sortes 
de manières. (Ŝer. Herb. cer. 42. C. D.) Ces pailles ainsi tissées sont formées en pièces d'une longueur déterminée, puis sont débourrées (c'est à dire, privés des petits morceaux de paille qui dépassent) puis roulées en pièces, passées pour la seconde fois au soufroir, et enfin exposées en vente. Alors cousues pour en faire des chapeaux.

Les femmes non contentes d'avoir de beaux chapeaux de paille, voulurent un moment les orner de fleurs en paille, et l'on en fabriqua. Pour cela on prend les plus gros tuyaux de paille, on les fend à un seul endroit, on les mouille, on les amincit le plus possible par leur face interne avec un couteau très-tranchant, puis ils sont collés sur de la batiste. On forme de cette manière des feuilles de paille, on en fait des piles en alternant avec une couche de papier et de paille; l'on met en presse, puis avec des emporte-pièces de toutes formes on enlève des portions de fleurs, de feuilles, qui sont ensuite montées en bouquets, par le moyen de petits fils de fer très-minces appelés carcasse.

Les chapeaux de paysannes de la majorité des cantons sont enduits, après avoir été cousus, d'une couche de soufre, qui les rend impénétrables à la pluie. (Ser. Herb. cer. 42. E.) Ceux des paysans sont ou blancs ou en paille noire sans être soufrés, et les habitants des villes en portent aussi en paille noire, tissés ou en tuyaux entiers, qui sont fortjolis et très-commodes.

Les négociants s'accordent assez généralement 


\section{$22 T$}

à dire, que les pailles dı canton de Fribourg sont les plus belles.

La paille de riz forme les plus beaux chapeaux que l'on puisse avoir; tous ceux que l'on vend en Suisse vienment de l'Italic, c'est là que les pailles sont tissées et cousues dans toute leur perfection. (Ser. Herb. cer. 42. F.)

D'ailleurs on fait ou l'on a fait de jolis petits outils de femmes, étuis, étuis d'aiguilles à tricoter, nates pour mettre sus et sous les tables, en un mot, nous avons mis à contribution les céréales de toutes les manières, et il serait bien difficile actuellement de les remplacer par d'autres végétaux.

\section{O M I S S I O N.}

La Famille des Graminées absorbait tellement ma pensée en travaillant cette Monographie, que j'ai oublié de faire mention du Polygonum fagopyrum L. (Blé noir, Sarazin, Carabin) $\left({ }^{*}\right)$, qui peut à la rigueur être considéré comme plante céréale, malgré qu'il appartienne à la famille des Polygonées. Il n'est guère cultivé que dans les cantons de Vaud et de Genève, et sert à la nourriture des hommes et des animaux.

(*) En allemand : Buchweizen. 


\section{CATALOGUE}

D. E S

\section{ESPECES ET VARIÉTÉS DES CÉRÉALES \\ CONTENUES DANS}

L'HERBARIUM CEREALE.

CErre collection des Blés, Seigle, Orges, Avoines, Maïs et Millets de la Suisse, est formée : $10^{\circ}$ de deux cartons divisés en 44 cases, qui contiennent des épis murs, et des pâtes d'Italie; $20^{\circ}$ d'un cahier' renfermant 25 exemplaires de céréales comprimées; 3. d'un carton, sur lequel sont fixées 9 capsules, contenant les maladies des céréales; $40^{\circ} \mathrm{d}$ 'un dernier carton où sont 21 petites capsules, dans lesquelles se trouvent les productions des céréales, comme farines, sons, gruaux et pailles. Le tout, soigneument arrangé, est renfermé dans un portefeuille in folio; (16 francs de Suisse, ou 24 francs de France.)

\section{Triticum vulgare. Vill.}

A. Spica laxa, aristata, alba, glabra. T'. cestivurn. I. (maturum.) Ser. Mél. 1.p. 87.

B. Spica laxa, aristata, alba, velutina. T. cestivum L. (mat.) Ser. Mél. 1.p. 89.

C. Spica laxa, aristata, rufa, glabra. T. costivumL. (mat.) Sex. Mél. 1.p. 89. 
D. Spica laxa, aristata, rufa, velutina. $T$. cestivum L. (mat.) Ser. Mél. 1.p. 90.

E. Spica laxa, mutica, alba, glabra. T. hybernum I. (mat.) Ser. Mél. 1. p. 90.

F. Spica laxa, mutica, alba, velutina. T. hy. bernzm L. (mat. et compr.) Ser. Mél.1.p.92.

G. Spica laxa, mutica, rufa, glabra. T. hybernum L. (mat.) Ser. Mél. 1. p. 93.

H. Spica laxa, mutica, rufa, velutina. T. hybernum L. (mat.) Ser. Mél. 1. p. 94.

I. Spica compacta, mutica, rufa, glabra. T. compactum Host. (mat.) Ser, Mél.1.p.95.

\section{Triticum. turgidum $\mathrm{L}$.}

B. Spica aristata, rufa, velutina. Pétanielle rousse. Dun, (mat.) Ser. Mél. 1. p. 98.

D. Spica aristata, nigra, velutina, densa. (mat.) Ser.Mél. 1.p.102.

3. 'Triticum durum. Desfont.

A. Spica aristata, vclutina. (mat.) Ser. Mél. 1.p. 107. B. Spica aristata, rufescente, glabra. T. hordeiforme Host. (mat.) Ser. Mél. 1. p. 108.

\section{Triticum Spelta. L.}

A. Spica aristata, alba, glabra. (mat.) Ser. Mél. 1. p. 118.

B. Spica aristata, alba, velutina. (comp.) Ser. Mé?. 1. p. 120 . 
C. Spica aristata, rufa, glabra. (mat.) Ser. Mél. 1. p. 120.

D. Spica mutica, alba, glabra. (mat. et compr.) Ser. Mél. 1. p. 120.

E. Spica mutica, rufa, glabra. (mat.) Ser. Mél. 1. p. 121.

F. Spica mutica, rufa, velutina. (mat.) Ser. Mél. 1. p. 222.

I. Spica exigua. (mat.) Ser. Mél. 1. p. 123.

\section{Triticum Amyleum. Ser.}

A. Spica aristata, alba, glabra; glumae mucrone incurvo. (mat. et compr.) Ser. Mél. 1. p. 125.

D. Spica aristata, atrata, villosa; seminibus obscuris. T. atratum Host. (mat.) Ser. Mél. 1. p. 129.

7. TRITICUM Monococcum. L.

A. Spica aristata, rufa, glabriuscula. (mat. et comp.) Ser. Mél. 1. p. 131.

9. Secale cereale. L.

A. Spica simplici. (mat.) Ser. Mél. 1. p. 135.

10. Hordeum hexastichon. L.

A. Spica hexasticha. (mat: et compr.) Ser.Mél. 1. p. 142 .

11. Hordeum vulgare. L.

A. Seminibus vestitis; spica flavescente. (mat. et compr.) Ser. Mél. 1. p. 145. 
12. HoRdEUM DISTICHON. L.

A. Seminibus vestitis; spica flexili, elongata; spiculis laxe imbricatis. (mat. et compr.) Ser. Mél. 1. p. 150.

B. Seminibus vestitis; spica rigida, brevi; spiculis dense imbricatis. (mat. et compr.) Ser. Mél. 1. p. 151.

C. Seminibus nudis, inflatis; spica flexili; spiculis laxe imbricatis. (mat. et compr.) Ser. Mél. 1. p. 152.

\section{HoRDEUM ZEOCRITON. L.}

(Mat. et compr.) Ser. Mél. 1. p. 153.

\section{Avena sativa. I.}

A. Glumella alba, aristata. (mat.) Ser. Mél. 1. p. 157.

B. Glumella alba, mutica. (mat.) Ser. Mél. 1.p. 159.

D. Glumella nigra, mutica. (mat.) Ser. Mél. 1.p. 160.

15. Avena orientalis. Schreg.

(Mat.) Ser. Mél.: 1. p. 162.

16. Avena fatua. L.

A. (Compr.) Ser. Mél. 1. p. 162 。

17. Phalaris canariensis L:

A. Spica magna. (mat. et compr.) Ser. Mél.1.p. 165. B. Spica exigua. (compr.) Ser. Mél. 1. p. 166. 


\section{Oriza sattva. L。}

(Compr.) Ser. Mél. 1. p. 168.

\section{Panicum mitiaceum. L.}

A. Panicula nutante; seminibus stramineis. (comp.) Ser. Mél. 1. p. 175.

B. Panicula erecta; seminibus badiis. (compr.) Ser. Mél. 1.p. 176.

\section{Panicum italicum. L.}

A. Spica elongata; seminibus stramineis. (mat.) Ser. Mél. 1. p. 17 T.

B. Spica subovoïdea; scminibus stramineis. (mat.) Ser. Mél. 1. p. 179.

C. Spica subovoïdea; seminibus aurantiacis. (mat.) Ser. Mél. 1. p. 179.

D. Spica subovoïdea; pedunculis violaceis. (mat.) Ser. Mél. 1. p. 180.

E. Spica exigua. (mat.) Ser. Mél. 1. p. 180.

\section{Mays vulgaris. Ser.}

A. Spica simplici; granis aurcis. (semina.) Ser. Mél. 1. p. 182.

B. Spica simplici: anis purpureis. (semina.) Ser. Mél. 1. p. 133.

C. Spica simplici; granis varicgatis. (semina.) Ser. Mí́l. 1. p. 184.

E. Caule humili; spica exili. (compr.) Ser. Mél. 1. p. 185. 
25. Cynosurus echinatus. L.

(Compr.) Ser. Mél. 1. p. 186.

27. Lolium temulentum. L.

(Compr.) Ser. Mél. 1. p. 18i.

28. Uredo carbo. DeC. (Brand.)

A. Hordei. (compr.) Ser. Mél. 1. p. 196.

C. Avenae. (compr.) Ser. Mél. 1. p. $19 \%$.

31. Urfido rubigo-vera. DeC. (host.)

A. Cerealis. (compr.) Ser. Mê 1. p. 202.

B. Agrostis. (compr.) Ser. Mél. 1. p. 202.

31. * Uredo caricina. DeC.

B. Caricis sylvaticae. (compr.) Ser. Mél.1. p. 202.

32. Sclerotium clavus DeC. (Mutterhorn.)

A. Cerealis. (compr.) Ser. Mél. 1. p. 203.

33. Fuccinil graminis. Pers.

A. (compr.) Ser. Mél. 1. p. 206.

B. (compr.) junior. Ser. Mél. 1. p. 20".

C. (compr.) senior. Ser. Mél. 1. p. 20־.

34. FA R I N.

A. Fleur de farinc. Ser. Mél. 1. p. 209.

B. Farine ordinaire. Ser. Mél. 1. p. 209. 
C. Farine de Maïs. Ser. Mél. 1. p. 210.

D. Farine de blé-corné. (T. durum.) Ser. Mél. 1. p. 210.

$$
\text { 35. } \mathrm{S} \circ \mathrm{N} \text {. }
$$

A. Gros-son. Ser. Mél. 1.p. 211.

B. Petit-son. Ser. Mél. 1. p. 212.

36. Gruiux DE bLÉS.

A. Gruau d'épeautre, semoule, simola. (Gries.) Ser. Mél. 1. p. 213.

B. Gruau d'épeautre colorié. Ser. Mél. 1. p. 213. C. Gruau de blé-corné. ('T. durum.) Simola de Gênes. Ser.Mél.1.p. 213.

37. Gruaux i'orges.

A. Gruau d'orge. 0. mondé. Ser. Mél. 1. p. 214. B. Gruau d'orge-perlé. O. d'Ulm. (Ulmergerste.) Ser. Mél. 1. p. 214.

38. Gruau de miliet.

Ser. Mél. 1. p. 215 :

39. Gruau d'avoine.

Ser. Mél. 1. p. 215.

40. Gruau d'avoine torréfié.

A. Haberkern. Ser. Mél. 1.p. 216.

B. Habermèl. Ser. Mél. 1. p. 216.

41. Pates d'Italie.

A. Vermicelle blanc. Scr. Mél. 1. p. 220. 
B. Vermicelle au safran. Ser. Mćl. 1. p. 220.

C. Lazaignes de Gènes. Ser. Mél. 1. p. 220.

D. Macaroni. Ser. Mél. 1. p. 221.

E. Pâtes en graines. Ser. Mél. 1. p. 221.

A. Tuyau de paille de blé blanchie. Ser. Mél. 1. p. 225.

B. Lanières de paille de blé, divisée par le fendoir. Ser. Mél. 1. p. 225.

C. Paiile de blé tissée. Ser. Mél. 1. p. 226.

D. Paille de blé cousue. Ser. Mél. 1. p. 226.

F. Paille de blé enduite de soufre. Ser. Mél. 1. p. 226.

F. Paille de riz. 227 . 


\section{TAELE DES NOMS LATINS}

de la monographie des céréales de la Suisse.

Nota. Les noms imprimés en caractères italiques sont ceux des genres et des espèces établis dans cette Monographie.

Agrostema gythago page 186. Anthera 79.

Avena 155.

alba 157.

fatua 162.

heteromalla 161.

nigra 160.

orientalis 160 .

racemosa 161.

sativa 157. A, $15 \%$. B. 159. C. 160. D. 160.

vulgaris 157.

Berberis vulgaris 194.

Calyx 70, 75.

Cariopsis 81.

Centaurea Cyanus 156.

Clavis siliginis 203.

Clavus 203.

Corolla 70, 76 .

Culmus 69,72 .

Cynosurus echinatus 186.

Filamentum 79.

Folium 69, 73.

Iructus 70,80 .

Frumenta 83.

Frumcutum indicum 152.

Gluna 70,75 .
G!umella, page 70, 78. Gramen avenaceum 162. Grana secalis degenerati 203. Halleri Histor. N. ${ }^{0}$

Secale 1421, 136.

Triticum. 1422 A. 93.

1422 B. 85.

entre 1422 et $1423.10 \%$. 1423. 111.

1424. 119, $125,131$.

Avena.

1494. 158.

1495. 163.

Hordeum.

1533. 146.

1534. 143.

1535. 151.

Hordea Pal. Beauv. 140.

Hordea distich. 139, 141. 149.

Hordea hexast. 139, 140, 142.

Hordeum 138, (Tableau 140.)

rlistichon 141, 149 A. 150.

B. 151. (Lin. 150.)

distichon nudum L. 153. distichum J. Bauh. 150. distichum imberbe 153. 
Hordeum.

Germanis oryza, p. 153. Hexastichon $140,142$. A. 142 . B. 144 . Hexastichon L. 142. nigrum Willd. 147. polystichum 145. polystichum vernum 145. Vulgare 141, 145. A. 145. B. 147. C. 148. vuigare L. 145 . vulgare nigrum 147. vulgare coleste 148. zeocriton 141, 153.

lepicena 75 .

Jocusta $69,7 \%$.

lodiculae 78.

Lolium perenne ramosum 96.

Lolium temulentum 187.

Lychnis gythago 187.

Lycoperdon tritici 197.

Mays quarantino 185.

Maysvulgaris 180. A.182. B. 183. C.184.D.184. E. 185.

Nectarium 70.

Oryza sativa $167,168$.

Panicula 6y, 74:

Panicum 172.

germanicum 177.

italicum 177. A. 17\%. B. 179. C. 179. D. 150 . E. 180 .

miliacerom 175.

milium 175.

vulgare 177 .

Wapaver Rhaeas 186.

Plialaris canariensis 164. A. 165. B. 166 .
Pistillum page 70,80 :

Polygonum fagopyrum 227. Puccinia graminis 188, 206.

A. 206. B. 207. C. 207.

Rachis 69, 74 .

Radix 69, 70.

Reticularia segetum $19 \%$.

Rosa (voyez table p. 57.)

sclerotium clavus 188, 203.

secale 135.

cereale 135, 136. A. 125.

B. 137 .

cereale compositum 137. cornutum 203.

hybernum 135.

luxurians 203.

spicis ramosis 137.

Secalis mater 203.

Speltce 83.

Sphaeria functiformis grami. naria $188,207$.

spica $69,73$.

spicula 99,74 .

squammae 70.

stamen 70,79 .

stragula 76.

tegmen 75.

Triticum 82, 53 .

xstivum L. 87, 90, 98, 99. amyleum 14. A. 125. B.

127. C, 123. D. 129.

E. 129.

amyleum atratum $\$ 2$.

atratum Host. 129.

Bauhini Lagasc.? 126. cienfuegos. Lagasc. 125. compactum arisatum 94. compactum snuticum 95 . 
Triticum.

compositum L. fil. p. 104. creticum 95.

dicoccum Fell. 126.

dicoccum Schrank. 119.

dicoccum persicum 126.

dicoccum romanum 126. dicoccuin russicum 128. durum $55,101,106$. A. 107. B. 108. C. 109. durum Tangarok 109. hordeiforme Host. 107, 109.

hybernum 90-94.

hybernum molle 92 .

Linnaeanum? 101.

monococcum L. 115. 131. monococcum majus 125. peruvianum 182 .

polonicum $85,100,110$, 111.

polonicum turg. Pers. 99. sardinicum 94. sativum $88,91,93$. sativum durum 108 . sativum, gris de souris, 103.

sativum, Pétaniel. blanc 98.

sativum, Pétaniel. roux 99.

sativum pyramid. 96, 101. sativum turgidum 101.

Spelta 66, 114, 116, 123. A. 118. B. 120. C. 120 . D. 120 .E. 121 . F. 122 . G. 122. H. 123, I. 123, 125.
Triticzm.

Spelta subcomposit. page 130.

tableau 84,114 .

'Tangarok. 109.

Touzelle Vill. 91.

turgidum 85, 97. A. 98. B. 98 . C. 101 . D. 102 .

E. 102. F. 103. G. 104. turgidum L. 99. Vili, 98. typhinum 109. venulosum 115, 133. villosum Host. 108. vulgare 84 ', 86. Vill. 88 , 91. A. 87. B. 89. C. 89 .

D. 90 . E. 90 . F. 92 . G. 93 . H. 94 . I. 94. J. 95 .

vulgare autumnal. 91,92 , 93.

vulgare hybernum 91. vulgare palmare 108. zea $117,119,126$. zea hybernum 125.

zea würtembergicum 126 . Uredo carbo 188, 195. avenae 197. hordei 196. tritici 197.

Caricina 202. Caries 188, 199. Maycadis 188 , 198. Rubigo-vera 188 , 201. Segetum avenae 197.

hordei 196.

Mays zeae 198.

tritici 197.

urceolorum 179. 
Ustilago page 196.

Zea amylea 127.

amylea seu olyra 125 .

amylea vel zeocriton 125 .

briza dicta 131.

Mays L. 182.

monococcos 131 .
Zea sive spelta page 1 is. spica mutica 121.

verna 125.

Zeocrita Pal. Beauv.141.

Zeocriton commune 154.

Zeocriton distichon 151, 152 .

TABLE DES NOMS FRANC,AIS.

Alpiste des canaries pag. 166. Anthère 79. Averon 164.

Avron 164. Avoine 155.

d'orient 163.

folette 164 .

ordinaire 159 .

ordinaire noire 160.

noire 160 .

unilatérale 162.

Baillard 151.

Bâle 70.

Barbot 186.

Blanchiment des pailles 225 . Blé 82.

amidonier 128.

amidonier à courtes barbes 128 .

amidonier noirâtre 129. amidonier rameux 130. amidonier velouté 128 . anglais 101. corné à deux couleurs 110.
Blé corné glabre paģe 109.

corné velouté 108.

d'abondance 98, 106.

de Bohême 92.

de Crête 97.

d'Egypte 111.

de Jérusalem 127.

de miracle 106.

de Mogador, 111.

de Pologne 111.

de Pologne serré 111.

de Sicile 101.

de Surinam 111.

divariqué 112.

grison 88.

Lammas 93.

Locar. 133.

Locular 133.

mottu 96, 97.

noir 227.

ordinaire 94.

trémois 88.

veiné 134.

Bleuet 186.

Bluet 186. 
Carabin page $22 \%$.

Carie 188 , 199.

Cariopse 81.

Céréales suisses 65 .

Chaume $69,72$.

Charbon 185, 195.

de l'avoine 197.

de l'orge 196.

du blé 197.

Civada cougucuida 164.

Coquelicot 186.

Corolle Michel. 78.

Cynosure hérissée 186.

Description des genres et espèces 82.

Distribution du mémoire 67.

Division des pailles 225.

Ecaille Lin. 78.

Epeautres 116.

barbu à épi blanc 119.

barbu, blanc et glabre 118, 119.

barbu, blanc et velouté 120.

barbu, roux et glab. 120 .

sans barbes, apauvri 123 .

sans barbes, blanc et gla.

bre 121 .

sans barbes, gris - bleu 123.

sans barbies, roux et gla. bre 122.

sans barbes, roux et ve-

louté 122.

sans barbes, violetet gla-

bre 122.

Exii $69,73$.

Epillet 69, 74 .
Ergat page 188, 203.

Ergotisme 205.

Escourgeon 144.

Etamine 70, 79.

Farines 209.

Fendoirs 225.

Feuille 69, 73 .

Filet 79.

Folle avoine $16 \%$.

Fruit $70,80$.

Froments 86, 114.

blanc de Montpellier 93 . commun 83.

à épi blanc 92 .

commun barbu à épi

compact. 97.

commun barbu, blanc et glabre 38 .

commun barbu, blanc et et velouté 88 .

commun barbu roux et glabre 90.

cominun barbu, roux et velouté 90.

commun sans barb. ̀̀ épi

blancet à graines jau-

nes 92 .

commun sans barb. blanc

et glabre 92 .

conmun sans barbes gla-

bee et roux 93 .

commun saus barb. ve-

louté et blanc 92 .

conmiun sans barb. ve-

louté et roux 94.

d'Alsace 97.

d'automne de épi blanc 88.

d'iutomne à épi doré 93.

Froment 
Froment de Barbarie, p.105. dur. 108.

grisâtre 92.

grisâtre épi velouté, graines dorées 92 .

gris-de-souris 103.

monocoque 133.

noir de Moutpell. 133.

rentlé 101.

rouge de Montpell. 99, $101,102$.

sans. barb. à épi doré 93. sans barb. à épi court 97. sans barb. d'Alsace 97.

uniloculaire 133.

Glume $70,75$.

calicinale 75 .

corolline 76 .

extérieure 75 . intérieure 76 .

Glumelle $70,76,78$.

Grande épeautre 127.

Gros-blé 101.

à épi roux et glabre 102.

à épi noir 103.

à épi noir et compact 102.

à épi rameux 106. mutique 104 .

noir à épillets écart. 103. sans barbes 104.

Gruatux 212.

d'avoine 215.

d'avoine torréfié 216.

de blé-corné 203.

de millet 215 .

d'épeautre 213.

d'orge 214.

d'orge perlé 214.
Introduction à l'étude dés céréales ; page 69.

Ivraie 187.

Lazaignes de Gênes 220.

Lépicène 75 .

Lodicules 78.

Macaroni 221.

Mlä̈s 181.

à grains dorés 153.

à gr. panachés 184 .

à gr. pourprés $1 \mathrm{S3}$.

rameux 184.

Maỉadies des céréales 187.

Mil. 176. Millet 172.

à graines brunes 177 .

à gr. jaunes 176 .

à gr. oranges 150 .

apauvri 180.

des oiseaux à épi along. 179.

des oiseaux à épi ovoïde page 179.

Moutin blanc 98.

Nectaire 70.

Nectaire Schreb. 78.

Nielle 186.

Niclle (malad.) 1s8, 195.

Ordre des citations 87.

Organes de la reproduction

$71,73,75,81$.

Organes de la végétation $69-75$.

Orge 138.

à café 152 .

à deux rangs 151 .

a deux rangs nue 152 .

anguleuse 144 .

à six côtés 144 . 
Orge à six rangs page 144 . carrée 144.

commune 147. commune à gr. nues 149 . de Jérusalem 149.

de Russie 154. de Sibérie 149.

d'Espagne 152.

d'hiver 144.

distique 152.

dist. à épill. rapproch. 151.

dist, sans barb. 153.

d'UIIm 214.

du Pérou 152.

en éventail 154 .

faux riz 154.

mondé 214.

perlé 214 .

pyramidal 154.

Orges distiques 149.

Orges hexastiques, p. 139.

Osties 221.

Oublies 221.

Pailles 222.

Pains à cacheter 221.

Pamelle 151.

Panicule 69, 74.

Paoumoule 151.

Pàtes de Gênes 219.

de graines 221.

d'Italie 219.

Périgone $70,76$.

Pétanielle blanc 98 .

blanche 98 .

noire $102,103$.

rouge 101 .

rousse $98,99,101$,

Pelit épeautre 133.
Pistil, page $70,80$.

Ponceau 156.

Poulenta 182.

Poulinte 182.

Rachis 69-74.

Racine 69-i0.

Rafle 69,74 .

Riz 167 .

d'Allemagne 154 ,

rustique 154 .

Rizières 168.

Rouille 188, 201.

Saisette 88.

de Tarascon 90.

Sarazin 227.

Seigle 135.

commun 137.

commun rameux 239 .

de printemps 136.

Semoule 213.

Simola de Gênes, 213.

de Suisse 213.

Son 211.

Soucrion 144.

Stragule 76 .

Tangarok 109, 110.

'Tissage des pailles 225.

Touzelle 57.

blanclie harbue 90.

blanche sans barb. 91,92 ,

rouge barbue 90 .

rouge sans barbes 93.

Touz lles du nidi compa-

rées av. le T.vulgare 87.

Urédo du Maïs 185, 195.

Usages economiques des

céréales $20 \mathrm{~s}$.

Vermicelle blanc 220.

Vermicelle au safran 220 . 


\section{TABLE DES NOMS ALLEMANDS.}

Acgyptischer Winterweizen page 128.

Aehrchen 69.

Aehre 69.

Amer 127.

Aemer 127.

Aemerkorn 127.

Bartgerste 154.

Betïubender Lolch 157.

Binkelweizen 94, 97.

Blatt 69.

Blicken 133.

Böhmisch. Sammtweizen 93.

Brand 188, 195.

Canarien_Gras 166.

Canarien-Saamen 166.

Corallenweizen 119.

Cretischer Weizen 97.

Denkel 119.

Dinkelkorn 119.

Dinkelweizen 119.

Dünkel 119.

Einkorn 133.

Einkörniger Weizen 133 ,

Englischer Weizen 101.

Fadennudeln 220.

Flughaber 164.

Frucht 70 .

Gemeiner Haber 159.

Gemeiner Mays 183.

Gemeiner Roggen 137.

Gemeiner Weizen 89.

Graupen 214.

Gries 213.

Haberkern_216.
Habermehl page 216.

Halm 69.

Hirsenfennich 176 .

Hirss 176.

Immer 127.

Jerusalemkorn $12 \%$.

Kalg 70.

Kälglein 70 .

Kerngerste 147.

Korn 119.

Kornfäule 198, 199.

Kornfrass 1S8, 199.

Mutterkorn 188, 203.

Nackte Gerste 152.

Perlgerste 214.

Polnische zweyzeilige Sommergerste 152.

Polnischer Weizen 111.

Rispe 69.

Rollgerste 144 .

Romanischer Sommerwei zen 127.

Rost $18 \mathrm{~s}$.

Russischer Mehldinkel 128.

Sankt_Peterskorn 133.

Sechszeilige Gerste 144.

Sommerweizen aus Esula 97.

Spelze 119.

Spelt 119.

Spindel 69.

Staubgefäss 70 .

Staubweg 70.

Stockgerste 144.

Türkischer Hafer 162.

Ulmergerste 214. 
Vielkörniger Weizen, page 106.

Wallachisches Korn 111.

Welscher Fennich 179.

Wilder Hafer 164.
Wintergerste page 147 .

Winterweizen $92,93$.

Wurzel 69.

Zweykorn 119.

Zweyzeilige Gerste 151.

\section{TABLE DES NOMS ANGLAIS.}

Battle_door-barbey 155.

Beardel Oat-grass 164.

Beardet wild Oats 164.

Canary-Grass 166. ..

Fuiham-barbey 155.

Myle 176.
Parney-barbey 155. Sommer-wheat 89. Sprat_barbey 155. Weit-Spring-wheat 89 . Wheat 89.

\section{TABLE DES NOMS ESPAGNOLS,}

Carraon 133.

Escanna 133.

Esprilla 133.

Milhe 176.
Redondillo 101.

Trigo-de-Polonia 111. Trigo Moruno 108.

TABLE DES NOMS ARABES.

Dourach châmy 183.

Durach kyrân 183.

Qamh a'raby 101.
Qamh meyhayz 101. Qainh neygeh 101. T'ourky 183.

TABLE DES NOMS ITALIENS.

Granosprone 203.

Macaroni 220.

Orzo die germania 155.

Simola 213.

Vernicellis 220.

TABLE DES NOMS SUEDDOIS.

Canarie Frö 166.

Skyfel korn. 155.

Plumage lkorn 155. 

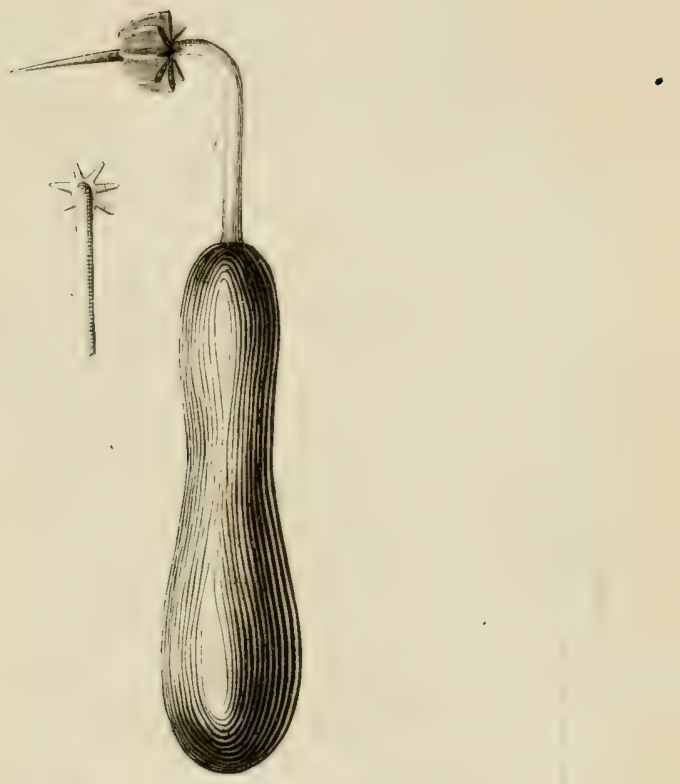




\section{TABIEAU METHODIQUE}

\section{DES CÉRÉALES,}

\section{DÉCRITES DANS CETTE MONOGRAPHIE.}

Nota. Les Nros., qui précédent chaque espéce dans ce tableau, sont les mêmes que ceux qui sont placés dans le corps de l'ouvrage devant ces-mêmes especes.

\section{TrITICUM.}

Gluma bivalvi, quadriflora; seminibus obtusis, pag. $\$ 2$.

\section{SE C A L E.}

Gluma bivalvi, biflora; seminibus truncatis

H O R D EUM.
bivalvi, unifora; spi-
rnis in verticillum di-
$m$ congestis, pag. 138 .

Hexasticha.

Gluma bivalvi, uniflora; spiculis ternis in verticillum dimidiatum congestis, pag. 138.

\section{A VENA.}

Gluma bivalvi, bi seu $3-5$ flora; valvula exteriori glumellae dorso aristata; p. 155.

\section{PHA LA R IS.}

Glumella bivalvi, unifora; carina alata.

$$
0 \text { R Y Z A. }
$$

Gluma bivalvi, unifora; glumella rugoso-punctata.

$$
\text { PA N I C U M. }
$$

Gluma trivalvi, unifora, p. 172.
1. Vulgare. Seminibus ovoildeis, obtusis, p. 86. (10 var.)

2. Turginum. Seminibus ovoïdeis, gibbis, p. 97. (7 var.)

3. Durum. Seminibus ellipsoïdeis, gibbis, p. 106. (3 var.)

4. Polonicum. Seminibus ellipsoïdeis, subtriquetris, p. 110. (2 var.)

5. Speuta. Seminibus triquetris, p. 116, (9 var:)

6. AmYleum. Seminibus triquetris, gibbis, p. 124. (5 var.)

7. Movococcum* Seminibus compresso-triquetris, p. 130. (1. var,)

8. Ventoosum. Carina venulosa, p. 133. (1. var.)

9. Cereale. p. 135. (2 var,

10. HexastichoN. Spica rigida, ascendente, p. 142. (2 var.)

11. Vulgare. Spica flexili, nutante, p. 145. (3 var.)

. $\quad$ 12. Distichon. Spica compressa, lateribus parallelis, p. 149. (4. var)

13. Zeocriron. Spica ‘ impressa, pyramidali, p. 153. (1 var.)

14. Sativa, Panicula के uali, p. 157. (4 var.)

15. Orrentalis. Panic contracta, p. 160. (1 var.)

16. Fatua. Giuma pile 1, p. 162. (1 var.)

17. Canariensis. p. $16 \mid(2$ var. $)$

18. S.ativa. p. 167. (1 vi )

19. Milraceum, Floribus paniculatis, p. 175. (2 var.)

20. Italicum. Floribus spicatis, p. 177. (5 var.)

\section{A $\mathbf{Y} \mathbf{s}$.}

Floribus monoicis; masculis paniculam terminalem constituentibus.

21. VuLGaris. p, 1SO. (5. var.) 




\section{OK3 New York Botanicai Garden Library \\ QK3.54 v.1 pt.1 - 2 \\ Seringe, Nicolas $\mathrm{Ch} /$ Melanges botaniques,

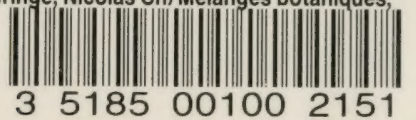




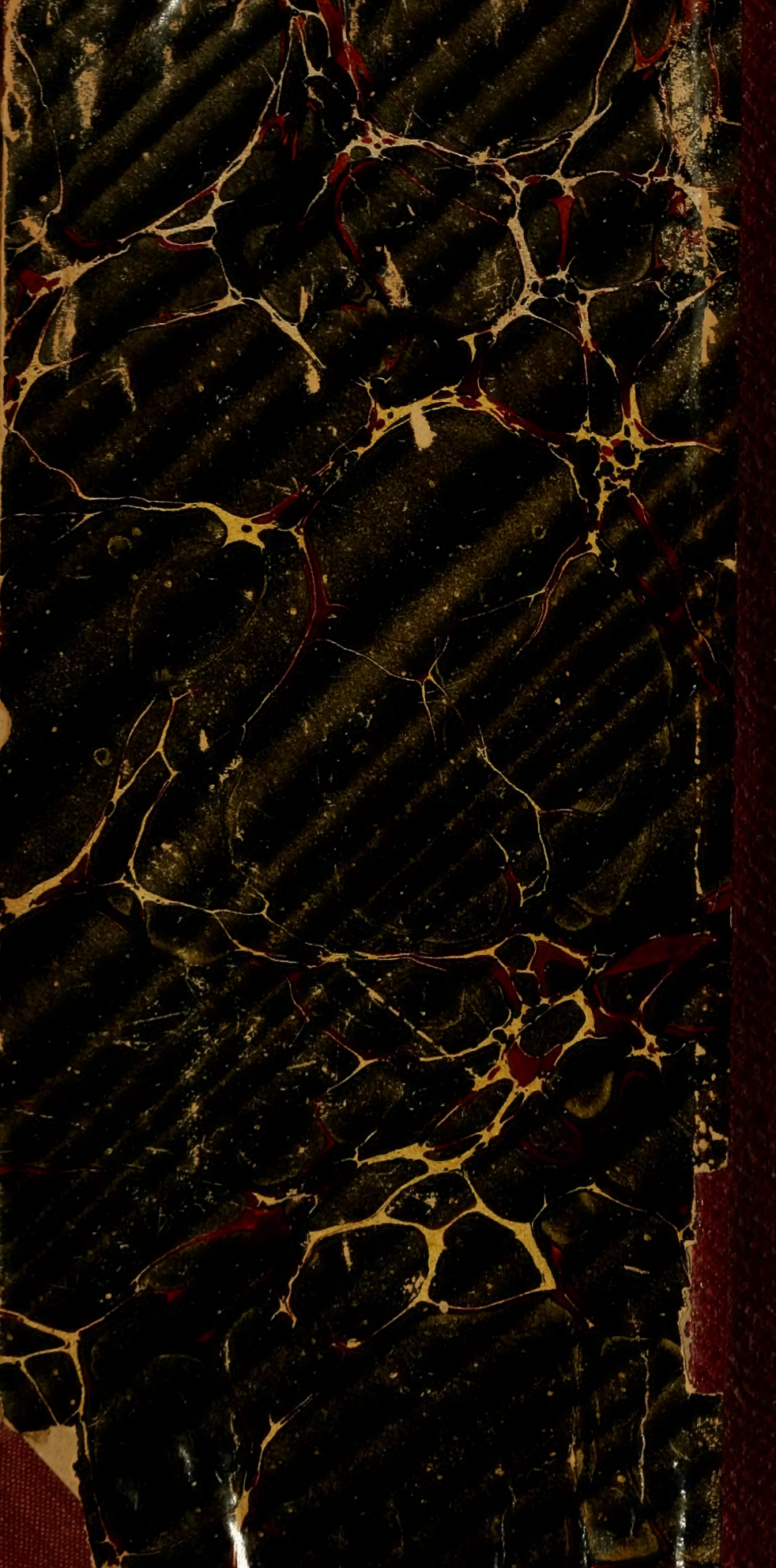

